\begin{tabular}{|c|l|}
\hline Title & Higher dimensional SLEP equation and applications to morphological stability in Polymer Problems \\
\hline Author(s) & Nishiura, Y asumasa; Suzuki, Hiromasa \\
\hline Citation & $\begin{array}{l}\text { SIAM Journal on Mathematical A nalysis, 36/3), 916-966 } \\
\text { https://doi.org/10.1137/S0036141002420157 }\end{array}$ \\
\hline Issue Date & 2005 \\
\hline Doc URL & http://hdl.handle.net/2115/35224 \\
\hline Rights & ○ 2004 Society for Industrial and A pplied Mathematics \\
\hline Type & article \\
\hline File Information & nishiura 10.pdf \\
\hline
\end{tabular}

Instructions for use 


\title{
HIGHER DIMENSIONAL SLEP EQUATION AND APPLICATIONS TO MORPHOLOGICAL STABILITY IN POLYMER PROBLEMS*
}

\author{
YASUMASA NISHIURA ${ }^{\dagger}$ AND HIROMASA SUZUKI ${ }^{\ddagger}$ \\ Dedicated to Professors Masayasu Mimura and Takaaki Nishida \\ on their sixtieth birthdays
}

\begin{abstract}
Existence and stability of stationary internal layered solutions to a rescaled diblock copolymer equation are studied in higher dimensional space. Rescaling is necessary since the characteristic domain size of any stable pattern eventually vanishes in an appropriate singular limit. A general sufficient condition for the existence of singularly perturbed solutions and the associated stability criterion are given in the form of linear operators acting only on the limiting location of the interface. Applying the results to radially symmetric and planar patterns, we can show, for instance, stability of radially symmetric patterns when one of the components of diblock copolymer dominates the other, and that of the long-striped pattern in a long and narrow domain for the planar case. These results are consistent with the experimental ones. The above existence and stability criterion can be easily extended to a class of reaction diffusion systems of activator-inhibitor type.
\end{abstract}

Key words. singular perturbation, diblock copolymer, reaction diffusion system, pattern formation, matched asymptotic expansion, stability, critical eigenvalues

AMS subject classification. 35B25, 35B35, 35K57

DOI. $10.1137 /$ S0036141002420157

1. Introduction. In this paper, we shall discuss two basic problems of singularly perturbed solutions to the fourth order equation (1.1) arising in modelling the dynamics of diblock copolymer melts in higher dimensional space, namely Under what conditions existence of stationary singularly perturbed solution is guaranteed? and How is the stability of it? The former is related to the possible morphological forms, and the latter is crucial for observability. We shall see that both problems are eventually reduced to solving the equations on the interface in the singular limit. Especially, the spectral problem on the interface called the SLEP equation in higher dimensional space (see (1.13) and (4.22)), is a generalization of a one-dimensional version for reaction diffusion systems discussed in [11] and [10]. The model equation (1.1) below looks very special; however, the method employed here is quite general and can be extended to the activator-inhibitor systems treated in [11] and [10], in fact the mechanisms causing pattern formation have common features in both models. Our model takes the following form:

*Received by the editors December 27, 2002; accepted for publication (in revised form) December 12, 2003; published electronically November 17, 2004. This research was partially supported by the Grant-in-Aid for Scientific Research 11740060, 12440026, 13440027, the Japanese Society of the Promotion of Science.

http://www.siam.org/journals/sima/36-3/42015.html

${ }^{\dagger}$ Laboratory of Nonlinear Studies and Computations, Research Institute for Electronic Science, Hokkaido University, Kita-ku, Sapporo, 060-0812, Japan (nishiura@aurora.es.hokudai.ac.jp).

${ }^{\ddagger}$ Faculty of Education, Shiga University, Hiratsu, Otsu, 520-0862, Japan (kodachi@sue. shiga-u.ac.jp). 


$$
\left\{\begin{array}{ll}
u_{t}=-\Delta\left\{d^{2} \Delta u+f(u)-\sigma\left(-\Delta_{N}\right)^{-1}(u-\bar{u})\right\} & \\
& =-\Delta\left\{d^{2} \Delta u+f(u)\right\}-\sigma(u-\bar{u}),
\end{array} \quad(X, t) \in \hat{\Omega} \times(0, \infty),\right.
$$

where $u$ is the order parameter which represents the ratio of two homopolymers, $f$ is basically a bistable nonlinearity (typically $u-u^{3}$ ), $d$ and $\sigma$ are positive constants, $\Delta_{N}$ is the Laplace operator under the Neumann boundary condition, and $\hat{\Omega}$ is a smooth bounded domain in $\mathbf{R}^{N}(N \geq 2)$. Originally, the model system was given by the energy functional form in [16] (see also [1]), and then reformulated in the above form by [12], which triggered many interesting rigorous works such as [25] and [26]. The associated Euler-Lagrange equation with the functional is given by (1.1). Diblock copolymer is a chain where two different homopolymers are connected, and the connectivity causes a long range interaction. In fact, its effect is reflected by the nonlocal term $\sigma\left(-\Delta_{N}\right)^{-1}(u-\bar{u})$, where $\sigma$ is proportional to the inverse of the polymerization index (i.e., length of the chain). Due to the nonlocal term $\sigma\left(-\Delta_{N}\right)^{-1}(u-\bar{u})$, (1.1) displays a variety of stationary mesoscopic patterns including lamellar, column, spherical, double gyroid morphologies and so on (see, for instance, [24] and the reference therein). Here the mesoscopic means an intermediate scale between micro and macro, and it becomes very fine as $d$ tends to zero as in the following proposition. This makes a sharp contrast with the case $\sigma \equiv 0$, i.e., Cahn-Hilliard equation. For experimental observation, see $[6,7,8]$. Note also that the nonlocal effect is similar to the role of the inhibitor field for activator-inhibitor system, and hence our analysis basically encompasses such a system as we shall see in section 5 .

In order to have a reasonable singular limit, some sort of scaling law is necessary and we adopt the following one, which was originally proposed in [12] in the above setting and was proved rigorously by [14] for one-dimensional case and by [2] for higher dimensional case.

Proposition 1.1. (Theorem 3.2 in [12]). In order to have a well-defined limiting stationary problem of (1.1) as $d \downarrow 0$, which is independent of parameters $d$ and $\sigma$, there exists a unique scaling with respect to space and time given by: $x:=X /\left(d^{1 / 3} \sigma^{-1 / 3}\right)$, $\tau:=\sigma t$. The characteristic domain size is proportional to $(d / \sigma)^{1 / 3}$ and the morphology of pattern is determined by solving (1.7)-(1.9) (see assumption (A4)).

This scaling law is not only a sufficient condition in order to have a well-defined singular limit problem of (1.1) as $d \downarrow 0$ (see [12] for details), but also can be justified from a view point of statistical physics (see [3]). In terms of the new variables $x$ and $\tau$, the equations in (1.1) are recast as

$$
u_{\tau}=\Delta\left\{-\epsilon \Delta u-\frac{1}{\epsilon} f(u)\right\}-(u-\bar{u}), \quad(x, \tau) \in \Omega \times(0, \infty)
$$

where $\epsilon:=d^{2 / 3} \sigma^{1 / 3}$ and $\Omega$ is a magnified unit domain. Here we implicitly assume that the patterns are periodic in space (in the original scaling), and we focus on a unit cell of this periodic structure. 
On the other hand, a typical activator-inhibitor system is given by

$$
\left\{\begin{array}{l}
u_{t}=d^{2} \Delta u+f(u)-v, \\
v_{t}=D \Delta v+g(u, v),
\end{array} \quad(x, t) \in \hat{\Omega} \times(0, \infty),\right.
$$

where typically $(f, g)=\left(u-u^{3}, u-\gamma v\right)(\gamma>0)$. It was proved in [13] that if (1.3) has a $d$-family of stationary matched asymptotic solutions whose interface is smooth up to $d=0$, then it must become unstable for small $d$. It is observed numerically that stable patterns become finer and finer when $d$ becomes small (see also a recent work [15]), which strongly suggests the necessity for rescaling in order to track the stable patterns. It was also shown in [13] that the characteristic size of stable patterns is of order $d^{1 / 3}$ by formal computation. Magnifying the system (1.3) with this scaling, the rescaled system is given by

$$
\left\{\begin{array}{l}
u_{t}=\epsilon^{2} \Delta u+f(u)-v, \\
\epsilon v_{t}=D \Delta v+\epsilon g(u, v),
\end{array} \quad(x, t) \in \Omega \times(0, \infty),\right.
$$

where $\epsilon=d^{2 / 3}$. (See $[4,18,19,20,21,23]$.)

At first sight there is no resemblance between (1.2) and (1.4); however, rewriting (1.2) by introducing new variable $v:=\epsilon^{2} \Delta u+f(u)$ and new time $\tilde{t}:=\tau / \epsilon$, the resulting system becomes

$$
\begin{gathered}
\left\{\begin{array}{l}
0=\epsilon^{2} \Delta u+f(u)-v, \\
0=\Delta v+\epsilon(u-\bar{u})+u_{\tilde{t}},
\end{array} \quad(x, \tilde{t}) \in \Omega \times(0, \infty),\right. \\
\frac{\partial u}{\partial n}=0=\frac{\partial v}{\partial n}, \quad(x, \tilde{t}) \in \partial \Omega \times(0, \infty) .
\end{gathered}
$$

Now it is clear that (1.2) is similar to (1.4) as far as stationary solutions are concerned. Similarity in fact can be extended to dynamical level (i.e., stability), which will be discussed in section 5 .

As a consequence of the above discussion, (1.5) is expected to have a well-defined smooth interface in the singular limit. The next issue is to locate such an interface by solving, what is called, the reduced problem of (1.5) (see (1.7)-(1.9)); however, it is in general quite difficult to solve (1.7)-(1.9) and obtain its explicit profile. The results listed below will be, therefore, stated under the assumption that such reduced solutions exist. Nevertheless, this assumption can be checked explicitly for simple but basic cases when $\Omega$ has a spherical or planar geometry, which will be shown in later sections.

Now we shall state the assumptions and introduce several key notation and operators for later use.

(A1) $f$ is a smooth bistable nonlinearity. The equation $f(u)-v=0$ has three sub-branches of solutions

$$
\begin{aligned}
& \mathcal{C}_{+}=\left\{(u, v) \mid u=h^{+}(v), v \in I_{+}\right\}, \mathcal{C}_{-}=\left\{(u, v) \mid u=h^{-}(v), v \in I_{-}\right\} \quad \text { and } \\
& \mathcal{C}_{0}=\left\{(u, v) \mid u=h^{0}(v), v \in I_{+} \cap I_{-}\right\}
\end{aligned}
$$

such that $h^{-}(v)<h^{0}(v)<h^{+}(v)$ for $v \in I_{+} \cap I_{-}$. 
(A2) $f_{u}\left(h^{ \pm}(v)\right)=\frac{d}{d u} f\left(h^{ \pm}(v)\right)<0$ on $I_{ \pm}$.

(A3) $J(v):=\int_{h^{-}(v)}^{h^{+}(v)}[f(s)-v] d s, v \in I_{0}$, has an isolated zero $v^{*} \in I_{0}$ such that $J^{\prime}\left(v^{*}\right)<0$.

(A1)-(A3) with (A4') and (A7) assumed later are the sufficient conditions for the existence and stability of transition layer solutions to one-dimensional activator-inhibitor system (1.3) (see [10] and [11]).

We assume that the domain $\Omega$ is simply connected, and $\Gamma$ is either one of the following two cases.

Case I. $\Omega \in \mathbf{R}^{N}(N \geq 2)$ has smooth boundary and define a set $\mathcal{F}$ by

$$
\begin{aligned}
\mathcal{F}=\{\Gamma \subset \Omega \mid \Gamma \text { is an } N-1 \text { dimensional smooth compact } \\
\text { connected manifold without boundary }\}
\end{aligned}
$$

Each $\Gamma \in \mathcal{F}$ divides $\Omega$ into two connected components. We denote by $\Omega_{+}(\Gamma)$ the component of $\Omega \backslash \Gamma$ which has $\partial \Omega$ as part of its boundary. The other component is defined by $\Omega_{-}(\Gamma)$. Therefore we have

$$
\partial \Omega_{+}(\Gamma)=\partial \Omega \cup \Gamma \quad \text { and } \quad \partial \Omega_{-}(\Gamma)=\Gamma .
$$

Case II. $\Omega \in \mathbf{R}^{2}$ is a rectangle $(0, X) \times(0, Y)$. Then we define $\mathcal{F}$ by

$$
\begin{aligned}
\mathcal{F}=\{ & \{\Gamma \subset \Omega \mid \Gamma \text { is a line, which is parallel to the } y \text {-axis } \\
& \text { and touches transversely with the boundary } \partial \Omega\} .
\end{aligned}
$$

Then $\Omega$ is divided into two connected components $\Omega_{-}(\Gamma)=\left(0, x_{0}\right) \times(0, Y)$ and $\Omega_{+}(\Gamma)=\left(x_{0}, X\right) \times(0, Y)$, where $\Gamma=\left\{\left(x_{0}, y\right) \in \mathbf{R}^{2} \mid 0 \leq y \leq Y\right\}$ for some $x_{0} \in(0, X)$. For later use we introduce the aspect ratio $\kappa$ of $\Omega$, i.e., $\kappa:=X / Y$.

Let us introduce a local coordinate system in the neighborhood of $\Gamma$. By the implicit function theorem there exists a $d_{0}>0$ such that the map $p:\left[-d_{0}, d_{0}\right] \times \Gamma \rightarrow$ $\Gamma_{d_{0}}$ defined by

$$
p(r, y)=y+r \nu(y)
$$

is a diffeomorphism, where $\Gamma_{d_{0}}=\left\{x \in \Omega \mid \operatorname{dist}(x, \Gamma)<d_{0}\right\}, \nu=\nu(y)$ is the unit normal vector on $\Gamma(y \in \Gamma)$ which points the interior of $\Omega_{+}(\Gamma)$. Using this diffeomorphism, we identify $x \in \Gamma_{d_{0}}$ with $(r, y) \in\left[-d_{0}, d_{0}\right] \times \Gamma_{d_{0}}$ and write $u(r, y)$ or $u(y+r \nu(y))$ for $u(x)\left(x \in \Gamma_{d_{0}}\right)$. We denote by $H(r, y)$ the mean curvature of the manifold

$$
\Gamma(r):=\{x \in \Omega \mid x=y+r \nu(y) y \in \Gamma\}
$$

at $y \in \Gamma$. Then $H(0, y)$ stands for the mean curvature of the manifold $\Gamma$ at $y \in \Gamma$.

Such an interface $\Gamma \in \mathcal{F}$ is determined by solving the following equations called the rescaled reduced problem:

$$
\begin{aligned}
& \begin{cases}\Delta V^{-}+h^{-}\left(v^{*}\right)-\bar{u}=0 & \text { in } \Omega_{-}(\Gamma), \\
V^{-}=b_{1}^{*}(y) & \text { on } \Gamma,\end{cases} \\
& \begin{cases}\Delta V^{+}+h^{+}\left(v^{*}\right)-\bar{u}=0 & \text { in } \Omega_{+}(\Gamma), \\
V^{+}=b_{1}^{*}(y) & \text { on } \Gamma, \\
\frac{\partial V^{+}}{\partial n}=0 & \text { on } \partial \Omega,\end{cases}
\end{aligned}
$$




$$
\frac{\partial V^{+}}{\partial \nu}=\frac{\partial V^{-}}{\partial \nu} \quad \text { on } \Gamma
$$

where

$$
b_{1}^{*}(y):=\frac{m^{2}}{[h]}(N-1) H(0, y), m^{2}:=\int_{-\infty}^{\infty}\left[u_{\xi}^{*}(\xi)\right]^{2} d \xi
$$

and $[h]$ denotes the jump of two branches $u=h^{ \pm}(v)$ at $v=v^{*}$, i.e.,

$$
[h]:=h^{+}\left(v^{*}\right)-h^{-}\left(v^{*}\right) .
$$

Here $u=u^{*}(\xi)$ is the unique solution of

$$
u_{\xi \xi}+f(u)-v^{*}=0, \quad \xi \in \mathbf{R}, u(0)=h^{0}\left(v^{*}\right), \lim _{\xi \rightarrow \pm \infty} u(\xi)=h^{ \pm}\left(v^{*}\right) .
$$

When $\Gamma$ is a rectangle, we add the boundary condition

$$
\frac{\partial V^{-}}{\partial n}=0 \quad \text { on } \partial \Omega \cap \partial \Omega_{-}(\Gamma)
$$

to (1.7) and replace the boundary condition for $\frac{\partial V^{+}}{\partial n}$ in (1.8) by

$$
\frac{\partial V^{+}}{\partial n}=0 \quad \text { on } \partial \Omega \cap \partial \Omega_{+}(\Gamma) .
$$

Roughly speaking, the interface $\Gamma$ is a set on which the limiting function of the stationary solution $u^{\epsilon}$ as $\epsilon \downarrow 0$ becomes discontinuous in the normal direction. For more discussions on (1.7)-(1.9), see [12]. We assume the existence of a solution $\left(V^{*}, \Gamma^{*}\right)$ of $(1.7)-(1.9)$.

(A4) There exists a solution $\left(V^{*}, \Gamma^{*}\right) \in C^{1}(\bar{\Omega}) \times \mathcal{F}$ of $(1.7)-(1.9)$, where

$$
V^{*}(x)= \begin{cases}V^{-}(x), & x \in \Omega_{-}\left(\Gamma^{*}\right), \\ V^{+}(x), & x \in \Omega_{+}\left(\Gamma^{*}\right) .\end{cases}
$$

$V^{ \pm}$are smooth solutions of (1.7) and (1.8), respectively, satisfying (1.9).

In order to state a sufficient condition for the existence and the SLEP equation, we need to define several operators. Let us consider the following linear boundary value problem:

$$
\begin{gathered}
\begin{cases}\Delta Z^{-}=0 & \text { in } \Omega_{-}\left(\Gamma^{*}\right), \\
Z^{-}=q & \text { on } \Gamma^{*},\end{cases} \\
\left\{\begin{array}{l}
\Delta Z^{+}=0 \text { in } \Omega_{+}\left(\Gamma^{*}\right), \\
\frac{\partial Z^{+}}{\partial n}=0 \text { on } \partial \Omega, Z^{+}=q \text { on } \Gamma^{*},
\end{array}\right.
\end{gathered}
$$

where $\Gamma^{*} \in \mathcal{F}$ is a solution of (1.7)-(1.9). When $\Gamma^{*}$ is a rectangle, we add the boundary condition

$$
\frac{\partial Z^{-}}{\partial n}=0 \quad \text { on } \partial \Omega \cap \partial \Omega_{-}\left(\Gamma^{*}\right)
$$


to (1.10) and replace the boundary condition for $\frac{\partial Z^{+}}{\partial n}$ in (1.11) by

$$
\frac{\partial Z^{+}}{\partial n}=0 \quad \text { on } \partial \Omega \cap \partial \Omega_{+}\left(\Gamma^{*}\right)
$$

For $q \in C^{2, \alpha}\left(\Gamma^{*}\right)(0<\alpha<1)$, the problem (1.10) and (1.11) have a unique solution denoted by $\mathcal{P}^{-} q$ and $\mathcal{P}^{+} q$, respectively, satisfying $\mathcal{P}^{ \pm} q \in C^{2, \alpha}(\bar{\Omega})$, and define normal derivative operator $\Pi_{ \pm}: C^{2, \alpha}\left(\Gamma^{*}\right) \rightarrow C^{1, \alpha}\left(\Gamma^{*}\right)$ by

$$
\Pi_{-} q=\left.\frac{\partial}{\partial \nu}\left(\mathcal{P}^{-} q\right)\right|_{\Gamma^{*}}, \Pi_{+} q=-\left.\frac{\partial}{\partial \nu}\left(\mathcal{P}^{+} q\right)\right|_{\Gamma^{*}} .
$$

Note that $\Pi:=\Pi_{-}+\Pi_{+}$is self-adjoint and the null space $\mathcal{N}(\Pi)$ is one-dimensional spanned by a constant function. Then there is a bounded operator

$$
\mathcal{T}:(I-P) C^{1, \alpha}\left(\Gamma^{*}\right) \rightarrow(I-P) C^{2, \alpha}\left(\Gamma^{*}\right)
$$

satisfying $\Pi \mathcal{T}=I$ on $(I-P) C^{1, \alpha}\left(\Gamma^{*}\right)$ and $\mathcal{T} \Pi=I-P$ on $C^{2, \alpha}\left(\Gamma^{*}\right)$, where $P$ is a projection defined by $P \theta:=\frac{1}{\left|\Gamma^{*}\right|} \int_{\Gamma^{*}} \theta d S$ for $\theta \in C^{\alpha}\left(\Gamma^{*}\right)$ and $\left|\Gamma^{*}\right|$ is surface area of $\Gamma^{*}$.

Concerning the existence of the stationary solution, we have the following theorem.

Theorem 1.2 (existence). Assume that (A1)-(A4) are satisfied. Moreover, we assume that

(A5) There is a bounded linear operator $L^{\dagger}:(I-P) C^{\alpha}\left(\Gamma^{*}\right) \rightarrow(I-P) C^{2, \alpha}\left(\Gamma^{*}\right)$ which is the inverse of the operator

$$
L:=\Delta^{\Gamma^{*}}+H^{*}(y)-\frac{1}{m^{2}} J^{\prime}\left(v^{*}\right) V_{r}^{*}(0, y)+\frac{1}{m^{2}}[h] J^{\prime}\left(v^{*}\right) \mathcal{T}(\cdot)
$$

such that $L L^{\dagger}=I$ on $(I-P) C^{\alpha}\left(\Gamma^{*}\right)$ and $L^{\dagger} L=I-P$ on $(I-P) C^{2, \alpha}\left(\Gamma^{*}\right)$. Here, $\Delta^{\Gamma^{*}}$ is the Laplace-Beltrami operator on $\Gamma^{*}, H^{*}(y)$ the sum of the square of the principal curvature of $\Gamma^{*}, V_{r}^{*}(0, y)=\frac{\partial V^{*}}{\partial \nu}$, and $[h]$ denotes the aforementioned jump at $v=v^{*}$.

Then, there is an $\epsilon_{0}>0$ such that (1.5)-(1.6) have an $\epsilon$-family of stationary solutions $\left(u^{\epsilon}, v^{\epsilon}\right)$ for $\epsilon \in\left(0, \epsilon_{0}\right]$ satisfying

(i) $\lim _{\epsilon \rightarrow 0} v^{\epsilon}(x)=v^{*}$ uniformly on $\bar{\Omega}$,

(ii) for each $\delta>0$,

$$
\lim _{\epsilon \rightarrow 0} u^{\epsilon}(x)=\left\{\begin{array}{ll}
h^{-}\left(v^{*}\right), & x \in \bar{\Omega}_{-}\left(\Gamma^{*}\right) \backslash \Gamma_{\delta}^{*} \\
h^{+}\left(v^{*}\right), & x \in \bar{\Omega}_{+}\left(\Gamma^{*}\right) \backslash \Gamma_{\delta}^{*}
\end{array} \quad\right. \text { uniformly, }
$$

where $\Gamma_{\delta}^{*}$ is a tubular neighborhood of $\Gamma^{*}$,

(iii) for each $K>0$,

$$
\lim _{\epsilon \rightarrow 0} u^{\epsilon}(y+\epsilon \xi \nu(y))=u^{*}\left(\xi+s^{*}(y)\right) \quad \text { in } C^{2}[-K, K]
$$

uniformly in $y \in \Gamma^{*}$ for some $s^{*} \in C^{2, \alpha}\left(\Gamma^{*}\right)$. 
The stability property of the stationary solutions is determined by the spectrum of the following associated linearized problem:

$$
\left\{\begin{array}{l}
0=\epsilon^{2} \Delta w+f_{u}^{\epsilon} w-z, \\
0=\Delta z+\epsilon w+\lambda^{\epsilon} w, \\
\frac{\partial w}{\partial n}=0=\frac{\partial z}{\partial n} \quad \text { on } \partial \Omega,
\end{array}\right.
$$

where $f_{u}^{\epsilon}:=\frac{d}{d u} f\left(u^{\epsilon}\right)$. We assume the following for (1.12).

(A6) Each eigenvalue $\lambda^{\epsilon}$ and the associated eigenfunctions $\left(w^{\epsilon}, z^{\epsilon}\right)$ of $(1.12)$ have the following asymptotic forms:

$$
\left\{\begin{array}{l}
\lambda^{\epsilon}=\epsilon \lambda^{*}+o(\epsilon) \\
w^{\epsilon}(x)=\sum_{i=0}^{2} \epsilon^{i} W^{ \pm, i}(x)+\omega(r) \cdot \sum_{i=0}^{2} \epsilon^{i} w^{ \pm, i}(r / \epsilon, y)+o\left(\epsilon^{2}\right), \\
z^{\epsilon}(x)=\sum_{i=0}^{2} \epsilon^{i} Z^{ \pm, i}(x)+\omega(r) \cdot \sum_{j=3}^{5} \epsilon^{j} z^{ \pm, j}(r / \epsilon, y)+o\left(\epsilon^{2}\right),
\end{array}\right.
$$

where $W^{ \pm, i}(x) \in C^{2, \alpha}\left(\bar{\Omega}_{ \pm}\left(\Gamma^{*}\right)\right)$ and $Z^{ \pm, i}(x) \in C^{2, \alpha}\left(\bar{\Omega}_{ \pm}\left(\Gamma^{*}\right)\right)$ are outer expansions, $w^{ \pm, i}(\xi, y)$ and $z^{ \pm, j}(\xi, y)$ are inner expansions bounded for $\pm \xi \in$ $(0, \infty)$, and $y \in \Gamma^{*}, x=(r, y)$ is a local coordinate system in the neighborhood of $\Gamma^{*}$, and $\omega(r)$ is a smooth cutoff function such that

$$
\omega(r)=1, \quad|r| \leq \frac{d_{0}}{2} \omega(r)=0, \quad|r| \geq d_{0} .
$$

For more details, see section 3 .

Here we are interested in only the critical case, i.e., the expansion starts from $\epsilon \lambda^{*}$, which is justified by Lemma 3.1.

Concerning (1.12), we have the following theorem.

THEOREM 1.3 (SLEP equation). Suppose the conditions (A1)-(A6) are valid. Then the principal part of the critical eigenvalues is given by $\epsilon \lambda^{*}$, where $\lambda^{*}$ is the eigenvalue of the following problem:

$$
L \theta^{*}+\frac{1}{m^{2}} \lambda^{*}[h] J^{\prime}\left(v^{*}\right) \mathcal{T}\left(\theta^{*}\right)=0
$$

for $\theta^{*} \in(I-P) C^{2, \alpha}\left(\Gamma^{*}\right)$. [h] denotes the aforementioned jump at $v=v^{*}$.

Theorem 1.3 gives us a general form which characterizes the asymptotic form of critical eigenvalues. When $\Gamma^{*}$ has a special geometry such as spherical or planar shape with $f(u)=u-u^{3}$, then we can rigorously prove that the critical eigenvalues $\lambda^{\epsilon}$ given by the form $\lambda^{\epsilon}=\epsilon \lambda^{*}+o(1)$, and determine the stability properties. More precisely we have the following results (see section 4 for details).

THEOREM 1.4 (stability of radially symmetric patterns). Let $\Omega$ be a ball of radius $R$. Then the following hold:

(i) For any fixed $R \in(0, \infty)$, there exists $\bar{u}_{0}=\bar{u}_{0}(R) \in(-1,1)$ such that the radially symmetric solution is stable for $\bar{u} \in\left(\bar{u}_{0}, 1\right)$. 


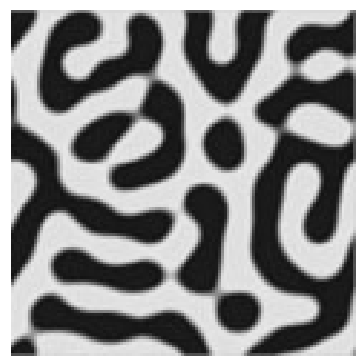

(a)

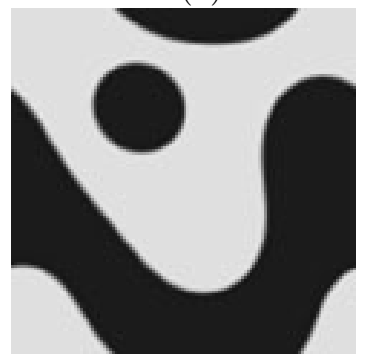

(d)

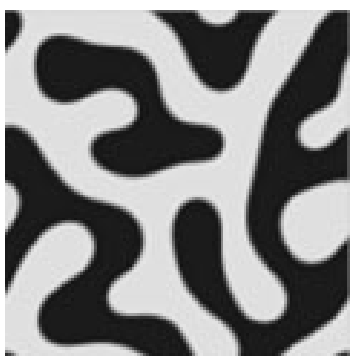

(b)

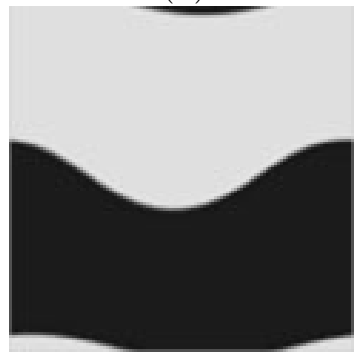

(e)

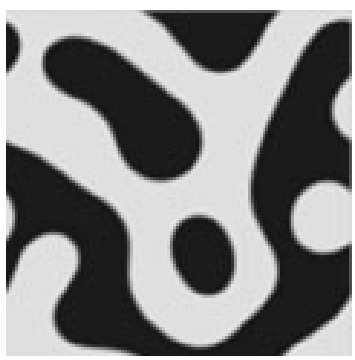

(c)

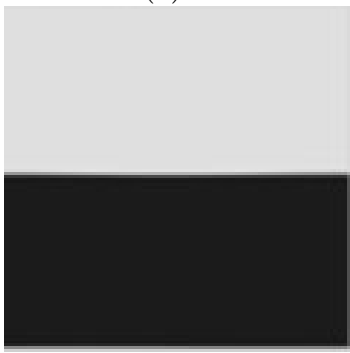

(f)

FIG. 1. Evolution of the interfaces slightly perturbed from the homogeneous state $u=\bar{u}$, where $X=Y=2.0, \epsilon=0.03, \bar{u}=7.10992 \times 10^{-5}$. Since $\bar{u}$ is very close to 0, lamellar shape is preferable: (a) $\tilde{t}=0.05$, (b) $\tilde{t}=0.15$, (c) $\tilde{t}=0.3$, (d) $\tilde{t}=1.5$, (e) $\tilde{t}=6.0$, (f) $\tilde{t}=15.0$.

(ii) For any fixed $\bar{u} \in(-1,1)$, there exists $R_{0}=R_{0}(\bar{u})>0$ such that the radially symmetric solution is unstable for $R>R_{0}$.

Note that here the location of interface is given by $\Gamma^{*}=\left\{x \in \mathbf{R}^{N}|| x \mid=\right.$ $\left.r_{0}\right\}$, where $r_{0}=r_{0}(\bar{u})$ is a monotone decreasing function of $\bar{u}$, and $\Omega_{-}\left(\Gamma^{*}\right)=\{x \in$ $\left.\mathbf{R}^{N}|| x \mid<r_{0}\right\}$. See section 4 for details.

THEOREM 1.5 (stability of planar patterns). Let $\Omega$ be a rectangle $(0, X) \times(0, Y)$ and $\kappa:=X / Y$ be the aspect ratio defined before. Then the following hold:

(i) For any $\bar{u} \in(-1,1)$, there exists $\underline{X}=\underline{X}(\bar{u})>0$ such that the planar solution is stable for any $X<\underline{X}$ and $\kappa>0$.

(ii) For any fixed $\kappa>0$ and $\bar{u} \in(-1,1)$, there exists $\bar{X}=\bar{X}(\kappa, \bar{u})>0$ such that the planar solution is unstable for $X>\bar{X}$.

The results of Theorems 1.4 and 1.5 are useful to understand how the morphology depends on the ratio $\bar{u}$. For instance, Theorem 1.4 (i) implies that the system prefers spherical patterns when either one of the homopolymers dominates the system, which is consistent with the numerics as in Figures 1 and 2. The result of Theorem 1.5 (i) seems against our intuition at first sight; in fact, recalling that the interface is parallel to $y$-axis, it indicates that very long interface in a slender domain with any small aspect ratio can be stabilized, which makes a sharp contrast to the lamellar patterns arising in activator-inhibitor systems (see [22]). This is, however, consistent with the experimental results as well as numerics, because very fine lamellar structure in the original scaling becomes a long strip after rescaling.

The article is organized as follows. The construction of stationary solution of (1.5) and (1.6) is discussed in section 2 by using the matched asymptotic expansion method. In section 3 , the linearized eigenvalue problem (1.12) is reduced to an eigenvalue problem on the interface $\Gamma^{*}$. By using these results, we study the stability properties 


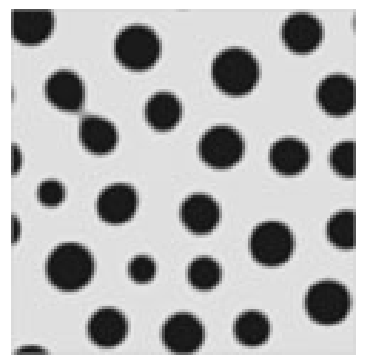

(a)

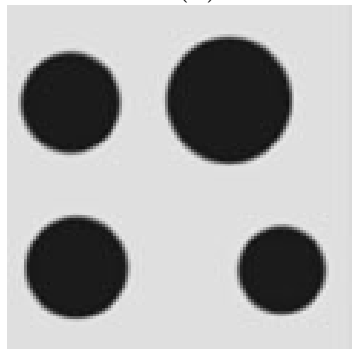

(d)

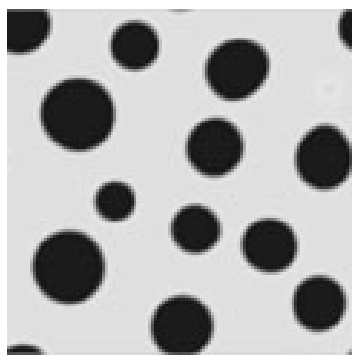

(b)

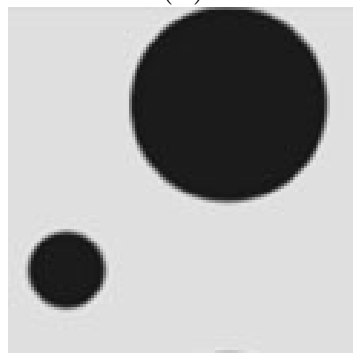

(e)

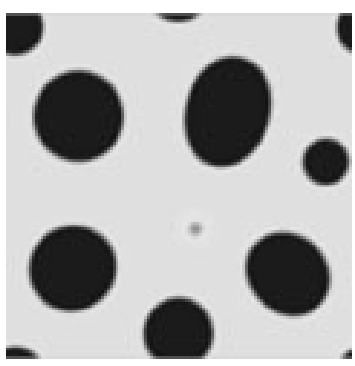

(c)

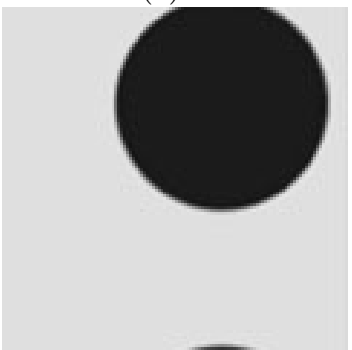

(f)

FIG. 2. Evolution of the interfaces slightly perturbed from the homogeneous state $u=\bar{u}$, where $X=Y=2.0, \epsilon=0.03, \bar{u}=-0.399929$. Since $\bar{u}$ is away from 0, spherical shape is preferable: (a) $\tilde{t}=0.15$, (b) $\tilde{t}=0.5$, (c) $\tilde{t}=1.0$, (d) $\tilde{t}=4.0$, (e) $\tilde{t}=12.0$, (f) $\tilde{t}=15.0$.

of the lamellar and radially symmetric patterns in section 4 . Finally, in section 5, we derive similar results for the activator-inhibitor system (1.4).

\section{Construction of stationary solutions by matched asymptotic expan-} sion. In this section, we prove Theorem 1.2. The strategy is as follows. First, we divide the stationary problem for (1.5) and (1.6) into two problems. That is, for $a_{0}(y), b_{0}(y), b_{1}(y), b_{2}(y) \in C^{2, \alpha}\left(\Gamma^{*}\right)$, consider the following two problems:

$$
\begin{cases}\epsilon^{2} \Delta u^{-}+f\left(u^{-}\right)-v^{-}=0, & \text { in } \Omega_{-}\left(\Gamma^{*}\right), \\ \Delta v^{-}+\epsilon\left(u^{-}-\bar{u}\right)=0, & \\ u^{-}=a_{0}(y), \quad v^{-}=b_{0}(y)+\epsilon b_{1}(y)+\epsilon^{2} b_{2}(y) & \text { on } \Gamma^{*},\end{cases}
$$

$$
\begin{cases}\epsilon^{2} \Delta u^{+}+f\left(u^{+}\right)-v^{+}=0, & \text { in } \Omega_{+}\left(\Gamma^{*}\right), \\ \Delta v^{+}+\epsilon\left(u^{+}-\bar{u}\right)=0, & \text { on } \Gamma^{*}, \\ u^{+}=a_{0}(y), \quad v^{+}=b_{0}(y)+\epsilon b_{1}(y)+\epsilon^{2} b_{2}(y) & \text { on } \partial \Omega . \\ \frac{\partial u^{+}}{\partial n}=0=\frac{\partial v^{+}}{\partial n} & \end{cases}
$$

Here, the interface is regarded as the boundary layer at $\Gamma^{*}$. 
Let $\left(u^{ \pm, \epsilon}(x), v^{ \pm, \epsilon}(x)\right)$ be the solution of $(2.1)_{ \pm}$and define $\left(u^{\epsilon}(x), v^{\epsilon}(x)\right)$ as

$$
\left(u^{\epsilon}(x), v^{\epsilon}(x)\right)= \begin{cases}\left(u^{-, \epsilon}(x), v^{-, \epsilon}(x)\right), & x \in \bar{\Omega}_{-}\left(\Gamma^{*}\right), \\ \left(u^{+, \epsilon}(x), v^{+, \epsilon}(x)\right), & x \in \bar{\Omega}_{+}\left(\Gamma^{*}\right) .\end{cases}
$$

Since they are continuous on $\Gamma^{*}$ and satisfy $(2.1)_{ \pm}$in each domain $\bar{\Omega}_{ \pm}\left(\Gamma^{*}\right)$, they become a stationary solution of (1.5) and (1.6) if and only if their normal derivatives are continuous on $\Gamma^{*}$. So we determine $a_{0}(s), b_{0}(s), b_{1}(s)$, and $b_{2}(s)$ in order that the $C^{1}$-matching condition is satisfied in subsection 3.3. In fact, by taking account of the $C^{1}$-matching condition of order $O(1)$ and $O(\epsilon)$, we can see that $b_{0}(y)=v^{*}$ and $b_{1}(y)=b_{1}^{*}(y)$.

In Theorem 2.1 below, the symbol $C_{\epsilon}^{2, \alpha}(\bar{\Omega})$ stands for the function space $C^{2, \alpha}(\bar{\Omega})$ endowed with the weighted norm defined by

$$
\|u\|_{C_{\epsilon}^{2, \alpha}(\bar{\Omega})}:=\sum_{j=0}^{2} \epsilon^{j}|u|_{j, \bar{\Omega}}+\epsilon^{2+\alpha}|u|_{2+\alpha, \bar{\Omega}},
$$

where

$$
|u|_{j, \bar{\Omega}}=\max _{|\sigma|=j} \sup _{x \in \bar{\Omega}}\left|\partial^{\sigma} u(x)\right|, \quad|u|_{k+\alpha, \bar{\Omega}}=\max _{|\sigma|=k} \sup _{x, y \in \bar{\Omega}} \frac{\left|\partial^{\sigma} u(x)-\partial^{\sigma} u(y)\right|}{|x-y|^{\alpha}}
$$

and $\sigma$ denote the usual multi-indices.

THEOREM 2.1 (Ikeda [9]). Suppose the conditions (A1)-(A4) are valid and set $b_{0}(y)=v^{*}$ and $b_{1}(y)=b_{1}^{*}(y)$. Then for $\epsilon \in\left(0, \epsilon_{0}\right]$ and $a_{0}, b_{2} \in C^{2, \alpha}\left(\Gamma^{*}\right)$ satisfying $a_{0}(y) \in\left(h^{-}\left(v^{*}\right), h^{+}\left(v^{*}\right)\right)$, there exist two families of solutions,

$$
\left(u^{-, \epsilon}, v^{-, \epsilon}\right) \in C_{\epsilon}^{2, \alpha}\left(\bar{\Omega}_{-}\left(\Gamma^{*}\right)\right) \times C^{2, \alpha}\left(\bar{\Omega}_{-}\left(\Gamma^{*}\right)\right)
$$

and

$$
\left(u^{+, \epsilon}, v^{+, \epsilon}\right) \in C_{\epsilon}^{2, \alpha}\left(\bar{\Omega}_{+}\left(\Gamma^{*}\right)\right) \times C^{2, \alpha}\left(\bar{\Omega}_{+}\left(\Gamma^{*}\right)\right)
$$

of $(2.1)_{-}$and $(2.1)_{+}$, respectively, satisfying the following properties:

(1) $\lim _{\epsilon \rightarrow 0} v^{ \pm, \epsilon}(x)=v^{*}$ uniformly on $\bar{\Omega}_{ \pm}\left(\Gamma^{*}\right)$.

(2) For an arbitrary $\delta>0, \lim _{\epsilon \rightarrow 0} u^{ \pm, \epsilon}(x)=h^{ \pm}\left(v^{*}\right)$ uniformly on $\bar{\Omega}_{ \pm}\left(\Gamma^{*}\right) \backslash \Gamma_{\delta}^{*}$.

(3) The solutions $\left(u^{ \pm, \epsilon}, v^{ \pm, \epsilon}\right)$ have an asymptotic characterization as follows: There exists a constant such that the estimates below are valid uniformly in $\epsilon \in\left(0, \epsilon_{0}\right]$ :

$$
\begin{aligned}
& \left\|u^{ \pm, \epsilon}-\mathcal{U}^{ \pm, \epsilon}\right\|_{C_{\epsilon}^{2, \alpha}\left(\bar{\Omega}_{ \pm}\left(\Gamma^{*}\right)\right)} \leq C \epsilon^{3-\alpha}, \\
& \left\|v^{ \pm, \epsilon}-\mathcal{V}^{ \pm, \epsilon}\right\|_{C^{2, \alpha}\left(\bar{\Omega}_{ \pm}\left(\Gamma^{*}\right)\right)} \leq C \epsilon^{3-\alpha},
\end{aligned}
$$

where $\left(\mathcal{U}^{ \pm, \epsilon}, \mathcal{V}^{ \pm, \epsilon}\right)$ are approximate solutions (for more precise information, see (2.7) and the subsequent subsections).

In the next two subsections, we show the procedures of the asymptotic expansion.

2.1. Outer expansion. Substituting the expansion

$$
\begin{aligned}
& U^{ \pm, \epsilon}(x)=U^{ \pm, 0}(x)+\epsilon U^{ \pm, 1}(x)+\epsilon^{2} U^{ \pm, 2}(x), \\
& V^{ \pm, \epsilon}(x)=V^{ \pm, 0}(x)+\epsilon V^{ \pm, 1}(x)+\epsilon^{2} V^{ \pm, 2}(x)
\end{aligned}
$$


into $(2.1)_{ \pm}$and equating like powers of $\epsilon^{k}$, we have the following problem for $\left(U^{ \pm, k}(x)\right.$, $\left.V^{ \pm, k}(x)\right)(k=0,1,2)$ :

$$
\begin{aligned}
& \left\{\begin{array}{l}
f\left(U^{ \pm, 0}\right)-V^{ \pm, 0}=0, \\
\Delta V^{ \pm, 0}=0,
\end{array}\right. \\
& \left\{\begin{array}{l}
f_{u}^{ \pm, 0} U^{ \pm, 1}-V^{ \pm, 1}=0, \\
\Delta V^{ \pm, 1}+U^{ \pm, 0}-\bar{u}=0,
\end{array}\right. \\
& \left\{\begin{array}{l}
f_{u}^{ \pm, 0} U^{ \pm, 2}-V^{ \pm, 2}+\frac{1}{2} f_{u u}^{ \pm, 0}\left(U^{ \pm, 1}\right)^{2}=0, \\
\Delta V^{ \pm, 2}+U^{ \pm, 1}=0,
\end{array}\right.
\end{aligned}
$$

where $f_{u}^{ \pm, 0}=\frac{d}{d u} f\left(U^{ \pm, 0}(x)\right)$ and others are similarly defined. $V^{-, 0}$ and $V^{+, 0}$ are uniquely determined under the boundary conditions

$$
V^{ \pm, 0}=b_{0} \quad \text { on } \Gamma^{*}, \quad \frac{\partial V^{+, 0}}{\partial n}=0 \quad \text { on } \partial \Omega,
$$

respectively. They are represented as $V^{ \pm, 0}=\mathcal{P}^{ \pm} b_{0}$. Then we define $U^{ \pm, 0}$ as

$$
U^{ \pm, 0}=h^{ \pm}\left(V^{ \pm, 0}\right)
$$

where $h^{ \pm}(v)$ is sub-branch of $f(u)-v=0$ defined in section 1 .

The equation for $V^{ \pm, 1}$ with the boundary conditions is recast as

$$
\begin{cases}\Delta V^{ \pm, 1}+h^{ \pm}\left(V^{ \pm, 0}\right)-\bar{u}=0 & \text { in } \Omega_{ \pm}\left(\Gamma^{*}\right), \\ V^{ \pm, 1}=b_{1}(y) & \text { on } \Gamma^{*}, \\ \frac{\partial V^{+, 1}}{\partial n}=0 & \text { on } \partial \Omega,\end{cases}
$$

respectively. It is not difficult to show that $(2.2)_{ \pm}$has a unique solution, $V^{ \pm, 1}$. Then, by using $V^{ \pm, 1}, U^{ \pm, 1}$ are determined as

$$
U^{ \pm, 1}=h_{v}^{ \pm}\left(V^{ \pm, 0}\right) V^{ \pm, 1},
$$

where $h_{v}^{ \pm}(v)=\frac{d}{d v} h^{ \pm}(v)$. As for the equations for $\left(U^{ \pm, 2}, V^{ \pm, 2}\right)$, we obtain the following:

$$
U^{ \pm, 2}(x)=h_{v}^{ \pm}\left(V^{ \pm, 0}\right) V^{ \pm, 2}+\frac{1}{2} h_{v v}^{ \pm}\left(V^{ \pm, 0}\right)\left(V^{ \pm, 1}\right)^{2}
$$

and

$$
\begin{cases}0=\Delta V^{ \pm, 2}+h_{v}^{ \pm}\left(V^{ \pm, 0}\right) V^{ \pm, 1}(x) & \text { in } \Omega_{ \pm}\left(\Gamma^{*}\right), \\ \frac{\partial V^{+, 2}}{\partial n}=0 \quad \text { on } \partial \Omega, \quad V^{ \pm, 2}=b_{2}(y) & \text { on } \Gamma^{*}\end{cases}
$$

The boundary value problems for $V^{ \pm, 2}$ are uniquely solvable for arbitrary $b_{2} \in$ $C^{2, \alpha}\left(\Gamma^{*}\right)$. They are expressed as

$$
V^{ \pm, 2}=\mathcal{P}^{ \pm} b_{2}+W^{ \pm}
$$


where $W^{ \pm} \in C^{2, \alpha}\left(\bar{\Omega}_{ \pm}\left(\Gamma^{*}\right)\right)$ are solutions of

$$
\begin{cases}\Delta W^{ \pm}=-h_{v}^{ \pm}\left(V^{ \pm, 0}(x)\right) V^{ \pm, 1}(x) & \text { in } \Omega_{ \pm}\left(\Gamma^{*}\right), \\ W^{ \pm}=0 \text { on } \Gamma^{*}, \quad \frac{\partial W^{+}}{\partial n}=0 & \text { on } \partial \Omega\end{cases}
$$

respectively. Once $V^{ \pm, 2}$ is known, $U^{ \pm, 2}$ is uniquely determined by the expression above.

In this way, we have obtained the following outer expansion:

$$
\left\{\begin{array}{l}
V^{ \pm, \epsilon}(x)=V^{ \pm, 0}(x)+\epsilon V^{ \pm, 1}(x)+\epsilon^{2}\left(\mathcal{P}^{ \pm} b_{2}+W^{ \pm}\right), \\
U^{ \pm, \epsilon}(x)=h^{ \pm}\left(V^{ \pm, 0}(x)\right)+\epsilon h_{v}^{ \pm}\left(V^{ \pm, 0}(x)\right) V^{ \pm, 1}(x)+\epsilon^{2} U^{ \pm, 2}(x),
\end{array} \quad x \in \bar{\Omega}_{ \pm}\left(\Gamma^{*}\right) .\right.
$$

This expansion is due to the lack of the layer part, in fact, $U^{+, \epsilon}$ and $U^{-, \epsilon}$ are not continuous on $\Gamma^{*}$. So we need a new variable that stretches a neighborhood of the interface.

2.2. Inner expansion. Since the outer expansion $U^{+, \epsilon}$ and $U^{-, \epsilon}$ are not continuous on $\Gamma^{*}$, we need to introduce the stretched variable and make another expansion in the neighborhood of $\Gamma^{*}$.

Let us introduce a local coordinate system in the neighborhood of $\Gamma^{*}$. By the implicit function theorem there exists a $d_{0}>0$ such that the map $p:\left[-d_{0}, d_{0}\right] \times \Gamma^{*} \rightarrow$ $\Gamma_{d_{0}}^{*}$ defined by

$$
p(r, y)=y+r \nu(y)
$$

is a diffeomorphism, where $\Gamma_{d_{0}}^{*}=\left\{x \in \Omega \mid \operatorname{dist}\left(x, \Gamma^{*}\right)<d_{0}\right\}$. Using this diffeomorphism, we identify $x \in \Gamma_{d_{0}}^{*}$ with $(r, y) \in\left[-d_{0}, d_{0}\right] \times \Gamma_{d_{0}}^{*}$ and write $u(r, y)$ for $u(x)$ $\left(x \in \Gamma_{d_{0}}^{*}\right)$. With this representation, the suitable magnification of $\Gamma_{d_{0}}^{*}$ corresponds to the scaling: $r=\epsilon \xi$. In terms of the variables $\xi$ and $y \in \Gamma^{*}$, the equations in (1.5) are recast as

$$
\left\{\begin{array}{l}
0=u_{\xi \xi}+\epsilon(N-1) H(\epsilon \xi, y) u_{\xi}+\epsilon^{2} \Delta(\epsilon \xi) u+f(u)-v \\
0=v_{\xi \xi}+\epsilon(N-1) H(\epsilon \xi, y) v_{\xi}+\epsilon^{2} \Delta(\epsilon \xi) v+\epsilon^{3}(u-\bar{u}) .
\end{array}\right.
$$

Here, $H(r, y)$ stands for the mean curvature of the manifold

$$
\Gamma^{*}(r):=\left\{x \in \Omega \mid x=y+r \nu(y) y \in \Gamma^{*}\right\},
$$

and $\Delta(r)$ for the Laplace-Beltrami operator on $\Gamma^{*}(r)$ (for more details, see Sakamoto $[17])$.

We now determine the functions $u^{ \pm, i}(i=0,1,2), v^{ \pm, j}(j=3,4)$ in the following expressions:

$$
\left\{\begin{array}{l}
u=U^{ \pm, \epsilon}(\epsilon \xi, y)+u^{ \pm, 0}(\xi, y)+\epsilon u^{ \pm, 1}(\xi, y)+\epsilon^{2} u^{ \pm, 2}(\xi, y) \\
v=V^{ \pm, \epsilon}(\epsilon \xi, y)+\epsilon^{3} v^{ \pm, 3}(\xi, y)+\epsilon^{4} v^{ \pm, 4}(\xi, y)
\end{array}\right.
$$

The reason why we go to third order $v^{ \pm, 3}(\xi, y)$ in $\epsilon$ is that the inhomogeneous term of the equation for $v$ appears in $O(\epsilon)$-term. We expand the mean curvature $H(\epsilon \xi, y)$ 
and the Laplace-Beltrami operator $\Delta(\epsilon \xi)$ of manifold $\Gamma^{*}(\epsilon \xi)$ as

$$
\begin{array}{rlrl}
H(\epsilon \xi, y) & =\sum_{i \geq 0} \epsilon^{i} \quad H^{i}(\xi, y), \quad H^{i}(\xi, y):=\left.\frac{1}{i !} \frac{d^{i}}{d \epsilon^{i}} H(\epsilon \xi, y)\right|_{\epsilon=0}, \\
\Delta(\epsilon \xi) & =\sum_{i \geq 0} \epsilon^{i} \quad \Delta^{i}(\xi), & \Delta^{i}(\xi) & :=\left.\frac{1}{i !} \frac{d^{i}}{d \epsilon^{i}} \Delta(\epsilon \xi)\right|_{\epsilon=0} .
\end{array}
$$

Here we take account of $O(1)$-term of the $C^{1}$-matching condition for $v$. It is given by

$$
\frac{\partial V^{-, 0}}{\partial \nu}=\frac{\partial V^{+, 0}}{\partial \nu} \text { on } \Gamma^{*} .
$$

Combining the result of subsection 2.1, we can see that $V^{ \pm, 0}$ is a constant function, that is,

$$
V^{-, 0}(x)=V^{+, 0}(x)=\bar{b}_{0}
$$

and $b_{0}(y)=\bar{b}_{0}$ for some $\bar{b}_{0} \in \mathbf{R}$. In the following, we will use this fact.

Substituting (2.4) and the expansions of $H(\epsilon \xi, y)$ and $\Delta(\epsilon \xi)$ into (2.3), equating like powers of $\epsilon$, we have equations for $u^{ \pm, i}(i=0,1,2), v^{ \pm, j}(j=3,4)$. We present them only for $u^{-, i}, v^{-, j}$ below, and omit the superscript "-" of $u^{-, i}$ and $v^{-, j}$.

The equation for $u^{0}$ is

$$
u_{\xi \xi}^{0}+f\left(h^{-}\left(\bar{b}_{0}\right)+u^{0}\right)-\bar{b}_{0}=0, \quad \xi \in(-\infty, 0) .
$$

In view of the boundary conditions in $(2.1)_{-}$, we impose boundary conditions

$$
u^{0}(0, y)+h^{-}\left(\bar{b}_{0}\right)=a_{0}(y), \lim _{\xi \rightarrow-\infty} u^{0}(\xi, y)=0 .
$$

Let $u^{ \pm, *}(\xi ; v)$ be the unique solution of

$u_{\xi \xi}^{ \pm, *}+f\left(u^{ \pm, *}\right)-v=0, \quad \xi \in \pm(0, \infty), u^{ \pm, *}(0 ; v)=h^{0}(v), \quad \lim _{\xi \rightarrow \pm \infty} u^{ \pm, *}(\xi ; v)=h^{ \pm}(v)$.

By using these functions, $u^{-, 0}(\xi, y)$ is given by

$$
u^{0}(\xi, y)=u^{-, *}\left(s_{0}^{-}(y)+\xi ; \bar{b}_{0}\right)-h^{-}\left(\bar{b}_{0}\right),
$$

where $s_{0}^{-} \in C^{2, \alpha}\left(\Gamma^{*}\right)$ is related to $a_{0} \in C^{2, \alpha}\left(\Gamma^{*}\right)$ via the equation

$$
a_{0}(y)=u^{-, *}\left(s_{0}^{-}(y) ; \bar{b}_{0}\right), \quad y \in \Gamma^{*} .
$$

The equation for $\left(u^{-, 1}, v^{-, 3}\right)$ is

$$
\left\{\begin{array}{l}
0=u_{\xi \xi}^{1}+\tilde{f}_{u} u^{1}+p_{1}(\xi, y), \\
0=v_{\xi \xi}^{3}+u^{0}(\xi, y)
\end{array} \quad \xi \in(-\infty, 0),\right.
$$

where

$$
p_{1}(\xi, y)=(N-1) H(0, y) u_{\xi}^{0}(\xi, y)+\tilde{f}_{u} U^{1}(0, y)-V^{1}(0, y),
$$


$\tilde{f}_{u}=\frac{d}{d u} f\left(u^{-, *}\left(s_{0}(y)+\xi ; \bar{b}_{0}\right)\right)$ and others are similarly defined. We emphasize the fact $p_{1}(\xi, y)$ decays exponentially to zero as $\xi \rightarrow-\infty$ uniformly in $y \in \Gamma^{*}$. If we impose the boundary conditions

$$
u^{1}(0, y)=-U^{-, 1}(0, y)=-h_{v}^{-}\left(v^{*}\right) b_{1}^{*}(y), \lim _{\xi \rightarrow-\infty} u^{1}(\xi, y)=0,
$$

then the first equation of (2.5) has a unique solution given by

$$
u^{1}(\xi, y)=-U^{1}(0, y) \frac{u_{\xi}^{0}(\xi, y)}{u_{\xi}^{0}(0, y)}-u_{\xi}^{0}(\xi, y) \int_{0}^{\xi} \frac{1}{\left[u_{\xi}^{0}(\tau, y)\right]^{2}} \int_{-\infty}^{\tau} p_{1}(s, y) u_{\xi}^{0}(s, y) d s d \tau .
$$

The boundary conditions for $v^{3}$ are

$$
v^{3}(0, y)=0, \lim _{\xi \rightarrow-\infty} v^{3}(\xi, y)=0
$$

and $v^{3}$ is uniquely determined as

$$
v^{3}(\xi, y)=-\int_{-\infty}^{\xi} \int_{-\infty}^{\tau} u^{0}(s, y) d s d \tau
$$

Finally, we treat the equation for $\left(u^{-, 2}, v^{-, 4}\right)$

$$
\left\{\begin{array}{l}
0=u_{\xi \xi}^{2}+\tilde{f}_{u} u^{2}+p_{2}(\xi, y), \\
0=v_{\xi \xi}^{4}+q_{4}(\xi, y),
\end{array} \quad \xi \in(-\infty, 0),\right.
$$

where

$$
\begin{aligned}
p_{2}(\xi, y)= & (N-1) H(0, y) u_{\xi}^{1}+\Delta(0) u^{0}+(N-1) H_{r}(0, y) \xi u_{\xi}^{0} \\
& +\frac{1}{2} \tilde{f}_{u u}\left[U^{1}(0, y)+u^{1}\right]^{2}+\tilde{f}_{u}\left[\xi U_{r}^{1}(0, y)+U^{2}(0, y)+u^{2}\right] \\
& -\left[\xi V_{r}^{1}(0, y)+V^{2}(0, y)\right], \\
q_{4}(\xi, y)= & (N-1) H(0, y) v_{\xi}^{1}+u^{1},
\end{aligned}
$$

and $U_{r}^{1}(0, y)=\frac{\partial}{\partial r} U^{1}(0, y)$ and others are similarly defined. We note the fact that $p_{2}(\xi, y)$ and $q_{4}(\xi, y)$ decay exponentially to zero as $\xi \rightarrow-\infty$ uniformly in $y \in \Gamma^{*}$. If we impose the boundary conditions

$$
\begin{array}{ll}
u^{2}(0, y)=-U^{-, 2}(0, y)=-h_{v}^{-}\left(v^{*}\right) b_{2}(y)-\frac{1}{2} h_{v v}^{-}\left(v^{*}\right)\left(b_{1}(y)\right)^{2}, & \lim _{\xi \rightarrow-\infty} u^{2}(\xi, y)=0, \\
v^{4}(0, y)=0, & \lim _{\xi \rightarrow-\infty} v^{4}(\xi, y)=0,
\end{array}
$$

then the equations in (2.6) have unique solutions given by

$$
u^{2}(\xi, y)=-U^{2}(0, y) \frac{u_{\xi}^{0}(\xi, y)}{u_{\xi}^{0}(0, y)}-u_{\xi}^{0}(\xi, y) \int_{0}^{\xi} \frac{1}{\left[u_{\xi}^{0}(\tau, y)\right]^{2}} \int_{-\infty}^{\tau} p_{2}(s, y) u_{\xi}^{0}(s, y) d s d \tau
$$

and

$$
v^{4}(\xi, y)=-\int_{-\infty}^{\xi} \int_{-\infty}^{\tau} q_{4}(s, y) d s d \tau
$$


The same type of arguments can be applied to $u^{+, i}(i=0,1,2)$ and $v^{+, j}(j=3,4)$, which leads to the following approximation:

$$
\left\{\begin{array}{l}
\mathcal{U}^{ \pm, \epsilon}(x)=U^{ \pm, \epsilon}(x)+\left\{u^{ \pm, 0}(r / \epsilon, y)+\epsilon u^{ \pm, 1}(r / \epsilon, y)+\epsilon^{2} u^{ \pm, 2}(r / \epsilon, y)\right\} \cdot \omega(r) \\
\mathcal{V}^{ \pm, \epsilon}(x)=V^{ \pm, \epsilon}(x)+\left\{\epsilon^{3} v^{ \pm, 3}(r / \epsilon, y)+\epsilon^{4} v^{ \pm, 4}(r / \epsilon, y)\right\} \cdot \omega(r)
\end{array}\right.
$$

where $\omega(r)$ is a smooth cutoff function such that

$$
\omega(r)=1, \quad|r| \leq \frac{d_{0}}{2} \omega(r)=0,|r| \geq d_{0}
$$

2.3. $C^{\mathbf{1}}$-matching of normal derivatives on $\Gamma^{*}$. In this subsection, we make stationary solutions with the internal transition layer on a whole domain $\Omega$ by matching the normal derivatives of $\left(u^{ \pm, \epsilon}(x), v^{ \pm, \epsilon}(x)\right)$ on $\Gamma^{*}$.

The normal derivatives $\mathcal{U}^{ \pm, \epsilon}$ and $\mathcal{V}^{ \pm, \epsilon}$ on $\Gamma^{*}$ are computed as

$$
\begin{aligned}
\left.\frac{\partial}{\partial \nu} \mathcal{U}^{ \pm, \epsilon}\right|_{\Gamma^{*}} & =\epsilon U_{r}^{ \pm, 1}(0, y)+\epsilon^{2} U_{r}^{ \pm, 2}(0, y)+\frac{1}{\epsilon} u_{\xi}^{ \pm, 0}(0, y)+u_{\xi}^{ \pm, 1}(0, y)+\epsilon u_{\xi}^{ \pm, 2}(0, y), \\
\left.\frac{\partial}{\partial \nu} \mathcal{V}^{ \pm, \epsilon}\right|_{\Gamma^{*}} & =\epsilon V_{r}^{*}(0, y)+\epsilon^{2} \frac{\partial}{\partial \nu}\left(\mathcal{P}^{ \pm} b_{2}\right)+\epsilon^{2} W_{r}^{ \pm}(0, y)+\epsilon^{2} v_{\xi}^{ \pm, 3}(0, y)+\epsilon^{3} v_{\xi}^{ \pm, 4}(0, y) .
\end{aligned}
$$

Then $O\left(\frac{1}{\epsilon}\right)$ and $O(1)$-term of $u_{r}^{-, \epsilon}(0, y)-u_{r}^{+, \epsilon}(0, y)$ are given by

$$
u_{\xi}^{-, 0}(0, y)-u_{\xi}^{+, 0}(0, y) \text { and } u_{\xi}^{-, 1}(0, y)-u_{\xi}^{+, 1}(0, y) \text {. }
$$

Multiplying $u_{\xi}^{ \pm, 0}$ by the equation of $u^{ \pm, 0}$ and integrating it from $\pm \infty$ to 0 , we have

$$
\frac{1}{2}\left[u_{\xi}^{ \pm, 0}(0, y)\right]^{2}+\int_{h^{ \pm}\left(\bar{b}_{0}\right)}^{a_{0}(y)}\left[f(u)-\bar{b}_{0}\right] d u=0
$$

Noting that $u^{ \pm, 0}(\xi, y)$ is a monotonically increasing function with respect to $\xi$, we can see that $u_{\xi}^{-, 0}(0, y)-u_{\xi}^{+, 0}(0, y)=0$ is equivalent to

$$
J\left(\bar{b}_{0}\right)=\int_{h^{-}\left(\bar{b}_{0}\right)}^{h^{+}\left(\bar{b}_{0}\right)}\left[f(u)-\bar{b}_{0}\right] d u=0 .
$$

We can conclude from (A3) that

$$
b_{0}(y) \equiv \bar{b}_{0}=v^{*}=0 \text {. }
$$

In the following, we set $\bar{b}_{0}=v^{*}$. Then we can see that $u^{ \pm, 0}(\xi, y)$ is represented as

$$
u^{ \pm, 0}(\xi, y)=u^{*}\left(\xi+s_{0}(y)\right)-h^{ \pm}\left(v^{*}\right)
$$

where $u^{*}(\xi)$ is the function defined in section 1 and $s_{0} \in C^{2, \alpha}\left(\Gamma^{*}\right)$ is a function related to $a_{0} \in C^{2, \alpha}\left(\Gamma^{*}\right)$ via the equation

$$
a_{0}(y)=u^{*}\left(s_{0}(y)\right), \quad y \in \Gamma^{*} .
$$

Also we obtain $s_{0}(y)=s_{0}^{-}(y)=s_{0}^{+}(y)$. 
Since $u_{\xi}^{ \pm, 1}(0, y)$ is computed as

$$
u_{\xi}^{ \pm, 1}(0, y)=-\frac{1}{u_{\xi}^{*}\left(s_{0}\right)} \int_{ \pm \infty}^{0}\left[(N-1) H(0, y) u_{\xi}^{*}\left(\xi+s_{0}(y)\right)-b_{1}(y)\right] u_{\xi}^{*}\left(s+s_{0}\right) d s
$$

we can see that $u_{\xi}^{-, 1}(0, y)-u_{\xi}^{+, 1}(0, y)=0$ is equivalent to

$$
0=\frac{1}{u_{\xi}^{*}\left(s_{0}\right)}\left[(N-1) H(0, y) \int_{-\infty}^{\infty}\left[u_{\xi}^{*}(\xi)\right]^{2} d \xi-b_{1}(y)[h]\right] .
$$

Thus we obtain

$$
b_{1}(y)=b_{1}^{*}(y)=\frac{m^{2}}{[h]}(N-1) H(0, y)
$$

Concerning the normal derivatives of $u^{-, \epsilon}(x)$ and $v^{-, \epsilon}(x)$, we have the following proposition.

Proposition 2.2. Set $b_{0}(y)=v^{*}$ and $b_{1}(y)=b_{1}^{*}(y)$. For the derivatives of $\left(u^{ \pm, \epsilon}(x), v^{ \pm, \epsilon}(x)\right)$ on $\Gamma^{*}$, we have the following relations:

$$
\begin{aligned}
& v_{r}^{-, \epsilon}(0, y)-v_{r}^{+, \epsilon}(0, y)=\epsilon^{2}\left[\Pi_{-} b_{2}+\Pi_{+} b_{2}-s_{0}[h]-\Psi_{0}^{*}\right]+\epsilon^{3-\alpha} R_{1}^{\epsilon}\left(s_{0}, b_{2}\right), \\
& u_{\xi}^{*}\left(s_{0}\right)\left[u_{r}^{-, \epsilon}(0, y)-u_{r}^{+, \epsilon}(0, y)\right] \\
& =\epsilon\left[\left(-m^{2} \Delta(0)-m^{2} H^{*}(y)+J^{\prime}\left(v^{*}\right) V_{r}(0, y)\right) s_{0}-J^{\prime}\left(v^{*}\right) b_{2}-\Phi_{0}^{*}\right] \\
& \quad+\epsilon^{2-\alpha} R_{2}^{\epsilon}\left(s_{0}, b_{2}\right)
\end{aligned}
$$

where

$$
\begin{aligned}
m^{2}= & \int_{-\infty}^{\infty}\left[u_{\xi}^{*}(\xi)\right]^{2} d \xi, \quad H^{*}(y):=-(N-1) \frac{\partial}{\partial r} H(0, y)=\sum_{j=1}^{N-1} \kappa_{j}^{2}, \\
\Psi_{0}^{*}= & W_{r}^{+}(0, y)-W_{r}^{-}(0, y)+\int_{-\infty}^{0}\left[u^{*}(\xi)-h^{-}\left(v^{*}\right)\right] d \xi-\int_{\infty}^{0}\left[u^{*}(\xi)-h^{+}\left(v^{*}\right)\right] d \xi, \\
\Phi_{0}^{*}= & -H^{*}(y) \int_{-\infty}^{\infty} \xi\left[u_{\xi}^{*}(\xi)\right]^{2} d \xi-V_{r}^{1}(0, y) \int_{-\infty}^{\infty} \xi u_{\xi}^{*}(\xi) d \xi \\
& +\int_{-\infty}^{\infty} \xi\left[(N-1) H(0, y) u_{\xi}^{*}(\xi)-b_{1}^{*}(y)\right] u_{\xi}^{*}(\xi) d \xi-\frac{1}{2}\left[b_{1}^{*}(y)\right]^{2}\left[h^{+}\left(v^{*}\right)-h^{-}\left(v^{*}\right)\right],
\end{aligned}
$$

$\kappa_{j}(j=1,2, \ldots, N-1)$ are the principal curvatures of $\Gamma^{*}$. Moreover, $R_{1}^{\epsilon}\left(s_{0}, b_{2}\right)$ and $R_{2}^{\epsilon}\left(s_{0}, b_{2}\right)$ satisfy

$$
\left\|R_{1}^{\epsilon}\left(s_{0}, b_{2}\right)\right\|_{C^{1, \alpha}\left(\Gamma^{*}\right)}=O(1),\left\|R_{2}^{\epsilon}\left(s_{0}, b_{2}\right)\right\|_{C^{\alpha}\left(\Gamma^{*}\right)}=O(1) \quad \text { as } \epsilon \rightarrow 0 .
$$

Proof. See Appendix A.

Lemma 2.3. The operators $R_{1}^{\epsilon}\left(s_{0}, b_{2}\right)$ and $R_{2}^{\epsilon}\left(s_{0}, b_{2}\right)$ are Lipschitz continuous in $\left(s_{0}, b_{2}\right)$. More precisely, there exists a $C>0$, independent of $\epsilon \in\left(0, \epsilon_{0}\right]$, such that

$$
\begin{aligned}
\left\|R_{1}^{\epsilon}\left(s^{1}, b^{1}\right)-R_{1}^{\epsilon}\left(s^{2}, b^{2}\right)\right\|_{C^{1, \alpha}\left(\Gamma^{*}\right)} & \leq C\left[\left\|s^{1}-s^{2}\right\|_{C^{2, \alpha}\left(\Gamma^{*}\right)}+\left\|b^{1}-b^{2}\right\|_{C^{2, \alpha}\left(\Gamma^{*}\right)}\right], \\
\left\|R_{2}^{\epsilon}\left(s^{1}, b^{1}\right)-R_{2}^{\epsilon}\left(s^{2}, b^{2}\right)\right\|_{C^{\alpha}\left(\Gamma^{*}\right)} & \leq C\left[\left\|s^{1}-s^{2}\right\|_{C^{2, \alpha}\left(\Gamma^{*}\right)}+\left\|b^{1}-b^{2}\right\|_{C^{2, \alpha}\left(\Gamma^{*}\right)}\right] .
\end{aligned}
$$


Proof of Lemma 2.3. We can prove this lemma in the same manner as the proof of Theorem 3.1 in [17].

Let us solve the following equations for $s_{0}$ and $b_{2}$ under the conditions $b_{0}(y)=v^{*}$ and $b_{1}(y)=b_{1}^{*}(y)$ :

$$
\left\{\begin{array}{l}
\Phi\left(s_{0}, b_{2}, \epsilon\right):=-\frac{1}{m^{2} \epsilon} u_{\xi}^{*}\left(s_{0}\right)\left[u_{r}^{-, \epsilon}(0, y)-u_{r}^{+, \epsilon}(0, y)\right]=0 \\
\Psi\left(s_{0}, b_{2}, \epsilon\right):=\frac{1}{\epsilon^{2}}\left[v_{r}^{-, \epsilon}(0, y)-v_{r}^{+, \epsilon}(0, y)\right]=0
\end{array}\right.
$$

The next proposition guarantees that the normal derivatives of $u^{ \pm, \epsilon}$ and $v^{ \pm, \epsilon}$ on $\Gamma^{*}$ are matched continuously for an appropriate pair $\left(s_{0}^{\epsilon}, b_{2}^{\epsilon}\right)$.

Proposition 2.4. Suppose the conditions (A1)-(A5) are valid. There exists a pair $\left(s_{0}^{\epsilon}, b_{2}^{\epsilon}\right) \in C^{2, \alpha}\left(\Gamma^{*}\right) \times C^{2, \alpha}\left(\Gamma^{*}\right)$ satisfying $(2.10)$ for small $\epsilon>0$.

Proof. When $\epsilon=0,(2.10)$ can be rewritten as

$$
\left\{\begin{array}{l}
M s+\frac{1}{m^{2}} J^{\prime}\left(v^{*}\right) b+\frac{1}{m^{2}} \Phi_{0}^{*}=0, \\
\Pi b-[h] s-\Psi_{0}^{*}=0
\end{array}\right.
$$

where

$$
M:=\Delta^{\Gamma^{*}}+H^{*}(y)-\frac{1}{m^{2}} J^{\prime}\left(v^{*}\right) V_{r}(0, y) .
$$

Let $P$ be a projection onto the null space $\mathcal{N}(\Pi)$, i.e.,

$$
P a:=\frac{1}{\left|\Gamma^{*}\right|} \int_{\Gamma^{*}} a d S \text { for } a \in C^{2, \alpha}\left(\Gamma^{*}\right) .
$$

Then (2.11) is equivalent to the following system:

$$
\left\{\begin{array}{l}
P M\left(s_{\mathcal{N}}+s_{\dagger}\right)+\frac{1}{m^{2}} J^{\prime}\left(v^{*}\right) b_{\mathcal{N}}+\frac{1}{m^{2}} P \Phi_{0}^{*}=0 \\
(I-P) M\left(s_{\mathcal{N}}+s_{\dagger}\right)+\frac{1}{m^{2}} J^{\prime}\left(v^{*}\right) b_{\dagger}+\frac{1}{m^{2}}(I-P) \Phi_{0}^{*}=0 \\
-[h] s_{\mathcal{N}}-P \Psi_{0}^{*}=0 \\
\Pi b_{\dagger}-[h] s_{\dagger}-(I-P) \Psi_{0}^{*}=0
\end{array}\right.
$$

where $s=s_{\mathcal{N}}+s_{\dagger}, b=b_{\mathcal{N}}+b_{\dagger}, s_{\mathcal{N}}, b_{\mathcal{N}} \in P C^{2, \alpha}\left(\Gamma^{*}\right)$, and $s_{\dagger}, b_{\dagger} \in(I-P) C^{2, \alpha}\left(\Gamma^{*}\right)$. Solving the third and fourth equations in (2.12) with respect to $s_{\mathcal{N}}$ and $b_{\dagger}$, respectively, we have

$$
\left\{\begin{array}{l}
s_{\mathcal{N}}^{*}=-\frac{1}{[h]} P \Psi_{0}^{*}, \\
b_{\dagger}=[h] \mathcal{T} s_{\dagger}+\mathcal{T}(I-P) \Psi_{0}^{*} .
\end{array}\right.
$$

Substituting (2.13) into the first and second equations in (2.12), we obtain

$$
\left\{\begin{array}{l}
P M\left(s_{\mathcal{N}}^{*}+s_{\dagger}\right)+\frac{1}{m^{2}} J^{\prime}\left(v^{*}\right) b_{\mathcal{N}}+\frac{1}{m^{2}} P \Phi_{0}^{*}=0 \\
(I-P) M s_{\dagger}+\frac{1}{m^{2}}[h] J^{\prime}\left(v^{*}\right) \mathcal{T} s_{\dagger}+(I-P) M s_{\mathcal{N}}^{*} \\
\quad+\frac{1}{m^{2}} J^{\prime}\left(v^{*}\right) \mathcal{T}(I-P) \Psi_{0}^{*}+\frac{1}{m^{2}}(I-P) \Phi_{0}^{*}=0
\end{array}\right.
$$


By using the assumption (A5), we can solve the second equation in (2.14) with respect to $s_{\dagger}$.

$$
s_{\dagger}^{*}=-L^{\dagger}\left[(I-P) M s_{\mathcal{N}}^{*}+\frac{1}{m^{2}} J^{\prime}\left(v^{*}\right) \mathcal{T}(I-P) \Psi_{0}^{*}+\frac{1}{m^{2}}(I-P) \Phi_{0}^{*}\right] .
$$

Then $b_{\mathcal{N}}$ and $b_{\dagger}$ are determined as

$$
b_{\mathcal{N}}^{*}=-\frac{1}{J^{\prime}\left(v^{*}\right)}\left[m^{2} P M\left(s_{\mathcal{N}}^{*}+s_{\dagger}^{*}\right)-P \Phi_{0}^{*}\right], \quad b_{\dagger}^{*}=[h] \mathcal{T} s_{\dagger}^{*}+\mathcal{T}(I-P) \Psi_{0}^{*} .
$$

Now we find solutions of (2.10). Substituting

$$
s_{0}=s_{\mathcal{N}}^{*}+s_{\dagger}^{*}+\sigma_{\mathcal{N}}+\sigma_{\dagger} \quad \text { and } \quad b_{2}=b_{\mathcal{N}}^{*}+b_{\dagger}^{*}+\beta_{\mathcal{N}}+\beta_{\dagger}
$$

into (2.10) and operating $P$ and $I-P$ to the equations, we have

$$
\begin{gathered}
P M\left(\sigma_{\mathcal{N}}+\sigma_{\dagger}\right)+\frac{1}{m^{2}} J^{\prime}\left(v^{*}\right) \beta_{\mathcal{N}}-\epsilon^{1-\alpha} P \hat{R}_{2}^{\epsilon}\left(\sigma_{\mathcal{N}}, \sigma_{\dagger}, \beta_{\mathcal{N}}, \beta_{\dagger}\right)=0, \\
(I-P) M\left(\sigma_{\mathcal{N}}+\sigma_{\dagger}\right)+\frac{1}{m^{2}} J^{\prime}\left(v^{*}\right) \beta_{\dagger}-\epsilon^{1-\alpha}(I-P) \hat{R}_{2}^{\epsilon}\left(\sigma_{\mathcal{N}}, \sigma_{\dagger}, \beta_{\mathcal{N}}, \beta_{\dagger}\right)=0, \\
-[h] \sigma_{\mathcal{N}}+\epsilon^{1-\alpha} P \hat{R}_{1}^{\epsilon}\left(\sigma_{\mathcal{N}}, \sigma_{\dagger}, \beta_{\mathcal{N}}, \beta_{\dagger}\right)=0, \\
\Pi \beta_{\dagger}-[h] \sigma_{\dagger}+\epsilon^{1-\alpha}(I-P) \hat{R}_{1}^{\epsilon}\left(\sigma_{\mathcal{N}}, \sigma_{\dagger}, \beta_{\mathcal{N}}, \beta_{\dagger}\right)=0,
\end{gathered}
$$

where

$$
\begin{aligned}
& \hat{R}_{1}^{\epsilon}\left(\sigma_{\mathcal{N}}, \sigma_{\dagger}, \beta_{\mathcal{N}}, \beta_{\dagger}\right)=R_{1}^{\epsilon}\left(s_{\mathcal{N}}^{*}+s_{\dagger}^{*}+\sigma_{\mathcal{N}}+\sigma_{\dagger}, b_{\mathcal{N}}^{*}+b_{\dagger}^{*}+\beta_{\mathcal{N}}+\beta_{\dagger}\right), \\
& \hat{R}_{2}^{\epsilon}\left(\sigma_{\mathcal{N}}, \sigma_{\dagger}, \beta_{\mathcal{N}}, \beta_{\dagger}\right)=\frac{1}{m^{2}} R_{2}^{\epsilon}\left(s_{\mathcal{N}}^{*}+s_{\dagger}^{*}+\sigma_{\mathcal{N}}+\sigma_{\dagger}, b_{\mathcal{N}}^{*}+b_{\dagger}^{*}+\beta_{\mathcal{N}}+\beta_{\dagger}\right) .
\end{aligned}
$$

We can solve (2.17) with respect to $\sigma_{\mathcal{N}}$ when $\epsilon>0$ is small.

$$
\sigma_{\mathcal{N}}=\tilde{\sigma}_{\mathcal{N}}^{\epsilon}\left(\sigma_{\dagger}, \beta_{\mathcal{N}}, \beta_{\dagger}\right)=\frac{\epsilon^{1-\alpha}}{[h]} P \hat{R}_{1}^{\epsilon}\left(\tilde{\sigma}_{\mathcal{N}}^{\epsilon}\left(\sigma_{\dagger}, \beta_{\mathcal{N}}, \beta_{\dagger}\right), \sigma_{\dagger}, \beta_{\mathcal{N}}, \beta_{\dagger}\right) .
$$

Substituting (2.19) into (2.18), we have

$$
\Pi \beta_{\dagger}-[h] \sigma_{\dagger}+\epsilon^{1-\alpha}(I-P) \hat{R}_{1}^{\epsilon}\left(\tilde{\sigma}_{\mathcal{N}}^{\epsilon}\left(\sigma_{\dagger}, \beta_{\mathcal{N}}, \beta_{\dagger}\right), \sigma_{\dagger}, \beta_{\mathcal{N}}, \beta_{\dagger}\right)=0 .
$$

When $\epsilon>0$ is small, (2.20) is solvable in $\beta_{\dagger}$ as

$$
\begin{aligned}
\beta_{\dagger} & =\beta_{\dagger}^{\epsilon}\left(\sigma_{\dagger}, \beta_{\mathcal{N}}\right) \\
& =[h] \mathcal{T} \sigma_{\dagger}-\epsilon^{1-\alpha} \mathcal{T}(I-P) \hat{R}_{1}^{\epsilon}\left(\tilde{\sigma}_{\mathcal{N}}\left(\sigma_{\dagger}, \beta_{\mathcal{N}}, \beta_{\dagger}^{\epsilon}\left(\sigma_{\dagger}, \beta_{\mathcal{N}}\right)\right), \sigma_{\dagger}, \beta_{\mathcal{N}}, \beta_{\dagger}^{\epsilon}\left(\sigma_{\dagger}, \beta_{\mathcal{N}}\right)\right) .
\end{aligned}
$$

By using $(2.21), \sigma_{\mathcal{N}}$ is represented as

$$
\sigma_{\mathcal{N}}=\sigma_{\mathcal{N}}^{\epsilon}\left(\sigma_{\dagger}, \beta_{\mathcal{N}}\right):=\tilde{\sigma}_{\mathcal{N}}^{\epsilon}\left(\sigma_{\dagger}, \beta_{\mathcal{N}}, \beta_{\dagger}^{\epsilon}\left(\sigma_{\dagger}, \beta_{\mathcal{N}}\right)\right) .
$$


Here note that $\sigma_{\mathcal{N}}^{\epsilon}\left(\sigma_{\dagger}, \beta_{\mathcal{N}}\right)$ and $\beta_{\dagger}^{\epsilon}\left(\sigma_{\dagger}, \beta_{\mathcal{N}}\right)$ are Lipschitz continuous in $\left(\sigma_{\dagger}, \beta_{\mathcal{N}}\right)$ since $R_{j}^{\epsilon}\left(s_{0}, b_{2}\right)$ are Lipschitz continuous in $\left(s_{0}, b_{2}\right)$. Moreover, they have the following properties:

$$
\left\{\begin{array}{l}
\left|\sigma_{\mathcal{N}}^{\epsilon}\left(\sigma_{\dagger}^{1}, \beta_{\mathcal{N}}^{1}\right)-\sigma_{\mathcal{N}}^{\epsilon}\left(\sigma_{\dagger}^{2}, \beta_{\mathcal{N}}^{2}\right)\right| \leq \epsilon^{1-\alpha} C\left[\left\|\sigma_{\dagger}^{1}-\sigma_{\dagger}^{2}\right\|_{C^{2, \alpha}\left(\Gamma^{*}\right)}+\epsilon^{1-\alpha}\left|\beta_{\mathcal{N}}^{1}-\beta_{\mathcal{N}}^{2}\right|\right], \\
\left\|\beta_{\dagger}^{\epsilon}\left(\sigma_{\dagger}^{1}, \beta_{\mathcal{N}}^{1}\right)-\beta_{\dagger}^{\epsilon}\left(\sigma_{\dagger}^{2}, \beta_{\mathcal{N}}^{2}\right)\right\|_{C^{2, \alpha}\left(\Gamma^{*}\right)} \leq C\left[\left\|\sigma_{\dagger}^{1}-\sigma_{\dagger}^{2}\right\|_{C^{2, \alpha}\left(\Gamma^{*}\right)}+\epsilon^{1-\alpha}\left|\beta_{\mathcal{N}}^{1}-\beta_{\mathcal{N}}^{2}\right|\right], \\
\left\|B_{\dagger}^{\epsilon}\left(\sigma_{\dagger}^{1}, \beta_{\mathcal{N}}^{1}\right)-B_{\dagger}^{\epsilon}\left(\sigma_{\dagger}^{2}, \beta_{\mathcal{N}}^{2}\right)\right\|_{C^{2, \alpha}\left(\Gamma^{*}\right)} \leq \epsilon^{1-\alpha} C\left[\left\|\sigma_{\dagger}^{1}-\sigma_{\dagger}^{2}\right\|_{C^{2, \alpha}\left(\Gamma^{*}\right)}+\epsilon^{1-\alpha}\left|\beta_{\mathcal{N}}^{1}-\beta_{\mathcal{N} \mid}^{2}\right|\right],
\end{array}\right.
$$

where

$$
\begin{gathered}
B_{\dagger}^{\epsilon}\left(\sigma_{\dagger}, \beta_{\mathcal{N}}\right):=\beta_{\dagger}^{\epsilon}\left(\sigma_{\dagger}, \beta_{\mathcal{N}}\right)-[h] \mathcal{T} \sigma_{\dagger}, \\
\left|\sigma_{\mathcal{N}}^{\epsilon}\right|=O\left(\epsilon^{1-\alpha}\right), \quad\left\|B_{\dagger}^{\epsilon}\right\|_{C^{2, \alpha}\left(\Gamma^{*}\right)}=O\left(\epsilon^{1-\alpha}\right) \text { as } \epsilon \rightarrow 0 .
\end{gathered}
$$

Substituting (2.21) and (2.22) into (2.16), we obtain

$$
\begin{aligned}
(I-P) L \sigma_{\dagger}= & -(I-P) M \sigma_{\mathcal{N}}^{\epsilon}\left(\sigma_{\dagger}, \beta_{\mathcal{N}}\right)-\frac{1}{m^{2}} J^{\prime}\left(v^{*}\right) B_{\dagger}^{\epsilon}\left(\sigma_{\dagger}, \beta_{\mathcal{N}}\right) \\
& +\epsilon^{1-\alpha}(I-P) \hat{R}_{2}^{\epsilon}\left(\sigma_{\mathcal{N}}^{\epsilon}\left(\sigma_{\dagger}, \beta_{\mathcal{N}}\right), \sigma_{\dagger}, \beta_{\mathcal{N}}, \beta_{\dagger}^{\epsilon}\left(\sigma_{\dagger}, \beta_{\mathcal{N}}\right)\right)
\end{aligned}
$$

From the assumption (A5), there exists a constant $C_{0}>0$ such that

$$
\left\|L^{\dagger}\right\|_{C^{\alpha}\left(\Gamma^{*}\right) \longrightarrow C^{2, \alpha}\left(\Gamma^{*}\right)} \leq C_{0}
$$

and (2.24) is recast as

$$
\begin{aligned}
\sigma_{\dagger}= & -L^{\dagger}(I-P) M \sigma_{\mathcal{N}}^{\epsilon}\left(\sigma_{\dagger}, \beta_{\mathcal{N}}\right)-\frac{1}{m^{2}} J^{\prime}\left(v^{*}\right) L^{\dagger} B_{\dagger}^{\epsilon}\left(\sigma_{\dagger}, \beta_{\mathcal{N}}\right) \\
& +\epsilon^{1-\alpha} L^{\dagger}(I-P) \hat{R}_{2}^{\epsilon}\left(\sigma_{\mathcal{N}}^{\epsilon}\left(\sigma_{\dagger}, \beta_{\mathcal{N}}\right), \sigma_{\dagger}, \beta_{\mathcal{N}}, \beta_{\dagger}^{\epsilon}\left(\sigma_{\dagger}, \beta_{\mathcal{N}}\right)\right) .
\end{aligned}
$$

It follows from (2.23) and (2.25) that the right-hand side of (2.26) is a contraction on $\mathbf{B}:=\left\{\sigma_{\dagger} \in(I-P) C^{2, \alpha}\left(\Gamma^{*}\right) \mid\left\|\sigma_{\dagger}\right\|_{C^{2, \alpha}\left(\Gamma^{*}\right)} \leq 1\right\}$ with Lipschitz constant $O\left(\epsilon^{1-\alpha}\right)$. Therefore, (2.26) has a unique solution $\sigma_{\dagger}=\sigma_{\dagger}^{\epsilon}\left(\beta_{\mathcal{N}}\right) \in \mathbf{B}$ with the property,

$$
\left\|\sigma_{\dagger}^{\epsilon}\right\|_{C^{2, \alpha}\left(\Gamma^{*}\right)}=O\left(\epsilon^{1-\alpha}\right) \text { as } \epsilon \rightarrow 0 .
$$

Finally, substituting $(2.21),(2.22)$, and $\sigma_{\dagger}=\sigma_{\dagger}^{\epsilon}\left(\beta_{\mathcal{N}}\right)$ into $(2.15)$, we obtain an equation of $\beta_{\mathcal{N}}$, which is solvable in $\beta_{\mathcal{N}}$.

Proof of Theorem 1.2. By virtue of Theorem 2.1 and Proposition 2.4, we obtain the statements of Theorem 1.2.

3. Linearized eigenvalue problem. In this section, we study the linearized eigenvalue problem around a stationary solution $\left(u^{\epsilon}(x), v^{\epsilon}(x)\right)$,

$$
\begin{cases}0=\epsilon^{2} \Delta w+f_{u}^{\epsilon} w-z, & \text { in } \Omega \\ 0=\Delta z+\epsilon w+\lambda^{\epsilon} w, & \text { on } \partial \Omega \\ \frac{\partial w}{\partial n}=0=\frac{\partial z}{\partial n} & \end{cases}
$$


Our goal in this section is to prove Theorem 1.3. Roughly speaking, the SLEP equation (1.13) in Theorem 1.3 becomes a sufficient condition for $C^{1}$-matching conditions of the concerning eigenfunctions.

We first divide (3.1) into the following two parts:

$$
\begin{aligned}
& \begin{cases}\epsilon^{2} \Delta w^{-, \epsilon}+f_{u}^{\epsilon} w^{-, \epsilon}-z^{-, \epsilon}=0, & \text { in } \Omega_{-}\left(\Gamma^{*}\right), \\
\Delta z^{-, \epsilon}+\epsilon w^{-, \epsilon}+\lambda^{\epsilon} w^{-, \epsilon}=0, & y \in \Gamma^{*}, \\
w^{-, \epsilon}(y)=\Theta^{\epsilon}(y), \quad z^{-, \epsilon}(y)=q^{\epsilon}(y), & \text { in } \Omega_{+}\left(\Gamma^{*}\right),\end{cases} \\
& \begin{cases}\epsilon^{2} \Delta w^{+, \epsilon}+f_{u}^{\epsilon} w^{+, \epsilon}-z^{+, \epsilon}=0, & \text { on } \partial \Omega, \\
\Delta z^{+, \epsilon}+\epsilon w^{+, \epsilon}+\lambda^{\epsilon} w^{+, \epsilon}=0, & \partial w^{+, \epsilon}=0=\frac{\partial z^{+, \epsilon}}{\partial n} \\
\frac{\partial n}{w^{+, \epsilon}}(y)=\Theta^{\epsilon}(y), \quad z^{+, \epsilon}(y)=\Gamma^{\epsilon}(y), & y \in \Gamma^{*},\end{cases}
\end{aligned}
$$

where

$$
q^{\epsilon}(y)=q_{0}(y)+\epsilon q_{1}(y)+\epsilon^{2} q_{2}(y), \quad \lambda^{\epsilon}=\epsilon \lambda_{1} .
$$

$\Theta^{\epsilon}, q_{0}, q_{1}, q_{2} \in C^{2, \alpha}\left(\Gamma^{*}\right)$ are unknown boundary data and $\lambda_{1} \in \mathbf{C}$ are regarded as parameters. Here we choose $\epsilon \lambda_{1}$ as the principal term in order that we can successively expand $(3.2)_{ \pm}$and determine the parameters one after another. In fact, we have the following lemma:

Lemma 3.1. Suppose that $\lambda^{\epsilon}=\lambda_{0}+\epsilon \lambda_{1}$. Then, if we can expand $(3.2)_{ \pm}$and determine the parameters $\Theta^{\epsilon}(y), q^{\epsilon}(y)$ and $\lambda^{\epsilon}$ one after another, we have either Re $\lambda_{0}<0$ or $\lambda_{0}=0$.

Proof. This result is proved by using the formal matched asymptotic expansions starting from $O(1)$. See Appendix B.

Let us first construct the solutions $\left(w^{ \pm, \epsilon}, z^{ \pm, \epsilon}\right)$ of $(3.2)_{ \pm}$, namely, the following.

Theorem 3.2 (see [9]). Suppose the conditions (A1)-(A5) are valid. Then, for $\epsilon \in\left(0, \epsilon_{0}\right], \lambda_{1} \in \mathbf{C}, \Theta^{\epsilon}=\Theta \in C^{2, \alpha}\left(\Gamma^{*}\right), q_{0}, q_{1}, q_{2} \in C^{2, \alpha}\left(\Gamma^{*}\right)$, there exist two families of solutions

$$
\left(w^{-, \epsilon}, z^{-, \epsilon}\right) \in C_{\epsilon}^{2, \alpha}\left(\bar{\Omega}_{-}\left(\Gamma^{*}\right)\right) \times C^{2, \alpha}\left(\bar{\Omega}_{-}\left(\Gamma^{*}\right)\right)
$$

and

$$
\left(w^{+, \epsilon}, z^{+, \epsilon}\right) \in C_{\epsilon}^{2, \alpha}\left(\bar{\Omega}_{+}\left(\Gamma^{*}\right)\right) \times C^{2, \alpha}\left(\bar{\Omega}_{+}\left(\Gamma^{*}\right)\right)
$$

of $(3.2)_{-}$and $(3.2)_{+}$, respectively, which have the following asymptotic characterization: There exists a constant $C>0$ such that the estimates below are valid uniformly in $\epsilon \in\left(0, \epsilon_{0}\right]$ :

$$
\begin{gathered}
\left\|w^{ \pm, \epsilon}-\mathcal{W}_{2}^{ \pm, \epsilon}\right\|_{C_{\epsilon}^{2, \alpha}\left(\bar{\Omega}_{ \pm}\left(\Gamma^{*}\right)\right)} \leq C \epsilon^{3-\alpha}, \\
\left\|z^{ \pm, \epsilon}-\mathcal{Z}_{2}^{ \pm, \epsilon}\right\|_{C^{2, \alpha}\left(\bar{\Omega}_{ \pm}\left(\Gamma^{*}\right)\right)} \leq C \epsilon^{3-\alpha},
\end{gathered}
$$

where $\left(\mathcal{W}_{2}^{ \pm, \epsilon}, \mathcal{Z}_{2}^{ \pm, \epsilon}\right)$ are approximate solutions (see (3.18) for the details). Here $C_{\epsilon}^{2, \alpha}$ is the same Banach space defined in section 2. 
In the next two subsections, we only construct the approximate solutions of (3.2) and omit the superscript (or subscript) + (or - ).

3.1. Outer expansion. Let us substitute

$$
w(x)=W^{0}(x)+\epsilon W^{1}(x)+\epsilon^{2} W^{2}(x), \quad z(x)=Z^{0}(x)+\epsilon Z^{1}(x)+\epsilon^{2} Z^{2}(x)
$$

into (3.2) - and equate like powers of $\epsilon$. Then we have the following problem for $W^{-, i}(x)$ and $Z^{-, j}(x)(i=0,1,2)$ :

$$
\begin{gathered}
\left\{\begin{array}{l}
f_{u}^{0} W^{0}-Z^{0}=0, \\
\Delta Z^{0}=0,
\end{array}\right. \\
\left\{\begin{array}{l}
f_{u}^{0} W^{1}+f_{u}^{1} W^{0}-Z^{1}=0, \\
\Delta Z^{1}+\left(1+\lambda_{1}\right) W^{0}=0,
\end{array}\right. \\
\left\{\begin{array}{l}
f_{u}^{0} W^{2}+f_{u}^{1} W^{j}+f_{u}^{2} W^{0}+\Delta W^{0}-Z^{2}=0, \\
\Delta Z^{2}=-\left(1+\lambda_{1}\right) W^{1},
\end{array}\right.
\end{gathered}
$$

where

$$
f_{u}^{i}:=\left.\frac{1}{i !} \frac{d^{i}}{d \epsilon^{i}} f_{u}\left(\sum_{i=0}^{2} \epsilon^{i} U^{i}(x)\right)\right|_{\epsilon=0} .
$$

$Z^{0}$ is uniquely determined under the boundary condition

$$
Z^{0}=q_{0} \quad \text { on } \Gamma^{*} .
$$

That is represented as $Z^{0}=\mathcal{P}^{-} q_{0}$. By using $Z^{0}, W^{0}$ is determined as

$$
W^{0}=\frac{1}{f_{u}^{0}} Z^{0}=h_{v}^{-}\left(v^{*}\right) Z^{0} .
$$

Here we used the fact that $f\left(h^{-}(v)\right)-v=0$ and $f_{u}^{0}=f_{u}\left(h^{-}\left(v^{*}\right)\right) \neq 0$ (see (A1)). Then, (3.3) can be rewritten as

$$
\left\{\begin{array}{l}
W^{1}=\frac{1}{f_{u}^{0}}\left[-f_{u}^{1} W^{0}+Z^{1}\right], \\
\Delta Z^{1}=-\left(1+\lambda_{1}\right) h_{v}^{-}\left(v^{*}\right) Z^{0},
\end{array}\right.
$$

Once $W^{0}$ and $Z^{0}$ are known, the second equation of (3.5) is Poisson equation associated with $Z^{1}$. Therefore, $Z^{1}$ is uniquely determined under the boundary conditions

$$
Z^{1}=q_{1} \quad \text { on } \Gamma^{*} .
$$

That is represented as

$$
Z^{1}=\mathcal{P}^{-} q_{1}+\hat{Z}^{1}
$$


where $\hat{Z}^{1}$ is the unique solution of

$$
\begin{cases}\Delta \hat{Z}^{1}=-\left(1+\lambda_{1}\right) h_{v}^{-}\left(v^{*}\right) Z^{0} & \text { in } \Omega_{-}\left(\Gamma^{*}\right) \\ \hat{Z}^{1}=0 & \text { on } \Gamma^{*}\end{cases}
$$

Once $Z^{1}$ is known, $W^{1}$ is uniquely determined by the first equation of (3.5).

Noting that the boundary conditions for $Z^{2}$ is given by $Z^{2}=q_{2}$ on $\Gamma^{*}$, we can solve $Z^{2}$ as

$$
Z^{2}=\mathcal{P}^{-} q_{2}+\hat{Z}^{2}
$$

where $\hat{Z}^{2}$ is the unique solutions of

$$
\begin{cases}\Delta \hat{Z}^{2}=-\left(1+\lambda_{1}\right) \frac{Z^{1}-f_{u}^{1} W^{0}}{f_{u}^{0}} & \text { in } \Omega_{-}\left(\Gamma^{*}\right), \\ \hat{Z}^{2}=0 & \text { on } \Gamma^{*}\end{cases}
$$

Once $Z^{2}$ is known, $W^{2}$ is uniquely determined by the first equation of (3.4).

In this way, we have obtained the following outer expansion of order $O\left(\epsilon^{2}\right)$ :

$$
\begin{cases}Z_{2}^{-, \epsilon}(x)=\mathcal{P}^{-} q_{0}+\sum_{i=1}^{2} \epsilon^{i}\left(\mathcal{P}^{-} q_{i}+\hat{Z}^{i}\right) . & \\ W_{2}^{-, \epsilon}(x)=\sum_{j=0}^{2} \epsilon^{j} W^{j}(x), & x \in \bar{\Omega}_{-}\left(\Gamma^{*}\right) .\end{cases}
$$

In the same way as above, we can obtain an outer expansion also for (3.2) $)_{+}$. Since $W_{2}^{+, \epsilon}$ and $W_{2}^{-, \epsilon}$ are not continuous on $\Gamma^{*}$, we introduce a new variable $\xi=r / \epsilon$ and construct the inner part.

3.2. Inner expansion. In terms of the variables $\xi$ and $y \in \Gamma^{*}$, the equations in (3.1) are recast as

$$
\left\{\begin{array}{l}
w_{\xi \xi}+\epsilon(N-1) H(\epsilon \xi, y) w_{\xi}+\epsilon^{2} \Delta(\epsilon \xi) w+f_{u}^{\epsilon} w-z=0, \\
z_{\xi \xi}+\epsilon(N-1) H(\epsilon \xi, y) z_{\xi}+\epsilon^{2} \Delta(\epsilon \xi) z+\epsilon^{3} w+\epsilon^{2} \lambda^{\epsilon} w=0 .
\end{array}\right.
$$

We now determine the functions $w^{-, i}(i=0,1,2), z^{-, j}(j=0,1, \ldots, 5)$ in the following expressions:

$$
\begin{aligned}
& w=\sum_{i=0}^{2} \epsilon^{i} W^{i}(\epsilon \xi, y)+\sum_{i=0}^{2} \epsilon^{i} w^{i}(\xi, y)=\sum_{i=0}^{2} \epsilon^{i} \tilde{W}^{i}(\xi, y)+\sum_{i=0}^{2} \epsilon^{i} w^{i}(\xi, y) \\
& z=\sum_{i=0}^{2} \epsilon^{i} Z^{i}(\epsilon \xi, y)+\sum_{i=0}^{5} \epsilon^{i} z^{i}(\xi, y)=\sum_{i=0}^{2} \epsilon^{i} \tilde{Z}^{i}(\xi, y)+\sum_{i=0}^{5} \epsilon^{i} z^{i}(\xi, y)
\end{aligned}
$$

where

$$
\tilde{W}^{i}:=\left.\frac{1}{i !} \frac{d^{i}}{d \epsilon^{i}}\left(\sum_{k=0}^{2} \epsilon^{k} W^{k}(\epsilon \xi, y)\right)\right|_{\epsilon=0}, \quad \tilde{Z}^{i}:=\left.\frac{1}{i !} \frac{d^{i}}{d \epsilon^{i}}\left(\sum_{k=0}^{2} \epsilon^{k} Z^{k}(\epsilon \xi, y)\right)\right|_{\epsilon=0} .
$$


Also we expand the mean curvature $H(\epsilon \xi, y)$ and the Laplace-Beltrami operator $\Delta(\epsilon \xi)$ of manifold $\Gamma^{*}(\epsilon \xi)$ as in subsection 2.2. Substituting (3.7) into (3.6) and equating like powers of $\epsilon$, we have equations for $w^{-, i}(i=0,1,2)$ and $z^{-, j}(j=0,1, \ldots, 5)$ as follows:

$$
\begin{gathered}
\begin{cases}w_{\xi \xi}^{0}+\tilde{f}_{u}^{0} w^{0}+\tilde{f}_{u}^{0} \tilde{W}^{0}-\left(\tilde{Z}^{0}+z^{0}\right)=0, & \xi \in(-\infty, 0), \\
w^{0}(0, y)=\Theta(y)-W^{0}(0, y), & \lim _{\xi \rightarrow-\infty} w^{0}(\xi, y)=0,\end{cases} \\
\left\{\begin{array}{r}
w_{\xi \xi}^{1}+\tilde{f}_{u}^{0} w^{1}+(N-1) H(0, y) w_{\xi}^{0}+\tilde{f}_{u}^{0} \tilde{W}^{1}+\tilde{f}_{u}^{1}\left(\tilde{W}^{0}+w^{0}\right)-\left(\tilde{Z}^{1}+z^{1}\right)=0, \\
\xi \in(-\infty, 0), \\
w^{1}(0, y)=-W^{1}(0, y),
\end{array} \lim _{\xi \rightarrow-\infty} w^{1}(\xi, y)=0,\right.
\end{gathered}
$$

$$
\begin{cases}w_{\xi \xi}^{2}+\tilde{f}_{u}^{0} w^{2}+(N-1) \sum_{i+j=1} H^{i} w_{\xi}^{j}+\Delta^{0} w^{0}+(\text { value of } & \left.\Delta W^{0} \text { on } \Gamma^{*}\right) \\ +\tilde{f}_{u}^{0} \tilde{W}^{2}+\sum_{i+j=2, i \geq 1} \tilde{f}_{u}^{i}\left(\tilde{W}^{j}+w^{j}\right)-\left(\tilde{Z}^{2}+z^{2}\right)=0, & \xi \in(-\infty, 0) \\ w^{2}(0, y)=-W^{2}(0, y), & \lim _{\xi \rightarrow-\infty} w^{2}(\xi, y)=0\end{cases}
$$

where

$$
\begin{aligned}
& \tilde{f}_{u}^{i}:=\left.\frac{1}{i !} \frac{d^{i}}{d \epsilon^{i}} f_{u}\left(U^{ \pm, \epsilon}(\epsilon \xi, y)+\sum_{j=0}^{2} \epsilon^{j} u^{ \pm, j}(\xi, y)\right)\right|_{\epsilon=0} \quad(i=0,1,2) \\
& \begin{cases}z_{\xi \xi}^{0}=0, & \xi \in(-\infty, 0) \\
z^{0}(0, y)=0, & \lim _{\xi \rightarrow-\infty} z^{0}(\xi, y)=0\end{cases}
\end{aligned}
$$$$
\begin{cases}z_{\xi \xi}^{1}+(N-1) H(0, y) z_{\xi}^{0}=0, & \xi \in(-\infty, 0), \\ z^{1}(0, y)=0, & \lim _{\xi \rightarrow-\infty} z^{1}(\xi, y)=0\end{cases}
$$

$$
\begin{cases}z_{\xi \xi}^{2}+(N-1)\left(H^{0} z_{\xi}^{1}+H^{1} z_{\xi}^{0}\right)+\Delta^{0} z^{0}=0, & \xi \in(-\infty, 0), \\ z^{2}(0, y)=0, & \lim _{\xi \rightarrow-\infty} z^{2}(\xi, y)=0,\end{cases}
$$

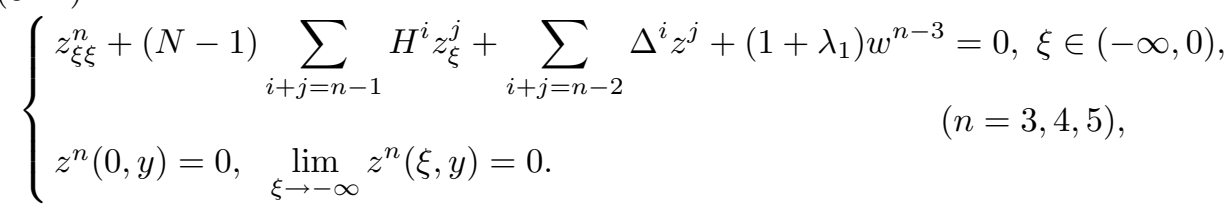


The equations (3.9), (3.10), and (3.11) imply that $z^{j}(\xi, y) \equiv 0(j=0,1,2)$.

The equation for $w^{0}$ is recast as

$$
w_{\xi \xi}^{0}+\tilde{f}_{u}^{0} w^{0}+P_{0}(\xi, y)=0, \quad \xi \in(-\infty, 0)
$$

where

$$
P_{0}(\xi, y)=\tilde{f}_{u}^{0} W^{0}(0, y)-q_{0}(y), \quad \tilde{f}_{u}^{0}=f_{u}\left(u^{*}\left(\xi+s_{0}(y)\right)\right)
$$

In view of the boundary conditions in $(3.2)_{-}$, we impose boundary conditions

$$
w^{0}(0, y)=\Theta(y)-W^{0}(0, y), \quad \lim _{\xi \rightarrow-\infty} w^{0}(\xi, y)=0 .
$$

By using the fact that $u_{\xi}^{*}\left(\xi+s_{0}(y)\right)$ is a fundamental solution of $(3.13), w^{0}$ is uniquely determined as

$$
\begin{aligned}
w^{0}(\xi, y)= & {\left[\Theta(y)-W^{0}(0, y)\right] \frac{u_{\xi}^{*}\left(\xi+s_{0}\right)}{u_{\xi}^{*}\left(s_{0}\right)} } \\
& -u_{\xi}^{*}\left(\xi+s_{0}\right) \int_{0}^{\xi} \frac{1}{\left[u_{\xi}^{*}\left(\tau+s_{0}\right)\right]^{2}} \int_{-\infty}^{\tau} P_{0}(s, y) u_{\xi}^{*}\left(s+s_{0}\right) d s d \tau
\end{aligned}
$$

with $s_{0}=s_{0}(y)$. Noting that $w^{0}$ decays exponentially to zero as $\xi \rightarrow-\infty$, we can solve $z^{3}$ as

$$
z^{3}(\xi, y)=-\left(1+\lambda_{1}\right) \int_{-\infty}^{\xi} \int_{-\infty}^{\tau} w^{0}(s, y) d s d \tau
$$

The equations for $\left(w^{1}, z^{4}\right)$ are

$$
\left\{\begin{array}{l}
0=w_{\xi \xi}^{1}+\tilde{f}_{u} w^{1}+P_{1}(\xi, y), \\
0=z_{\xi \xi}^{4}+Q_{1}(\xi, y),
\end{array} \quad \xi \in(-\infty, 0),\right.
$$

where

$$
\begin{aligned}
& P_{1}(\xi, y)=(N-1) H(0, y) w_{\xi}^{0}+\tilde{f}_{u}^{0} \tilde{W}^{1}+\tilde{f}_{u}^{1}\left(\tilde{W}^{0}+w^{0}\right)-\tilde{Z}^{1}, \\
& Q_{1}(\xi, y)=(N-1) H(0, y) z_{\xi}^{3}+\left(1+\lambda_{1}\right) w^{1} .
\end{aligned}
$$

We emphasize the fact $P_{1}(\xi, y)$ decays exponentially to zero as $\xi \rightarrow-\infty$ uniformly in $y \in \Gamma^{*}$. Therefore, the first equation in (3.16) has a unique solution given by

$$
\begin{aligned}
w^{1}(\xi, y)= & -W^{1}(0, y) \frac{u_{\xi}^{*}\left(\xi+s_{0}\right)}{u_{\xi}^{*}\left(s_{0}\right)} \\
& -u_{\xi}^{*}\left(\xi+s_{0}\right) \int_{0}^{\xi} \frac{1}{\left[u_{\xi}^{*}\left(\tau+s_{0}\right)\right]^{2}} \int_{-\infty}^{\tau} P_{1}(s, y) u_{\xi}^{*}\left(s+s_{0}\right) d s d \tau
\end{aligned}
$$

with $s_{0}=s_{0}(y)$. Once $z^{3}$ and $w^{1}$ are determined, $z^{4}$ is uniquely determined as

$$
z^{4}(\xi, y)=-\int_{-\infty}^{\xi} \int_{-\infty}^{\tau} Q_{1}(s, y) d s d \tau
$$


Here we used the fact that $Q_{1}(\xi, y)$ decays exponentially to zero as $\xi \rightarrow-\infty$ uniformly in $y \in \Gamma^{*}$.

By using the above results, $w^{2}$ and $z^{5}$ are solved as

$$
\begin{aligned}
w^{2}(\xi, y)= & -W^{2}(0, y) \frac{u_{\xi}^{*}\left(\xi+s_{0}\right)}{u_{\xi}^{*}\left(s_{0}\right)} \\
& -u_{\xi}^{*}\left(\xi+s_{0}\right) \int_{0}^{\xi} \frac{1}{\left[u_{\xi}^{*}\left(\tau+s_{0}\right)\right]^{2}} \int_{-\infty}^{\tau} P_{2}(s, y) u_{\xi}^{*}\left(s+s_{0}\right) d s d \tau, \\
z^{5}(\xi, y)= & -\int_{-\infty}^{\xi} \int_{-\infty}^{\tau} Q_{2}(s, y) d s d \tau .
\end{aligned}
$$

with $s_{0}=s_{0}(y)$. Here we used the fact $P_{2}(\xi, y)$ and $Q_{5}(\xi, y)$ decays exponentially to zero as $\xi \rightarrow-\infty$ uniformly in $y \in \Gamma^{*}$.

The same type of arguments as above apply to $w^{+, i}(i=0,1,2)$ and $z^{+, j}$ $(j=0,1, \ldots, 5)$. Now we have obtained the approximation of order $O\left(\epsilon^{2}\right)$,

$$
\left\{\begin{array}{l}
\mathcal{W}_{2}^{ \pm, \epsilon}(x)=W_{2}^{ \pm, \epsilon}(x)+\omega(r) \cdot \sum_{i=0}^{2} \epsilon^{i} w^{ \pm, i}(r / \epsilon, y), \\
\mathcal{Z}_{2}^{ \pm, \epsilon}(x)=Z_{2}^{ \pm, \epsilon}(x)+\omega(r) \cdot \sum_{j=3}^{5} \epsilon^{j} z^{ \pm, j}(r / \epsilon, y),
\end{array}\right.
$$

where $\omega(r)$ is a smooth cutoff function such that

$$
\omega(r)=1,|r| \leq \frac{d_{0}}{2} \omega(r)=0,|r| \geq d_{0} .
$$

3.3. Matching of normal derivatives on $\Gamma^{*}$. Now we are ready to make the eigenfunctions on a whole domain by matching the normal derivatives of $\left(w^{ \pm, \epsilon}, z^{ \pm, \epsilon}\right)$. That is, $\theta(y):=\Theta(y) / u_{\xi}^{*}\left(s_{0}(y)\right), q_{i}(y)(i=0,1,2) \in C^{2, \alpha}\left(\Gamma^{*}\right)$, and $\lambda_{1} \in \mathbf{C}$ must satisfy the following $C^{1}$-matching conditions:

$$
\Phi\left(\theta, q_{0}, q_{1}, q_{2}, \lambda_{1}, \epsilon\right)=0, \quad \Psi\left(\theta, q_{0}, q_{1}, q_{2}, \lambda_{1}, \epsilon\right)=0 \quad \text { on } \Gamma^{*},
$$

where

$$
\begin{aligned}
\Phi\left(\theta, q_{0}, q_{1}, q_{2}, \lambda_{1}, \epsilon\right)(y)= & \epsilon u_{\xi}^{*}\left(s_{0}(y)\right)\left[w_{r}^{-, \epsilon}(0, y)-w_{r}^{+, \epsilon}(0, y)\right] \\
= & u_{\xi}^{*}\left(s_{0}(y)\right)\left[w_{\xi}^{-, 0}(0, y)-w_{\xi}^{+, 0}(0, y)\right] \\
& +u_{\xi}^{*}\left(s_{0}(y)\right) \sum_{i=0}^{1} \epsilon^{i+1}\left[W_{r}^{-, i}(0, y)+w_{\xi}^{-, i+1}(0, y)\right. \\
& \left.-W_{r}^{+, i}(0, y)-w_{\xi}^{+, i+1}(0, y)\right]+\epsilon^{3-\alpha} R_{2}^{\epsilon}\left(\theta, q_{0}, q_{1}, q_{2}, \lambda_{1}\right), \\
\Psi\left(\theta, q_{0}, q_{1}, q_{2}, \lambda_{1}, \epsilon\right)(y)= & z_{r}^{-, \epsilon}(0, y)-z_{r}^{+, \epsilon}(0, y) \\
= & Z_{r}^{-, 0}(0, y)-Z_{r}^{+, 0}(0, y)+\epsilon\left[Z_{r}^{-, 1}(0, y)-Z_{r}^{+, 1}(0, y)\right] \\
& +\epsilon^{2}\left[Z_{r}^{-, 2}(0, y)+z_{\xi}^{-, 3}(0, y)-Z_{r}^{+, 2}(0, y)-z_{\xi}^{+, 3}(0, y)\right] \\
& +\epsilon^{3-\alpha} R_{1}^{\epsilon}\left(\theta, q_{0}, q_{1}, q_{2}, \lambda_{1}\right) .
\end{aligned}
$$


The crucial part of (3.19) will turn out to be $O\left(\epsilon^{2}\right)$, which leads to the conclusion (1.13). We first compute $O(1)$ and $O(\epsilon)$ terms of (3.19).

Lemma 3.3. When $\epsilon=0,(3.19)$ is equivalent to

$$
\begin{gathered}
u_{\xi}^{*}\left(s_{0}(y)\right)\left[w_{\xi}^{-, 0}(0, y)-w_{\xi}^{+, 0}(0, y)\right]=q_{0}(y)[h]=0, \\
Z_{r}^{-, 0}(0, y)-Z_{r}^{+, 0}(0, y)=\left(\Pi_{-}+\Pi_{+}\right) q_{0}=0,
\end{gathered}
$$

where $[h]=h^{+}\left(v^{*}\right)-h^{-}\left(v^{*}\right)$.

Proof. Differentiating the representation of $w^{ \pm, 1}(\xi, y)$ at $\xi=0$,

$$
\begin{aligned}
u_{\xi}^{*}\left(s_{0}\right) w_{\xi}^{ \pm, 0}(0, y)= & {\left[\theta(y) u_{\xi}^{*}\left(s_{0}\right)-W^{ \pm, 0}(0, y)\right] u_{\xi \xi}^{*}\left(s_{0}\right) } \\
& -\int_{ \pm \infty}^{0}\left[f_{u}\left(u^{*}\left(\xi+s_{0}\right)\right) W^{ \pm, 0}(0, y)-q_{0}(y)\right] u_{\xi}^{*}\left(\xi+s_{0}\right) d \xi \\
= & \theta(y) u_{\xi}^{*}\left(s_{0}\right) u_{\xi \xi}^{*}\left(s_{0}\right)+q_{0}(y)\left(u^{*}\left(s_{0}\right)-h^{ \pm}\left(v^{*}\right)\right) .
\end{aligned}
$$

Here we used the fact that $u_{\xi \xi \xi}^{*}\left(s_{0}\right)+f_{u}\left(u^{*}\left(\xi+s_{0}\right)\right) u_{\xi}^{*}\left(\xi+s_{0}\right)=0$. Thus we obtain (3.20). (3.21) is obvious.

Lemma 3.3 implies that $q_{0}(y) \equiv 0$. Then we see that

$$
W^{ \pm, 0}(x) \equiv 0 \equiv Z^{ \pm, 0}(x)
$$

and $w^{ \pm, 0}$ is represented as

$$
w^{ \pm, 0}(\xi, y)=u_{\xi}^{*}\left(\xi+s_{0}(y)\right) \theta(y) .
$$

In the following, we omit the superscript \pm of $w^{ \pm, 0}(\xi, y)$.

Next we consider the $O(\epsilon)$-term of (3.19).

Lemma 3.4. $O(\epsilon)$-terms of (3.19) are equivalent to

$$
\begin{gathered}
u_{\xi}^{*}\left(s_{0}(y)\right)\left[W_{r}^{-, 0}(0, y)+w_{\xi}^{-, 1}(0, y)-W_{r}^{+, 0}(0, y)-w_{\xi}^{+, 1}(0, y)\right]=q_{1}(y)[h]=0, \\
Z_{r}^{-, 1}(0, y)-Z_{r}^{+, 1}(0, y)=\left(\Pi_{-}+\Pi_{+}\right) q_{1}=0 .
\end{gathered}
$$

Proof. In view of (3.22), the equation for $w^{ \pm, 1}$ is recast as

$$
\begin{aligned}
& w_{\xi \xi}^{ \pm, 1}+f_{u}\left(u^{*}\left(\xi+s_{0}\right)\right) w^{ \pm, 1} \\
& \quad+(N-1) H(0, y) w_{\xi}^{0}+f_{u}\left(u^{*}\left(\xi+s_{0}\right)\right) W^{ \pm, 1}(0, y)+\tilde{f}_{u}^{1} w_{\xi}^{0}-q_{1}(y)=0 .
\end{aligned}
$$

Differentiating the representation of $w^{ \pm, 1}(\xi, y)$ at $\xi=0$,

$$
\begin{aligned}
-u_{\xi}^{*}\left(s_{0}\right) w_{\xi}^{ \pm, 1}(0, y)= & \theta(y) \int_{ \pm \infty}^{0}\left[(N-1) H(0, y) u_{\xi \xi}^{*}\left(\xi+s_{0}\right)+\tilde{f}_{u}^{1} u_{\xi}^{*}\left(\xi+s_{0}\right)\right] \\
& \times u_{\xi}^{*}\left(\xi+s_{0}\right) d \xi-q_{1}(y) \int_{ \pm \infty}^{0} u_{\xi}^{*}\left(\xi+s_{0}\right) d \xi \\
= & \theta(y)\left[u_{\xi}^{ \pm, 1}(0, y) u_{\xi \xi}^{*}\left(s_{0}\right)-u_{\xi}^{*}\left(s_{0}\right) u_{\xi \xi}^{ \pm, 1}(0, y)\right] \\
& -q_{1}(y) \int_{ \pm \infty}^{0} u_{\xi}^{*}\left(\xi+s_{0}\right) d \xi
\end{aligned}
$$


Here we used the fact proved in Appendix D that

$$
\begin{aligned}
& \int_{ \pm \infty}^{0}\left[(N-1) H(0, y) u_{\xi \xi}^{*}\left(\xi+s_{0}\right)+\tilde{f}_{u}^{1} u_{\xi}^{*}\left(\xi+s_{0}\right)\right] u_{\xi}^{*}\left(\xi+s_{0}\right) d \xi \\
& \quad=u_{\xi}^{ \pm, 1}(0, y) u_{\xi \xi}^{*}\left(s_{0}\right)-u_{\xi}^{*}\left(s_{0}\right) u_{\xi \xi}^{ \pm, 1}(0, y) .
\end{aligned}
$$

By using the facts that $u_{\xi}^{-, 1}(0, y)=u_{\xi}^{+, 1}(0, y)$ and $u_{\xi \xi}^{-, 1}(0, y)=u_{\xi \xi}^{+, 1}(0, y)$, we have

$$
u_{\xi}^{*}\left(s_{0}(y)\right)\left[w_{\xi}^{-, 1}(0, y)-w_{\xi}^{+, 1}(0, y)\right]=q_{1}(y) \int_{-\infty}^{\infty} u_{\xi}^{*}(\xi) d \xi=q_{1}(y)[h] .
$$

Thus we obtain (3.23). Equation (3.24) is obvious.

Lemma 3.4 implies that $q_{1}(y) \equiv 0$, and then we see that

$$
W^{ \pm, 1}(x) \equiv 0 \equiv Z^{ \pm, 1}(x) .
$$

Let us define new functions $\tilde{\Phi}$ and $\tilde{\Psi}$ as follows:

$\tilde{\Phi}\left(\theta, q_{2}, \lambda_{1}, \epsilon\right):=\frac{1}{\epsilon^{2}} \Phi\left(\theta, 0,0, q_{2}, \lambda_{1}, \epsilon\right), \quad \tilde{\Psi}\left(\theta, q_{2}, \lambda_{1}, \epsilon\right):=\frac{1}{\epsilon^{2}} \Psi\left(\theta, 0,0, q_{2}, \lambda_{1}, \epsilon\right)$.

LEMMA 3.5. $\tilde{\Phi}\left(\theta, q_{2}, \lambda_{1}, 0\right)=0$ and $\tilde{\Psi}\left(\theta, q_{2}, \lambda_{1}, 0\right)=0$ are equivalent to

$$
\begin{gathered}
{\left[m^{2} \Delta^{\Gamma^{*}}+m^{2} H^{*}(y)-V_{r}^{1}(0, y) J^{\prime}\left(v^{*}\right)\right] \theta+J^{\prime}\left(v^{*}\right) q_{2}(y)=0,} \\
\left(\Pi_{-}+\Pi_{+}\right) q_{2}-\left(1+\lambda_{1}\right)[h] \theta=0 .
\end{gathered}
$$

Proof. See Appendix C.

Proof of Theorem 1.3. Using Lemma 3.5, we obtain (1.13).

4. Applications. In this section, we apply the results of the previous sections to the case where the domain $\Omega$ is a ball or a rectangle. In the following, we assume that the nonlinearity takes the form

$$
f(u)=u-u^{3} .
$$

First we note that the constants appeared in the previous section are computed as follows.

LEMMA 4.1.

$$
v^{*}=0, \quad h^{ \pm}\left(v^{*}\right)= \pm 1, \quad J^{\prime}\left(v^{*}\right)=-2, \quad[h]=2, \quad m^{2}=\frac{2 \sqrt{2}}{3}=\frac{4}{3 \sqrt{2}} .
$$

Proof. This is proved by straightforward computation.

4.1. Stability of radially symmetric solutions. In this subsection, we study the radially symmetric solution when $\Omega$ is a ball of radius $R$. It is convenient to introduce new coordinate system $x=(r, y)$, where $x=r y$ for $r \in[0, R]$ and $y \in \partial \Omega=$ $S^{N-1}$.

Then the rescaled reduced problem (1.7)-(1.9) can be rewritten as

$$
\begin{cases}V_{r r}^{+}+\frac{N-1}{r} V_{r}^{+}+G^{+}=0, & r_{0}<r<R, \\ V^{+}\left(r_{0}\right)=\frac{m^{2}(N-1)}{J^{\prime}\left(v^{*}\right) r_{0}}, & V_{r}^{+}(1)=0,\end{cases}
$$




$$
\begin{cases}V_{r r}^{-}+\frac{N-1}{r} V_{r}^{-}+G^{-}=0, & 0<r<r_{0}, \\ V_{r}^{-}(0)=0, & V^{-}\left(r_{0}\right)=\frac{m^{2}(N-1)}{J^{\prime}\left(v^{*}\right) r_{0}},\end{cases}
$$

and

$$
V_{r}^{-}\left(r_{0}\right)=V_{r}^{+}\left(r_{0}\right)
$$

where $G^{ \pm}:= \pm 1-\bar{u}$. Here $V^{-}(r, y), V^{+}(r, y)$ and $r_{0}$ are unknown functions and a parameter, respectively. The solutions of $(4.1)_{ \pm}$have the following expressions:

$$
\begin{aligned}
& V^{+}(r)=\frac{m^{2}(N-1)}{J^{\prime}\left(v^{*}\right) r_{0}}+\frac{G^{+}}{N} \int_{r_{0}}^{r}\left(R^{N} t^{1-N}-t\right) d t, \\
& V^{-}(r)=\frac{m^{2}(N-1)}{J^{\prime}\left(v^{*}\right) r_{0}}+\frac{G^{-}}{2 N}\left(r_{0}^{2}-r^{2}\right) .
\end{aligned}
$$

Then, by using the condition (4.2), $r_{0}$ is uniquely determined as

$$
r_{0}=\left(\frac{G^{+}}{[h]}\right)^{1 / N} R,
$$

and the interface $\Gamma^{*}$ is defined by

$$
\Gamma^{*}=\left\{x \in \mathbf{R}^{N}|| x \mid=r_{0}\right\} .
$$

The following existence theorem can be proved in a similar way as in [5], so we omit it (see also [19]).

THEOREM 4.2. There exists a constant $\epsilon_{0}>0$ such that (1.5) and (1.6) have an $\epsilon$-family of radially symmetric layer solutions $\left(u^{\epsilon}(r), v^{\epsilon}(r)\right)$ for $\epsilon \in\left(0, \epsilon_{0}\right]$ satisfying the following:

(i) $\lim _{\epsilon \rightarrow 0} v^{\epsilon}=v^{*}$ uniformly on $\Omega$.

(ii) For each $\delta>0$,

$$
\lim _{\epsilon \rightarrow 0} u^{\epsilon}(r)= \begin{cases}-1, & 0 \leq r \leq r_{0}-\delta, \\ 1, & r_{0}+\delta \leq r \leq R .\end{cases}
$$

The asymptotic form of $\left(U^{\epsilon}(r), v^{\epsilon}(r)\right)$ is given in section 2 .

Using Lemma 4.1 and the above results, we can find that the operator $L$ is recast as

$$
L=\frac{1}{r_{0}^{2}} \Delta^{S}+\frac{N-1}{r_{0}^{2}}+\frac{3 \sqrt{2}}{2} \frac{1+\bar{u}}{N} r_{0}-3 \sqrt{2} \mathcal{T}(\cdot)
$$

We prepare two lemmas before we state the key proposition.

Lemma 4.3. All the eigenvalues of $\mathcal{T}$ and $L$ are real. More precisely,

(i) the $j$ th eigenvalue $\Lambda_{j}$ of $\mathcal{T}$ is given by

$$
\Lambda_{j}=\alpha R \hat{\Lambda}(j, \alpha)
$$

where

$$
\hat{\Lambda}(z, \alpha)=\frac{(z+N-2) \alpha^{2 z+N-2}+z}{z(2 z+N-2)}, r_{0}=\alpha R, \alpha=\alpha(\bar{u})=\left(\frac{1-\bar{u}}{2}\right)^{1 / N} .
$$

Then the associated eigenfunction $\beta_{j}^{m}(y)$ is the harmonic function of degree $m$. 
(ii) The $j$ th eigenvalue $L$ is real and given by $\Sigma_{j}=\Sigma(j, \alpha, R)$, where

$$
\Sigma(z, \alpha, R)=\frac{1}{\alpha^{2} R^{2}}\left[-z^{2}-(N-1) z+N-1\right]+3 \sqrt{2}\left[\frac{1-\alpha^{N}}{N}-\hat{\Lambda}(z, \alpha)\right] \alpha R .
$$

Then the associated eigenfunction is the same as that of $\mathcal{T}$.

Proof. See Appendix E.

Lemma 4.4 (properties of $\hat{\Lambda}(z, \alpha)$ and $\Sigma(z, \alpha, R)$ ).

(i) For $z \geq 1$ and $\alpha \in(0,1)$,

$$
\frac{\partial}{\partial z} \hat{\Lambda}(z, \alpha)<0, \quad \frac{\partial^{2}}{\partial z^{2}} \hat{\Lambda}(z, \alpha)>0 .
$$

(ii)

$$
\begin{aligned}
\frac{\partial}{\partial z} \Sigma(1, \alpha, R)= & -\frac{N}{\alpha^{2} R^{2}} \\
& -\frac{3 \sqrt{2} \alpha R}{N^{2}}\left[-\alpha^{N}\left\{2+4(N-2)+(N-2)^{2}\right\}+2(N-1) N \alpha^{N} \log \alpha-2\right], \\
\Sigma(1, \alpha, R)= & -3 \sqrt{2} \alpha^{N+1} R<0 \quad \text { for } \alpha \in(0,1), \\
& \frac{\partial^{2}}{\partial z^{2}} \Sigma(z, \alpha, R)<0 \quad \text { for } z \geq 1 \quad \text { and } \quad \alpha \in(0,1) .
\end{aligned}
$$

Proof. These results can be obtained by direct calculations.

The following proposition is a key to prove Theorem 1.4.

PROPOSITION 4.5.

(i) The $j$ th principal eigenvalue $\lambda_{j}^{*}$ of (1.13) is given by the following form.

$$
\lambda_{j}^{*}=\lambda_{j}^{*}(\alpha, \bar{u})=\frac{1}{3 \sqrt{2}} \cdot \frac{\Sigma(j, \alpha(\bar{u}), R)}{\alpha R \hat{\Lambda}(j, \alpha(\bar{u}), R)} .
$$

Moreover, it holds that

(ii) for any fixed $R \in(0, \infty)$, there exists $\bar{u}_{0}=\bar{u}_{0}(R) \in(-1,1)$ such that

$$
\Sigma(z, \alpha(\bar{u}), R)<0 \quad \text { for } \quad \bar{u} \in\left(0, \bar{u}_{0}\right) \quad \text { and } \quad z \geq 1 .
$$

(iii) For any fixed $\bar{u} \in(-1,1)$, there exist $R_{0}=R_{0}(\bar{u})>0$ and integer $z_{0} \geq 1$ such that

$$
\Sigma\left(z_{0}, \alpha(\bar{u}), R_{0}\right)>0 .
$$

Proof. (i) Noting that $L$ and $\mathcal{T}$ have the same eigenfunctions $\left\{\beta_{j}(y)\right\}_{j=1}^{\infty}$, we can rewrite (1.13) as

$$
L \beta_{j}=3 \sqrt{2} \lambda^{*} \mathcal{T}\left(\beta_{j}\right)
$$

which leads to (4.6).

(ii) Note that $\alpha(\bar{u})$ is a monotone decreasing function of $\bar{u}$. Since $\frac{\partial}{\partial z} \Sigma(1, \alpha, R)<0$ and $\frac{\partial^{2}}{\partial z^{2}} \Sigma(z, \alpha, R)<0$ for sufficiently small $\alpha$, we obtain (ii).

(iii) For fixed $\alpha$, we choose $z_{0}$ satisfying $\frac{1-\alpha^{N}}{N}-\hat{\Lambda}\left(z_{0}, \alpha\right)>0$. Then the sign of the first term of (4.5) is negative and that of the second one is positive. Therefore, for sufficiently large $R$, it holds that $\Sigma\left(z_{0}, \alpha, R\right)>0$. 
Proof of Theorem 1.4. It is a direct consequence of Proposition 4.5.

4.2. Stability of planar solutions. Let $\Omega$ be a rectangle in $(x, y)$-plane $\Omega:=$ $(0, X) \times(0, Y)$. Then the rescaled reduced problem (1.7)-(1.9) is recast as

$$
\begin{aligned}
& \begin{cases}V_{x x}^{+}+G^{+}=0, & x_{0}<x<X, \\
V^{+}\left(x_{0}\right)=0, & V_{x}^{+}(X)=0,\end{cases} \\
& \begin{cases}V_{x x}^{-}+G^{-}=0, & 0<x<x_{0}, \\
V_{x}^{-}(0)=0, & V^{-}\left(x_{0}\right)=0,\end{cases}
\end{aligned}
$$

and

$$
V_{x}^{-}\left(x_{0}\right)=V_{x}^{+}\left(x_{0}\right)
$$

where $G^{ \pm}:= \pm 1-\bar{u}, \Omega_{+}\left(\Gamma^{*}\right)=\left\{(x, y) \in \mathbf{R}^{2} \mid x_{0}<x<X, 0<y<Y\right\}, \Omega_{-}\left(\Gamma^{*}\right)=$ $\left\{(x, y) \in \mathbf{R}^{2} \mid 0<x<x_{0}, 0<y<Y\right\}$, and $\Gamma^{*}=\left\{\left(x_{0}, y\right) \in \mathbf{R}^{2} \mid 0<y<Y\right\}$. Here we used the fact that $H_{1}(y)=0$. We can easily show that (4.7) - and $(4.7)_{+}$have unique solutions given by

$$
\begin{aligned}
& V^{-}(x)=-\frac{1}{2} G^{-}\left[x^{2}-x_{0}^{2}\right], \\
& V^{+}(x)=-G^{+}\left[\frac{1}{2}\left(x^{2}-x_{0}^{2}\right)-X\left(x-x_{0}\right)\right] .
\end{aligned}
$$

Then, by using the $C^{1}$-matching condition (4.8), we can uniquely determine $x_{0}$ as

$$
x_{0}=\frac{G^{+} X}{[h]},
$$

so the derivative $V_{x}\left(x_{0}\right)$ is given by

$$
V_{x}\left(x_{0}\right)=-\frac{G^{+} G^{-} X}{[h]}=\frac{1}{2} X(1-\bar{u})(1+\bar{u}) .
$$

The existence results of the planar solution to (1.5) and (1.6) is given by Taniguchi and Nishiura [23].

THEOREM 4.6 (see [23]). There exists a constant $\epsilon_{0}>0$ such that (1.5) and (1.6) have an $\epsilon$-family of stationary planar solutions $\left(u^{\epsilon}(x), v^{\epsilon}(x)\right)$ independent of $y \in[0, Y]$ for $\epsilon \in\left(0, \epsilon_{0}\right]$ satisfying the following:

(i) $\lim _{\epsilon \rightarrow 0} v^{\epsilon}=v^{*}$ uniformly on $\Omega$.

(ii) For each $\delta>0$,

$$
\lim _{\epsilon \rightarrow 0} u^{\epsilon}(x)= \begin{cases}-1, & 0 \leq x \leq x_{0}-\delta, \\ 1, & x_{0}+\delta \leq x \leq X .\end{cases}
$$

Using Lemma 4.1 and the above results, we can find that the operator $L$ is recast as

$$
L=\frac{d^{2}}{d y^{2}}-\frac{3 \sqrt{2}}{2}\left[\frac{1}{2} X(\bar{u}-1)(\bar{u}+1)+2 \mathcal{T}(\cdot)\right] .
$$


The spectral properties of $L$ and $\mathcal{T}$ are given in the following two lemmas.

Lemma 4.7. All the eigenvalues of $\mathcal{T}$ and $L$ are real. More precisely,

(i) the $j$ th eigenvalue $\Lambda_{j}$ of $\mathcal{T}$ is given by

$$
\Lambda_{j}=X \Lambda(\kappa \pi j, \bar{u})
$$

where

$$
\Lambda(z, \bar{u}):=\frac{\cosh z+\cosh \bar{u} z}{2 z \sinh z}
$$

and $\kappa:=X / Y$. Then the associated eigenfunction is $\beta_{j}(y)=\cos \left(\tau_{j} y\right)$, where $\tau_{j}=$ $\pi j / Y$.

(ii) The $j$ th eigenvalue of $L$ is real and given by $\Sigma_{j}=\Sigma(\kappa \pi j, X, \bar{u})$, where

$$
\Sigma(z, X, \bar{u}):=-\left(\frac{z}{X}\right)^{2}+\frac{3 \sqrt{2}}{2} X\left[\frac{1}{2}(1-\bar{u})(1+\bar{u})-2 \Lambda(z, \bar{u})\right] .
$$

Then the associated eigenfunction is the same as that of $\mathcal{T}$.

Proof. See Appendix F.

Lemma 4.8 (properties of $\Lambda(z, \bar{u})$ ). For $\bar{u} \in(-1,1)$ and $z>0$,

$$
\begin{aligned}
& \Lambda(z, \bar{u})>0, \quad \frac{\partial}{\partial z} \Lambda(z, \bar{u})<0, \quad \frac{\partial^{2}}{\partial z^{2}} \Lambda(z, \bar{u})>0, \\
& \lim _{z \rightarrow+0} \Lambda(z, \bar{u})=\infty, \quad \lim _{z \rightarrow \infty} z \Lambda(z, \bar{u})=\frac{1}{2} .
\end{aligned}
$$

Proof. These results can be obtained by direct calculations.

Using the above two lemmas, we can prove the following proposition.

Proposition 4.9. (i) The $j$ th principal eigenvalue $\lambda_{j}^{*}$ of (1.13) is given by the following form:

$$
\lambda_{j}^{*}=\lambda_{j}^{*}(\kappa, X, \bar{u})=\frac{1}{3 \sqrt{2}} \cdot \frac{\Sigma(\kappa \pi j, X, \bar{u})}{X \Lambda(\kappa \pi j, \bar{u})} .
$$

(ii) The nullcline of $\Sigma(z, X, \bar{u})$ as a function of $z>0$ and $X>0$ is given by $\left\{(z, X(z, \bar{u})) \mid z>z_{0}\right\}$, where $z_{0}$ is a unique zero of $\frac{1}{2}\left(1-\bar{u}^{2}\right)-2 \Lambda(z, \bar{u})=0$ and

$$
X(z ; \bar{u})=\left(\frac{\sqrt{2}}{3}\right)^{1 / 3} z^{2 / 3}\left[\frac{1}{2}\left(1-\bar{u}^{2}\right)-2 \Lambda(z, \bar{u})\right]^{-1 / 3} .
$$

Moreover, $X(z ; \bar{u})$ has the following properties:

$$
\begin{gathered}
\lim _{z \rightarrow z_{0}+0} X(z ; \bar{u})=\infty, \\
\lim _{z \rightarrow \infty}\left[X(z ; \bar{u})-\left(\frac{2 \sqrt{2}}{3}\right)^{1 / 3}\left(1-\bar{u}^{2}\right)^{-1 / 3} z^{2 / 3}\right]=0, \\
\frac{d X}{d z}\left\{\begin{array}{lll}
<0 & \text { for } & z_{0}<z<z_{1}, \\
=0 & \text { for } & z=z_{1}, \\
>0 & \text { for } & z_{1}<z,
\end{array}\right.
\end{gathered}
$$

for some $z_{1}=z_{1}(\bar{u})$. 
Proof. (i) Noting that $L$ and $\mathcal{T}$ have the same eigenfunctions $\left\{\beta_{j}(y)\right\}_{j=1}^{\infty}$, we can rewrite (1.13) as

$$
L \beta_{j}=3 \sqrt{2} \lambda^{*} \mathcal{T}\left(\beta_{j}\right)
$$

which leads to (4.11).

(ii) We define a function $g$ of $z$ and $X$ on $(0, \infty) \times(0, \infty)$ by

$$
g(z, X, \bar{u}):=X^{2} \Sigma(z, X, \bar{u})=-z^{2}+\frac{3 \sqrt{2}}{2} X^{3}\left[\frac{1}{2}\left(1-\bar{u}^{2}\right)-\Lambda(z, \bar{u})\right] .
$$

Using Lemma 4.8 , we see that $p(z, \bar{u}):=\frac{1}{2}\left(1-\bar{u}^{2}\right)-\Lambda(z, \bar{u})$ has a unique zero $z=z_{0}(\bar{u})$ and $p(z, \bar{u})>0$ for $z>z_{0}$. Then we can solve $g(z, X, \bar{u})=0$ in $X$ as (4.12). Noting the properties of $\Lambda(z, \bar{u})$, we obtain (4.13) and (4.14).

By using the implicit function theorem, we see that

$$
\frac{d X}{d z}=-\frac{\partial g}{\partial z} / \frac{\partial g}{\partial X}
$$

and $\frac{\partial g}{\partial X}=\frac{9 \sqrt{2}}{2} X^{2}\left[\left(1-\bar{u}^{2}\right) / 2-\Lambda(z, \bar{u})\right]>0$. Noting that $\frac{\partial g}{\partial z}=-2 z-3 \sqrt{2} X^{3} \Lambda_{z},(4.13)$ and $(4.14)$, we have

$$
\lim _{z \rightarrow z_{0}+0} \frac{\partial g}{\partial z}(z, X(z ; \bar{u}) ; \bar{u})=\infty, \quad \lim _{z \rightarrow \infty} \frac{\partial g}{\partial z}(z, X(z ; \bar{u}) ; \bar{u})=-\infty .
$$

Combining the above facts and $\frac{\partial^{2} g}{\partial z^{2}}=-2-3 \sqrt{2} X^{3} \Lambda_{z z}<0$, we can see that $\frac{\partial g}{\partial z}$ has a unique zero $z=z_{1}$ and $\frac{d X}{d z}$ satisfies (4.15).

Proof of Theorem 1.5. The proof follows from Proposition 4.9.

4.3. Justification of the SLEP equation for the planar case. In subsection 3.3 , we derived the SLEP equation for the principal parts of the critical eigenvalues and eigenfunctions. Since our method is constructive, it is not a priori clear that there are no other dangerous eigenvalues. But, fortunately, when the domain $\Omega$ is a ball or a rectangle, we can justify our results. Precisely speaking, the principal parts of all dangerous eigenvalues are reduced to the solutions to (1.13).

In this subsection, we outline the justification of (1.13) when $\Omega$ is a rectangle $(0, X) \times(0, Y)$. When $\Omega$ is a ball, we can justify (1.13) in a parallel way. See [19] and $[20]$ for the activator-inhibitor case.

By using a complete orthonormal system $\left\{\Phi_{j}(y)\right\}_{j=0}^{\infty}$ in $L^{2}(0, Y)$, where

$$
\Phi_{j}(y)= \begin{cases}1 / \sqrt{Y}, & j=0, \\ \sqrt{2 / Y} \cos (\pi j y / Y), & j>0,\end{cases}
$$

$(w, z)$ is expanded as

$$
w(x, y)=\sum_{j=0}^{\infty} w_{j}(x) \Phi_{j}(y), \quad z(x, y)=\sum_{j=0}^{\infty} z_{j}(x) \Phi_{j}(y),
$$

where

$$
w_{j}(x)=\int_{0}^{Y} w(x, y) \Phi_{j}(y) d y, z_{j}(x)=\int_{0}^{Y} z(x, y) \Phi_{j}(y) d y
$$


in $L^{2}(\Omega)$. By using the above notation, the eigenvalue problem (3.1) is rewritten as

$$
\left\{\begin{array}{l}
0=L^{\epsilon, j} w_{j}-z_{j}, \\
0=M^{j} z_{j}+\epsilon w_{j}+\lambda w_{j},
\end{array} \quad x \in I:=(0, X),\right.
$$

with the boundary condition

$$
\frac{d w_{j}}{d x}(0)=0=\frac{d w_{j}}{d x}(X), \frac{d z_{j}}{d x}(0)=0=\frac{d z_{j}}{d x}(X)
$$

for $j=0,1, \ldots$, where

$$
L^{\epsilon, j}:=\epsilon^{2} \frac{d^{2}}{d x^{2}}+f_{u}^{\epsilon}-\epsilon^{2} \mu_{j}, \quad M^{j}:=\frac{d^{2}}{d x^{2}}-\mu_{j}, \quad \mu_{j}=\left(\frac{\pi j}{Y}\right)^{2} .
$$

Then the condition $\iint_{\Omega} w d x d y=0$ is equivalent to either

$$
\int_{0}^{X} w_{0}(x) d x=0 \quad\left(w_{0}(x) \not \equiv 0\right)
$$

or

$$
w_{0}(x) \equiv 0
$$

since $\int_{0}^{Y} \Phi_{j}(y) d y=0$ for $j=1,2, \ldots$.

First, we consider the former case. Let $\left\{\phi_{k}^{\epsilon, j}\right\}_{k \geq 0}$ be the complete orthonormal set in $L^{2}(I)$ consisting of the eigenfunctions of $L^{\epsilon, j}$, and $\left\{\zeta_{k}^{\epsilon, j}\right\}_{k \geq 0}$ the associated eigenvalues. They have the following properties.

Lemma 4.10 (Nishiura [10] and [11]).

(i) It holds that

$$
\zeta_{0}^{\epsilon, j}>0>-\delta>\zeta_{1}^{\epsilon, j}>\zeta_{2}^{\epsilon, j}>\cdots
$$

for sufficiently small $\epsilon>0$, where $\delta$ is a positive constant independent of $j \geq 0$ and $\epsilon>0$.

(ii) $\lim _{\epsilon \downarrow 0} \frac{\zeta_{0}^{\epsilon, j}}{\epsilon^{2}}=\hat{\zeta}_{0}^{*}-\mu_{j}$, where $\hat{\zeta}_{0}^{*}=-\frac{1}{m^{2}} J^{\prime}\left(v^{*}\right) V_{x}\left(x_{0}\right)$.

(iii) $\lim _{\epsilon \downarrow 0} \frac{\phi_{0}^{\epsilon, j}}{\sqrt{\epsilon}}=\frac{[h]}{m} \delta_{x_{0}}$ in $H^{-1}(I)$-sense, where $\delta_{x_{0}}$ is a Dirac's $\delta$-function at $x=x_{0}$.

We decompose $w$ as

$$
w=\left(L^{\epsilon, j}\right)^{-1} z=\frac{\left\langle z, \phi_{0}^{\epsilon, j}\right\rangle}{\zeta_{0}^{\epsilon, j}} \phi_{0}^{\epsilon, j}+\left(L^{\epsilon, j}\right)^{\dagger}(z)
$$

where

$$
\left(L^{\epsilon, j}\right)^{\dagger}:=\sum_{n \geq 1} \frac{\left\langle\cdot, \phi_{n}^{\epsilon, j}\right\rangle}{\zeta_{n}^{\epsilon, j}} \phi_{n}^{\epsilon, j}
$$

$\left(L^{\epsilon, j}\right)^{\dagger}$ has the following properties. 
Lemma 4.11 (Nishiura [10] and [11]). There exists a constant $\epsilon_{0}>0$ such that $\left(L^{\epsilon, j}\right)^{\dagger}$ is a uniformly $L^{2}$-bounded operator for $\epsilon \in\left(0, \epsilon_{0}\right)$ and $j \geq 0$. Moreover, the following property holds:

$$
\lim _{\epsilon \rightarrow 0}\left(L^{\epsilon, j}\right)^{\dagger} p=\frac{p}{f_{u}^{*}} \text { strongly in } L^{2}-\text { sense, }
$$

where $p \in L^{2}(I) \cap L^{\infty}(I)$ and $f_{u}^{*}:=f_{u}\left(h^{ \pm}\left(v^{*}\right)\right)$.

The eigenvalue problem (4.16) and the condition $\iint_{\Omega} w d x d y=0$ with $j=0$ are recast as

$$
z_{x x}+(\epsilon+\lambda)\left[\frac{\left\langle z, \phi_{0}^{\epsilon, 0}\right\rangle}{\zeta_{0}^{\epsilon, 0}} \phi_{0}^{\epsilon, 0}+\left(L^{\epsilon, 0}\right)^{\dagger}(z)\right]=0
$$

and

$$
\frac{\left\langle z, \phi_{0}^{\epsilon, 0} / \sqrt{\epsilon}\right\rangle}{\epsilon} \int_{0}^{X} \frac{1}{\zeta_{0}^{\epsilon} / \epsilon^{2}} \frac{\phi_{0}^{\epsilon, 0}}{\sqrt{\epsilon}} d x+\int_{0}^{X}\left(L^{\epsilon, 0}\right)^{\dagger}(z) d x=0 .
$$

In view of Lemmas 4.10 and 4.11 , we see that

$$
\lim _{\epsilon \rightarrow 0} \int_{0}^{X} \frac{1}{\zeta_{0}^{\epsilon} / \epsilon^{2}} \frac{\phi_{0}^{\epsilon, 0}}{\sqrt{\epsilon}} d x=\frac{[h]}{m \hat{\zeta}_{0}^{*}}
$$

and $\lim _{\epsilon \rightarrow 0} \int_{0}^{X}\left(L^{\epsilon, 0}\right)^{\dagger}(z) d x$ is bounded. Consequently, the following limits exist:

$$
\hat{z}^{*}:=\frac{m}{[h]} \lim _{\epsilon \downarrow 0} \frac{1}{\epsilon}\left\langle z, \phi_{0}^{\epsilon, 0} / \sqrt{\epsilon}\right\rangle, \quad 0=\lim _{\epsilon \downarrow 0}\left\langle z, \phi_{0}^{\epsilon, 0} / \sqrt{\epsilon}\right\rangle .
$$

Now we rewrite (4.17) in a weak form

$$
\begin{gathered}
-\left\langle z_{x}, \psi_{x}\right\rangle+(\epsilon+\lambda)\left[\frac{\left\langle z, \phi_{0}^{\epsilon, 0} / \sqrt{\epsilon}\right\rangle / \epsilon}{\zeta_{0}^{\epsilon} / \epsilon^{2}}\left\langle\phi_{0}^{\epsilon, 0} / \sqrt{\epsilon}, \psi\right\rangle+\left\langle\left(L^{\epsilon, 0}\right)^{\dagger}(z), \psi\right\rangle\right]=0, \\
z \in H_{N}^{1}(I), \psi \in H^{1}(I),
\end{gathered}
$$

where $H_{N}^{1}(I)$ is the space of closure of $\{\cos (n \pi x / X)\}_{n=0}^{\infty}$ in $H^{1}(I)$. Then the limit function $z^{*}=\lim _{\epsilon \rightarrow 0} z$ must exist and satisfy the following limit equation:

$$
-\left\langle z_{x}^{*}, \psi_{x}\right\rangle+\lambda\left[\frac{[h]^{2} \hat{z}^{*}}{m^{2} \hat{\zeta}_{0}^{*}}\left\langle\delta_{x_{0}}, \psi\right\rangle+\frac{1}{f_{u}^{*}}\left\langle z^{*}, \psi\right\rangle\right]=0, \quad z^{*}\left(x_{0}\right)=0 .
$$

This is equivalent to the next system:

$$
\begin{aligned}
& \begin{cases}Z_{x x}^{-}+\frac{\lambda}{f_{u}^{*}} Z^{-}=0, & x \in\left(0, x_{0}\right), \\
Z_{x}^{-}(0)=0, & Z^{-}\left(x_{0}\right)=0,\end{cases} \\
& \begin{cases}Z_{x x}^{+}+\frac{\lambda}{f_{u}^{*}} Z^{+}=0, & x \in\left(x_{0}, X\right), \\
Z^{+}\left(x_{0}\right)=0, & Z_{x}^{+}(X)=0,\end{cases}
\end{aligned}
$$




$$
Z_{x}^{+}\left(x_{0}\right)-Z_{x}^{-}\left(x_{0}\right)=-\lambda \frac{[h]^{2} \hat{z}^{*}}{m^{2} \hat{\zeta}_{0}^{*}}
$$

Lemma 4.12. There exists a constant $\delta>0$ such that (4.18)-(4.20) have no nontrivial solutions for $\lambda>-\delta$ for the case (i), i.e., $\int_{0}^{X} w_{0}(x) d x=0\left(w_{0}(x) \not \equiv 0\right)$.

Proof. When $\lambda=0,(4.18)-(4.20)$ have a unique solution $Z^{-}(x) \equiv 0$ and $Z^{+}(x) \equiv 0$.

If $\lambda>0$, the general solution $Z(x)$ of (4.18) is represented as

$$
Z(x)=A \exp (\alpha x)+B \exp (-\alpha x)
$$

where $\alpha^{2}=-\lambda / f_{u}^{*}, \alpha>0$. Then, by using the boundary conditions, we obtain $A=0$ and $B=0$. Similarly, we can prove that (4.19) has no nonhomogeneous solutions.

If $-\delta<\lambda<0$, where $-\delta:=\frac{\pi^{2}}{X^{2}} f_{u}^{*}$, the general solutions $Z^{-}(x)=C^{-} \cos \beta x$ and $Z^{+}(x)=C^{+} \cos \beta(X-x)$, where $\beta^{2}=\lambda / f_{u}^{*}$ and $\beta>0$, cannot satisfy the boundary conditions $Z^{-}\left(x_{0}\right)=0$ and $Z^{+}\left(x_{0}\right)=0$ simultaneously.

Next, we consider the case (ii) $w_{0}(x) \equiv 0$. The eigenvalue problem (4.16) with $j>0$ is recast as

$$
-\left\langle z_{x}, \psi_{x}\right\rangle-\mu_{j}\langle z, \psi\rangle+\frac{\epsilon+\lambda}{\epsilon}\left[\frac{\left\langle z, \phi_{0}^{\epsilon, j} / \sqrt{\epsilon}\right\rangle}{\zeta_{0}^{\epsilon, j} / \epsilon^{2}}\left\langle\phi_{0}^{\epsilon, j} / \sqrt{\epsilon}, \psi\right\rangle+\epsilon\left\langle\left(L^{\epsilon, j}\right)^{\dagger}(z), \psi\right\rangle\right]=0,
$$

where $z \in H_{N}^{1}(I), \psi \in H^{1}(I)$.

By Lemmas 4.10 and 4.11, we see that $\lambda=O(\epsilon)$ and the limit function $z^{*}=$ $\lim _{\epsilon \rightarrow 0} z$ must exist. Then $z^{*}$ satisfies the following limit equation:

$$
-\left\langle z_{x}^{*}, \psi_{x}\right\rangle-\mu_{j}\left\langle z^{*}, \psi\right\rangle+(1+\hat{\lambda}) \frac{[h]^{2}}{m^{2}} \frac{\left\langle z^{*}, \delta_{x_{0}}\right\rangle}{\hat{\zeta}_{0}^{*}-\mu_{j}}\left\langle\delta_{x_{0}}, \psi\right\rangle=0,
$$

where $\hat{\lambda}=\lim _{\epsilon \rightarrow 0} \lambda / \epsilon$. Hereafter, we normalize the limit eigenfunction $z^{*}$ as $\left\langle z^{*}, \delta_{0}\right\rangle=1$. Then (4.21) is equivalent to the next system:

$$
\left\{\begin{array}{l}
z_{x x}^{*}-\mu_{j} z^{*}=0, \quad x \in\left(0, x_{0}\right) \cup\left(x_{0}, X\right) \\
\lim _{x \rightarrow x_{0}+0} z_{x}^{*}(x)-\lim _{x \rightarrow x_{0}-0} z_{x}^{*}(x)=-(1+\hat{\lambda}) \frac{[h]^{2}}{m^{2}\left(\hat{\zeta}_{0}^{*}-\mu_{j}\right)} \\
z^{*}\left(x_{0}\right)=1, \quad z_{x}^{*}(0)=0=z_{x}^{*}(X) .
\end{array}\right.
$$

Proposition 4.13. The eigenvalue $\hat{\lambda}$ of (4.22) is given by (4.11) for the case (ii), i.e., $w_{0}(x) \not \equiv 0$.

Proof. Note that $z^{*}$ is determined by the first equation with the third and fourth condition of (4.22). In fact, after simple computations, we have

$$
z^{*}(x)= \begin{cases}\frac{\cosh \tau_{j} x}{\cosh \tau_{j} x_{0}}, & 0<x<x_{0}, \\ \frac{\cosh \tau_{j}(x-X)}{\cosh \tau_{j}\left(X-x_{0}\right)}, & x_{0}<x<X .\end{cases}
$$


Here we used the facts that $\mu_{j}=\tau_{j}^{2}=(\pi j / Y)^{2}$. Then

$$
\begin{aligned}
\lim _{x \rightarrow x_{0}+0} z^{*}(x)-\lim _{x \rightarrow x_{0}-0} z^{*}(x) & =\frac{\tau \sinh \tau_{j}(x-X)}{\cosh \tau_{j}\left(X-x_{0}\right)}-\frac{\tau \sinh \tau_{j} x}{\cosh \tau_{j} x_{0}} \\
& =-\frac{1}{X} \frac{2 \tau_{j} X \sinh \tau_{j} X}{\cosh \tau_{j} X+\cosh \bar{u} \tau_{j} X} \\
& =-\frac{1}{X \Lambda(\kappa \pi j, \bar{u})} .
\end{aligned}
$$

Solving the second equation of (4.22) in $\hat{\lambda}$, we have

$$
\hat{\lambda}=\frac{m^{2}}{[h]^{2} X \Lambda(\kappa \pi j, \bar{u})}\left[-\mu_{j}+\hat{\zeta}_{0}^{*}-\frac{[h]^{2}}{m^{2}} X \Lambda(\kappa \pi j, \bar{u})\right] .
$$

Noting the facts that

$$
\kappa=\frac{X}{Y}, \quad \mu_{j}=\left(\frac{\kappa \pi j}{X}\right)^{2}, \quad \hat{\zeta}_{0}^{*}=\frac{3 \sqrt{2}}{4} X(1-\bar{u})(1+\bar{u}), \quad \frac{[h]^{2}}{m^{2}}=3 \sqrt{2},
$$

we obtain (4.11). Thus we complete the proof.

In view of Lemma 4.12 and Proposition 4.13, it is clear that all dangerous eigenvalues to stability are controlled by the SLEP equation (1.13) (or, equivalently, (4.22)).

5. Concluding remarks - activator-inhibitor case. In the preceding sections, we have discussed the stability of mesoscopic patterns in diblock copolymers. Here we show that our approach also works well for the activator-inhibitor system (1.4) under the Neumann boundary condition. We assume the following for the nonlinearity $g(u, v)$ :

$$
\pm g\left(h^{ \pm}\left(v^{*}\right), v^{*}\right)>0, \quad g_{u}\left(h^{ \pm}\left(v^{*}\right), v^{*}\right)>0,\left.\quad \frac{d}{d v} g\left(h^{ \pm}(v), v\right)\right|_{v=v^{*}}<0 .
$$

In the following, we will use the same notation defined in the previous sections without explanation. The reduced problem to (1.4) is given by

$$
\begin{gathered}
\begin{cases}D \Delta V^{-}=-g\left(h^{-}\left(v^{*}\right), v^{*}\right) & \text { in } \Omega_{-}(\Gamma), \\
V^{-}=-\frac{m^{2}(N-1)}{J^{\prime}\left(v^{*}\right)} H(0, y) & \text { on } \Gamma, \\
\frac{\partial V^{-}}{\partial n}=0 & \text { on } \partial \Omega,\end{cases} \\
\begin{cases}D \Delta V^{+}=-g\left(h^{+}\left(v^{*}\right), v^{*}\right) & \text { in } \Omega_{+}(\Gamma), \\
V^{+}=-\frac{m^{2}(N-1)}{J^{\prime}\left(v^{*}\right)} H(0, y) & \text { on } \Gamma,\end{cases} \\
\frac{\partial V^{+}}{\partial \nu}=\frac{\partial V^{-}}{\partial \nu} \quad \text { on } \Gamma .
\end{gathered}
$$

Concerning the existence of stationary solutions, we have the following corollary.

Corollary of Theorem 1.2. Assume that (A1)-(A3) in section 1, (A7), and the following $\left(\mathrm{A} 4^{\prime}\right)-\left(\mathrm{A} 5^{\prime}\right)$ are satisfied: 
$\left(\mathrm{A} 4^{\prime}\right)$ There exists a solution $\left(V^{*}, \Gamma^{*}\right) \in C^{1}(\bar{\Omega}) \times \mathcal{F}$ of $(5.1)-(5.3)$, where

$$
V^{*}(x)= \begin{cases}V^{-}(x), & x \in \Omega_{-}\left(\Gamma^{*}\right), \\ V^{+}(x), & x \in \Omega_{+}\left(\Gamma^{*}\right) .\end{cases}
$$

$V^{ \pm}$are smooth solutions of (5.1) and (5.2), respectively, satisfying (5.3).

$\left(\mathrm{A} 5^{\prime}\right)$ There is a bounded linear operator $L^{\dagger}:(I-P) C^{\alpha}\left(\Gamma^{*}\right) \rightarrow(I-P) C^{2, \alpha}\left(\Gamma^{*}\right)$ called the inverse of the operator

$$
L:=\Delta^{\Gamma^{*}}+H^{*}(y)-\frac{1}{m^{2}} J^{\prime}\left(v^{*}\right) V_{r}^{*}(0, y)+\frac{1}{D m^{2}}[g] J^{\prime}\left(v^{*}\right) \mathcal{T}(\cdot)
$$

such that $L L^{\dagger}=I$ on $(I-P) C^{\alpha}\left(\Gamma^{*}\right)$ and $L^{\dagger} L=I-P$ on $(I-P) C^{2, \alpha}\left(\Gamma^{*}\right)$. Here, $\Delta^{\Gamma^{*}}$ is the Laplace-Beltrami operator on $\Gamma^{*}, H^{*}(y)$ sum of the square of the principal curvature of $\Gamma^{*}$, and $[g]:=g\left(h^{+}\left(v^{*}\right), v^{*}\right)-g\left(h^{-}\left(v^{*}\right), v^{*}\right)$.

Then, there is an $\epsilon_{0}>0$ such that (1.4) have an $\epsilon$-family of stationary solutions $\left(u^{\epsilon}, v^{\epsilon}\right)$ for $\epsilon \in\left(0, \epsilon_{0}\right]$ satisfying the following:

(i) $\lim _{\epsilon \rightarrow 0} v^{\epsilon}(x)=v^{*}$ uniformly on $\bar{\Omega}$.

(ii) For each $\delta>0$,

$$
\lim _{\epsilon \rightarrow 0} u^{\epsilon}(x)=\left\{\begin{array}{ll}
h^{-}\left(v^{*}\right), & x \in \bar{\Omega}_{-}\left(\Gamma^{*}\right) \backslash \Gamma_{\delta}^{*}, \\
h^{+}\left(v^{*}\right), & x \in \bar{\Omega}_{+}\left(\Gamma^{*}\right) \backslash \Gamma_{\delta}^{*},
\end{array} \quad\right. \text { uniformly }
$$

where $\Gamma_{\delta}^{*}$ is a tubular neighborhood of $\Gamma^{*}$.

(iii) For each $K>0$,

$$
\lim _{\epsilon \rightarrow 0} u^{\epsilon}(y+\epsilon \xi \nu(y))=u^{*}\left(\xi+s^{*}(y)\right) \quad \text { in } C^{2}[-K, K],
$$

uniformly in $y \in \Gamma^{*}$ for some $s^{*} \in C^{2, \alpha}\left(\Gamma^{*}\right)$.

The associated linearized problem of (1.4) is of the form

$$
\begin{cases}\lambda^{\epsilon} w=\epsilon^{2} \Delta w+f_{u}^{\epsilon} w-z, & \text { in } \Omega, \\ \epsilon \lambda^{\epsilon} z=D \Delta z+\epsilon g_{u}^{\epsilon} w+\epsilon g_{v}^{\epsilon} z, & \\ \frac{\partial w}{\partial n}=0=\frac{\partial z}{\partial n} & \text { on } \partial \Omega .\end{cases}
$$

Here we focus only on the critical eigenvalues. So we assume the following for (5.4):

$\left(\mathrm{A} 6^{\prime}\right)$ There exists an integer $m^{*} \geq 1$ such that each eigenvalue $\lambda^{\epsilon}$ and the associated eigenfunctions $\left(w^{\epsilon}, z^{\epsilon}\right)$ of (5.4) have the following asymptotic forms:

$$
\begin{cases}\lambda^{\epsilon}=\epsilon \lambda_{1}+\epsilon^{2} \lambda_{2}+o\left(\epsilon^{2}\right), & \\ w^{\epsilon}(x)=\sum_{j=0}^{m^{*}} \epsilon^{j} W^{ \pm, j}(x)+\omega(r) \cdot \sum_{j=0}^{m^{*}} \epsilon^{j} w^{ \pm, j}(r / \epsilon, y)+o\left(\epsilon^{m^{*}}\right), & \text { in } \Omega_{ \pm}\left(\Gamma^{*}\right), \\ z^{\epsilon}(x)=\sum_{j=0}^{m^{*}} \epsilon^{j} Z^{ \pm, j}(x)+\omega(r) \cdot \epsilon^{2} \sum_{j=0}^{m^{*}} \epsilon^{j} z^{ \pm, j}(r / \epsilon, y)+o\left(\epsilon^{m^{*}}\right), & \end{cases}
$$

where $W^{ \pm, i}(x) \in C^{2, \alpha}\left(\bar{\Omega}_{ \pm}\left(\Gamma^{*}\right)\right)$ and $Z^{ \pm, i}(x) \in C^{2, \alpha}\left(\bar{\Omega}_{ \pm}\left(\Gamma^{*}\right)\right)$ are outer expansions, $w^{ \pm, i}(\xi, y)$ and $z^{ \pm, j}(\xi, y)$ are inner expansions bounded for $\pm \xi \in$ $(0, \infty)$ and $y \in \Gamma^{*}$. 
In the activator-inhibitor case, the principal parts of $C^{1}$-matching conditions to eigenfunctions become

$$
\begin{aligned}
u_{\xi}^{*}\left(s_{0}(y)\right)\left[w_{\xi}^{-, 0}(0, y)-w_{\xi}^{+, 0}(0, y)\right] & =m^{2} \lambda_{1} \theta(y)-J^{\prime}\left(v^{*}\right) q_{0}(y)=0, \\
Z_{r}^{-, 0}(0, y)-Z_{r}^{+, 0}(0, y) & =\left(\Pi_{-}+\Pi_{+}\right) q_{0}=0 .
\end{aligned}
$$

Then, the following two cases are considered.

Case I. $q_{c} \neq 0$ and $\theta$ is a constant function $\theta(y)=\theta_{c}(\neq 0)$. Then $\lambda_{1}$ is given by

$$
\lambda_{1}=\frac{J^{\prime}\left(v^{*}\right) q_{c}}{m^{2} \theta_{c}} .
$$

Case II. $q_{c}=0$ and $\lambda_{1}=0$.

Concerning Case I, we have the following a priori estimate for $\lambda_{1}$.

Lemma 5.1 (a priori estimate for $\lambda_{1}$ ). Assume that the principal eigenvalue $\lambda^{\epsilon}$ and the associated eigenfunctions have the following asymptotic forms:

$$
\begin{gathered}
\lambda^{\epsilon}=\epsilon \lambda_{1}(\epsilon) \quad \text { and } \quad \lim _{\epsilon \downarrow 0} \lambda_{1}(\epsilon)=\frac{J^{\prime}\left(v^{*}\right) q_{c}}{m^{2} \theta_{c}}, \\
w \approx \epsilon h_{v}^{ \pm}\left(v^{*}\right) q_{c}+\left(w^{0}(r / \epsilon, y)+\epsilon w^{ \pm, 1}(r / \epsilon, y)\right) \cdot \omega(r), \\
z \approx q_{c}+\epsilon Z^{ \pm, 2}(x)+\epsilon^{2}\left(z^{ \pm, 1}(r / \epsilon, y)+\epsilon z^{ \pm, 2}(r / \epsilon, y)\right) \cdot \omega(r)
\end{gathered}
$$

on $\Omega_{ \pm}\left(\Gamma^{*}\right)$. Then the real part of $\lambda_{1}(\epsilon)$ is strictly negative.

Proof. See Appendix G.

In view of Lemma 5.1, we expand $\lambda^{\epsilon}$ as $\lambda^{\epsilon}=\epsilon^{2} \lambda_{2}+o\left(\epsilon^{2}\right)$. For the eigenvalues of (5.4), we have the following result.

Corollary of Theorem 1.3. Assume that (A1)-(A3) in section 1 , (A4')-(A6 $\left.{ }^{\prime}\right)$ with $\lambda_{1}=0$ and (A7) are satisfied. Then the principal part of the critical eigenvalues is given by $\epsilon^{2} \lambda^{*}$, where $\lambda^{*}$ is the eigenvalue of the following problem:

$$
L \theta^{*}=\lambda^{*} \theta^{*}
$$

for $\theta^{*} \in(I-P) C^{2, \alpha}\left(\Gamma^{*}\right)$.

We assume, for simplicity, that the nonlinearity takes the form $(f, g)=\left(u-u^{3}\right.$, $u-\gamma v), \gamma>3 / 2$. Then the constants are precisely computed as

$$
v^{*}=0, \quad h^{ \pm}\left(v^{*}\right)= \pm 1, \quad J^{\prime}\left(v^{*}\right)=-2, \quad m^{2}=\frac{2 \sqrt{2}}{3}=\frac{4}{3 \sqrt{2}} .
$$

For example, when $\Omega$ is a rectangle in $(x, y)$-plane $\Omega=(0, X) \times(0, Y)$, the reduced ploblems (5.1)-(5.3) are recast as

$$
\begin{gathered}
\left\{\begin{array}{l}
D V_{x x}^{+}+G^{+}=0, \quad x_{0}<x<X, \\
V^{+}\left(x_{0}\right)=0, \quad V_{x}^{+}(X)=0,
\end{array}\right. \\
\left\{\begin{array}{l}
D V_{x x}^{-}+G^{-}=0, \quad 0<x<x_{0}, \\
V_{x}^{-}(0)=0, \quad V^{-}\left(x_{0}\right)=0
\end{array}\right.
\end{gathered}
$$




$$
V_{x}^{-}\left(x_{0}\right)=V_{x}^{+}\left(x_{0}\right)
$$

where $G^{ \pm}:=g\left(h^{ \pm}\left(v^{*}\right), v^{*}\right)= \pm 1, \Omega_{+}\left(\Gamma^{*}\right)=\left\{(x, y) \in \mathbf{R}^{2} \mid x_{0}<x<X, 0<y<Y\right\}$, $\Omega_{-}\left(\Gamma^{*}\right)=\left\{(x, y) \in \mathbf{R}^{2} \mid 0<x<x_{0}, 0<y<Y\right\}$, and $\Gamma^{*}=\left\{\left(x_{0}, y\right) \in \mathbf{R}^{2} \mid 0<y<\right.$ $Y\}$. Here we used the fact that $H^{*}(y)=0$. We can easily show that $(5.6)_{-}$and $(5.6)_{+}$ have unique solutions given by

$$
\begin{aligned}
V^{-}(x) & =-\frac{1}{2 D} G^{-}\left[x^{2}-x_{0}^{2}\right], \\
V^{+}(x) & =-\frac{G^{+}}{D}\left[\frac{1}{2}\left(x^{2}-x_{0}^{2}\right)-X\left(x-x_{0}\right)\right] .
\end{aligned}
$$

Then, by using the $C^{1}$-matching condition (5.7), we can uniquely determine $x_{0}$ and $V_{x}\left(x_{0}\right)$ as

$$
x_{0}=\frac{G^{+} X}{[g]} \text { and } V_{x}\left(x_{0}\right)=-\frac{G^{+} G^{-} X}{D[g]},
$$

where $[g]=G^{+}-G^{-}$. The existence results of the planar solution to (1.4) is given by Taniguchi and Nishiura [23] (see Theorem 4.6 in section 4).

The $j$ th eigenvalue $\Lambda_{j}$ of $\mathcal{T}$ is given by $\Lambda_{j}=X \Lambda(\kappa \pi j)$, where

$$
\Lambda(z)=\frac{\cosh z+\cosh p z}{2 z \sinh z}, p=-\frac{G^{-}}{[g]}, \quad \kappa=\frac{X}{Y} .
$$

Then the associated eigenfunction is $\beta_{j}(y)=\cos \left(\tau_{j} y\right)$, where $\tau_{j}=\pi j / Y$. Therefore, the $j$ th eigenvalue of $L$ is real and given by $\Sigma_{j}=\Sigma(\kappa \pi j, X, D)$, where

$$
\Sigma(z, X, D):=-\left(\frac{z}{X}\right)^{2}-\frac{J^{\prime}\left(v^{*}\right) X}{D m^{2}}\left[-\frac{G^{+} G^{-}}{[g]}+[g] \Lambda(z)\right] .
$$

The associated eigenfunction is the same as that of $\mathcal{T} . \Sigma(z, X, D)$ have the following properties.

Lemma 5.2. The nullcline of $\Sigma(z, X, D)$ as a function of $z>0$ and $X>0$ is given by $\left\{(z, X(z, D)) \mid z>z_{0}\right\}$, where $z_{0}$ is a unique zero of $-\frac{G^{+} G^{-}}{[g]}+[g] \Lambda(z)=0$ and

$$
X(z, D)=\left(\frac{D m^{2}}{-J^{\prime}\left(v^{*}\right)}\right)^{1 / 3}\left[-\frac{G^{+} G^{-}}{[g]}+[g] \Lambda(z)\right]^{-1 / 3} z^{2 / 3} .
$$

Moreover, $X(z, D)$ has the following properties:

$$
\begin{gathered}
\lim _{z \rightarrow z_{0}+0} X(z, D)=\infty, \\
\lim _{z \rightarrow \infty}\left[X(z, D)-\left(\frac{D m^{2}}{-J^{\prime}\left(v^{*}\right)}\right)^{1 / 3}\left(-\frac{G^{+} G^{-}}{[g]}\right)^{-1 / 3} z^{2 / 3}\right]=0, \\
\frac{d X}{d z}\left\{\begin{array}{lll}
<0 & \text { for } & z_{0}<z<z_{1} \\
=0 & \text { for } & z=z_{1} \\
>0 & \text { for } & z_{1}<z,
\end{array}\right.
\end{gathered}
$$

for some $z_{1}=z_{1}(D)$. 
By using Lemma 5.2, we obtain the following corollary.

Corollary of TheOrem 1.5. (i) For any $D>0$, there exists $\underline{X}=\underline{X}(D)>0$ such that the planar solution is stable for $X<\underline{X}$ and $\kappa>0$.

(ii) For any fixed $\kappa>0$ and $D>0$, there exists $\bar{X}=\bar{X}(\kappa, D)>0$ such that the planar solution is unstable for $X>\bar{X}$.

Note. After finishing our paper, the anonymous referee noted us an interesting preprint, "On the spectra of 3-D lamellar solutions of the diblock copolymer problem," by X. Ren and J. Wei, which now appears in [27]. Their results are consistent with the part of our stability results for the lamellar patterns, although they employed a different Euler-Lagrange equation and methods for stability analysis.

Appendix A. (proof of Proposition 2.2). First we compute $u_{\xi}^{ \pm, 2}(0, y)$.

$$
\begin{aligned}
u_{\xi}^{ \pm, 2}(0, y)= & -U^{ \pm, 2}(0, y) \frac{u_{\xi \xi}^{*}\left(s_{0}\right)}{u_{\xi}^{*}\left(s_{0}\right)} \\
& -\frac{1}{u_{\xi}^{*}\left(s_{0}\right)} \int_{ \pm \infty}^{0}\left[p_{2,1}^{ \pm}+p_{2,2}^{ \pm}+p_{2,3}^{ \pm}+p_{2,4}^{ \pm}+p_{2,5}^{ \pm}\right] u_{\xi}^{*}\left(\xi+s_{0}\right) d \xi
\end{aligned}
$$

where

$$
\begin{aligned}
& p_{2,1}^{ \pm}=(N-1) H(0, y) u_{\xi}^{1}, \\
& p_{2,2}^{ \pm}=\Delta(0) u^{0}+(N-1) H_{r}(0, y) \xi u_{\xi}^{0}, \\
& p_{2,3}^{ \pm}=\frac{1}{2} \tilde{f}_{u u}\left[U^{1}(0, y)+u^{1}\right]^{2}, \\
& p_{2,4}^{ \pm}=\tilde{f}_{u}\left[\xi U_{r}^{1}(0, y)+U^{2}(0, y)\right], \\
& p_{2,5}^{ \pm}=-\left[\xi V_{r}^{1}(0, y)+V^{2}(0, y)\right] .
\end{aligned}
$$

In the following, we compute each term of the integral part in (A.1):

$$
\begin{aligned}
& \int_{ \pm \infty}^{0} p_{2,2}(\xi, y) u_{\xi}^{*}\left(\xi+s_{0}\right) d \xi=\Delta(0) s_{0} \int_{ \pm \infty}^{s_{0}}\left[u_{\xi}^{*}(\xi)\right]^{2} d \xi+(N-1) H_{r}(0, y) \\
& \int_{ \pm \infty}^{s_{0}}\left(\xi-s_{0}\right)\left[u_{\xi}^{*}(\xi)\right]^{2} d \xi \int_{ \pm \infty}^{0} p_{2,3}(\xi, y) u_{\xi}^{*}\left(\xi+s_{0}\right) d \xi \\
& \quad=-\frac{1}{2} f_{u}^{*}\left[U^{1}(0, y)\right]^{2}-\int_{ \pm \infty}^{0} f_{u}\left(u^{*}\left(\xi+s_{0}\right)\right)\left[U^{1}(0, y)+u^{1}(\xi, y)\right] u_{\xi}^{1}(\xi, y) d \xi \\
& \quad=-\frac{1}{2} f_{u}^{*}\left[U^{1}(0, y)\right]^{2}+\int_{ \pm \infty}^{0}\left[u_{\xi \xi}^{1}(\xi, y)+(N-1) H(0, y) u_{\xi}^{*}\left(\xi+s_{0}\right)\right] u_{\xi}^{1}(\xi, y) d \xi \\
& \quad=\frac{1}{2} U^{1}(0, y) b_{1}^{*}(y)+\frac{1}{2}\left[u_{\xi}^{1, \pm}(0, y)\right]^{2}+(N-1) H(0, y) \int_{ \pm \infty}^{0} u_{\xi}^{1}(\xi, y) u_{\xi}^{*}\left(\xi+s_{0}\right) d \xi
\end{aligned}
$$

where $f_{u}^{*}=\frac{d}{d u} f\left(h^{ \pm}\left(v^{*}\right)\right)$ and we used the fact that $f_{u}^{*} U^{1}(y, 0)-b_{1}^{*}(y)=0$.

$$
\begin{aligned}
\int_{ \pm \infty}^{0} p_{2,4}(\xi, y) u_{\xi}^{*}\left(\xi+s_{0}\right) d \xi & =f\left(u^{*}\left(s_{0}\right)\right) U^{2}(0, y)-U_{r}^{1}(0, y) \int_{ \pm \infty}^{0} f\left(u^{*}\left(\xi+s_{0}\right)\right) d \xi \\
& =f\left(u^{*}\left(s_{0}\right)\right) U^{2}(0, y)+U_{r}^{1}(0, y) u_{\xi}^{*}\left(s_{0}\right) \\
\int_{ \pm \infty}^{0} p_{2,5}(\xi, y) u_{\xi}^{*}\left(\xi+s_{0}\right) d \xi & =-V_{r}^{1}(0, y) \int_{ \pm \infty}^{s_{0}}\left(\xi-s_{0}\right) u_{\xi}^{*}(\xi) d \xi-b_{2}(y)\left[u^{*}\left(s_{0}\right)-h^{ \pm}\left(v^{*}\right)\right]
\end{aligned}
$$


Concerning $p_{2,1}$, we have

$$
\begin{aligned}
\int_{ \pm \infty}^{0} u_{\xi}^{1}(\xi, y) u_{\xi}^{*}\left(\xi+s_{0}\right) d \xi=-\frac{U^{1}(0, y)}{u_{\xi}^{*}\left(s_{0}\right)} \int_{ \pm \infty}^{0} u_{\xi \xi}^{*}\left(\xi+s_{0}\right) u_{\xi}^{*}\left(\xi+s_{0}\right) d \xi \\
-\int_{ \pm \infty}^{0} u_{\xi \xi}^{*}\left(\xi+s_{0}\right) u_{\xi}^{*}\left(\xi+s_{0}\right) d \xi \int_{0}^{\xi}\left[u_{\xi}^{*}\left(\tau+s_{0}\right)\right]^{-2} \int_{ \pm \infty}^{\tau}[* * *] u_{\xi}^{*}\left(s+s_{0}\right) d s d \tau d \xi \\
-\int_{ \pm \infty}^{0} \int_{ \pm \infty}^{\xi}[* * *] u_{\xi}^{*}\left(s+s_{0}\right) d \tau d \xi \\
=\frac{1}{2} U^{1, \pm}(0, y) u_{\xi}^{*}\left(s_{0}\right)-\frac{1}{2} \int_{ \pm \infty}^{0} \int_{ \pm \infty}^{\xi}\left[(N-1) H(0, y) u_{\xi}^{*}\left(\tau+s_{0}\right)\right. \\
\quad+\frac{f_{u}\left(u^{*}\left(\tau+s_{0}\right)\right) U^{1}(0, y)}{-}\left(b_{1}^{*}(y)\right] u_{\xi}^{*}\left(\tau+s_{0}\right) d \tau d \xi \\
=-\frac{1}{2} \int_{ \pm \infty}^{0} 1 \int_{ \pm \infty}^{\xi}\left[(N-1) H(0, y) u_{\xi}^{*}\left(\tau+s_{0}\right)-b_{1}^{*}(y)\right] u_{\xi}^{*}\left(\tau+s_{0}\right) d \tau d \xi \\
=\frac{1}{2} \int_{ \pm \infty}^{s_{0}}\left(\xi-s_{0}\right)\left[(N-1) H(0, y) u_{\xi}^{*}(\xi)-b_{1}^{*}(y)\right] u_{\xi}^{*}(\xi) d \xi
\end{aligned}
$$

The underlined terms cancel pairwise. By using the above expression,

$$
\begin{gathered}
\int_{-\infty}^{0} u_{\xi}^{-, 1}(\xi, y) u_{\xi}^{*}\left(\xi+s_{0}\right) d \xi-\int_{\infty}^{0} u_{\xi}^{+, 1}(\xi, y) u_{\xi}^{*}\left(\xi+s_{0}\right) d \xi \\
=\frac{1}{2} \int_{-\infty}^{\infty} \xi\left[(N-1) H(0, y) u_{\xi}^{*}(\xi)-b_{1}^{*}(y)\right] u_{\xi}^{*}(\xi) d \xi
\end{gathered}
$$

Here we used the fact that $b_{1}^{*}(y)=-m^{2}(N-1) H(0, y) / J^{\prime}\left(v^{*}\right)$.

Combining the above computations, we have

$$
\begin{aligned}
& -u_{\xi}^{*}\left(s_{0}\right)\left[u_{\xi}^{ \pm, 2}(0, y)+U_{r}^{ \pm, 1}(0, y)\right] \\
& =2(N-1) H(0, y) \int_{ \pm \infty}^{0} u_{\xi}^{ \pm, 1}(\xi, y) u_{\xi}^{*}\left(\xi+s_{0}\right) d \xi \\
& \quad+\left[\Delta(0) s_{0}-(N-1) H_{r}(0, y) s_{0}\right] \int_{ \pm \infty}^{s_{0}}\left[u_{\xi}^{*}(\xi)\right]^{2} d \xi+(N-1) H_{r}(0, y) \int_{ \pm \infty}^{s_{0}} \xi\left[u_{\xi}^{*}(\xi)\right]^{2} d \xi \\
& \quad+\frac{1}{2} U^{ \pm, 1}(0, y) b_{1}^{*}(y)+\frac{1}{2}\left[u_{\xi}^{ \pm, 1}(0, y)\right]^{2}+s_{0} V_{r}^{1}(0, y) \int_{ \pm \infty}^{s_{0}} u_{\xi}^{*}(\xi) d \xi \\
& \quad-V_{r}^{1}(0, y) \int_{ \pm \infty}^{s_{0}} \xi u_{\xi}^{*}(\xi) d \xi-b_{2}(y) \int_{ \pm \infty}^{s_{0}} u_{\xi}^{*}(\xi) d \xi \\
& u_{\xi}^{*}\left(s_{0}\right)\left[u_{\xi}^{+, 2}(0, y)+U_{r}^{+, 1}(0, y)-u_{\xi}^{-, 2}(0, y)-U_{r}^{-, 1}(0, y)\right] \\
& =(N-1) \int_{-\infty}^{\infty} \xi\left[(N-1) H(0, y) u_{\xi}^{*}(\xi)-b_{1}^{*}(y)\right] u_{\xi}^{*}(\xi) d \xi \\
& +\left[\Delta(0) s_{0}-(N-1) H_{r}(0, y) s_{0}\right] \int_{-\infty}^{\infty}\left[u_{\xi}^{*}(\xi)\right]^{2} d \xi+(N-1) H_{r}(0, y) \int_{-\infty}^{\infty} \xi\left[u_{\xi}^{*}(\xi)\right]^{2} d \xi \\
& -\frac{1}{2} b_{1}^{*}(y)\left[U^{+, 1}(0, y)-U^{-, 1}(0, y)\right]+s_{0} V_{r}^{1}(0, y)[h]-V_{r}^{1}(0, y) \int_{-\infty}^{\infty} \xi u_{\xi}^{*}(\xi) d \xi-b_{2}(y)[h] .
\end{aligned}
$$

Substituting the above results into $\frac{\partial}{\partial r} \mathcal{U}^{+, \epsilon}(0, y)-\frac{\partial}{\partial r} \mathcal{U}^{-, \epsilon}(0, y)$, we have (2.9). 
Concerning (2.8), we note only the following:

$$
\begin{aligned}
v_{\xi}^{ \pm, 3}(\xi, y) & =-\int_{ \pm \infty}^{s_{0}}\left[u^{*}(\tau)-h^{ \pm}\left(v^{*}\right)\right] d \tau \\
& =-\int_{ \pm \infty}^{0}\left[u^{*}(\tau)-h^{ \pm}\left(v^{*}\right)\right] d \tau-\int_{0}^{s_{0}} u^{*}(\tau) d \tau+h^{ \pm}\left(v^{*}\right) s_{0}, \\
v_{\xi}^{-, 3}(\xi, y)-v_{\xi}^{+, 3}(\xi, y) & =-\int_{-\infty}^{0}\left[u^{*}(\tau)-h^{-}\left(v^{*}\right)\right] d \tau+\int_{\infty}^{0}\left[u^{*}(\tau)-h^{+}\left(v^{*}\right)\right] d \tau-[h] s_{0} .
\end{aligned}
$$

Appendix B. (proof of Lemma 3.1). We can assume without loss of generality that the $O(1)$-terms $W^{0}, Z^{0}, w^{0}$ and $z^{0}$ are not equivalent to zero at the same time. We note that when $\lambda^{\epsilon}=\lambda_{0}+\epsilon \lambda_{1}$, the expansions for $w$ and $C^{1}$-matching conditions are the same as in subsections $3.1,3.2$, and 3.3. Also, we easily see that $z^{0} \equiv 0$ and $z^{1} \equiv 0$ (see (3.9) and (3.10)) and the equation for $z^{ \pm, 2}$ becomes

$$
\left\{\begin{array}{l}
z_{\xi \xi}^{ \pm, 2}+\lambda_{0} w^{ \pm, 0}=0, \\
z^{ \pm, 2}(0, y)=0, \quad \lim _{\xi \rightarrow \pm \infty} z^{ \pm, 2}(\xi, y)=0 .
\end{array}\right.
$$

If $\lambda^{\epsilon}=\lambda_{0}+\epsilon \lambda_{1}$, the equations of $W^{0}$ and $Z^{0}$ become

$$
\left\{\begin{array}{l}
f_{u}^{0} W^{0}-Z^{0}=0, \\
\Delta Z^{0}+\lambda_{0} W^{0}=0,
\end{array} \quad \text { in } \Omega_{ \pm}\left(\Gamma^{*}\right),\right.
$$

and these equations are rewritten as

$$
\Delta Z^{0}+\frac{1}{f_{u}^{0}} \lambda_{0} Z^{0}=0
$$

since $f_{u}^{0}<0$. On the other hand, we see $Z^{0}(0, y)=q_{0}(y) \equiv 0$ from Lemma 3.4. So we conclude (i) $Z^{0} \equiv 0$ (hence $W^{0} \equiv 0$ ) and $\operatorname{Re} \lambda_{0} \geq 0$, or (ii) $Z^{0} \neq 0$ and $\operatorname{Re} \lambda_{0}<0$. If $Z^{0} \equiv 0$, the equation of $Z^{1}$ becomes

$$
\Delta Z^{1}+\frac{1}{f_{u}^{0}} \lambda_{0} Z^{1}=0
$$

Then the $O(\epsilon)$-term of $C^{1}$-matching conditions (3.19) become

$$
\begin{gathered}
u_{\xi}^{*}\left(s_{0}(y)\right)\left[W_{r}^{-, 0}(0, y)+w_{\xi}^{-, 1}(0, y)-W_{r}^{+, 0}(0, y)-w_{\xi}^{+, 1}(0, y)\right]=q_{1}(y)[h]=0, \\
Z_{r}^{-, 1}(0, y)+z_{\xi}^{-, 2}(0, y)-Z_{r}^{+, 1}(0, y)-z_{\xi}^{+, 2}(0, y) \\
=\left(\Pi_{-}+\Pi_{+}\right) q_{1}-\lambda_{0}[h] \theta(y)=0 .
\end{gathered}
$$

From (B.1) and (B.2), we have $Z^{1}(0, y)=q_{1}(y) \equiv 0$, and (a) $\lambda_{0}=0$ or (b) $\lambda_{0} \neq 0$ and $\theta(y) \equiv 0$. In the case (b), we obtain that $w^{0} \equiv 0$ since $w^{0}$ is given by

$$
w^{0}(\xi, y)=\Theta(y) \frac{u_{\xi}^{*}\left(\xi+s_{0}\right)}{u_{\xi}^{*}\left(s_{0}\right)}=\theta(y) u_{\xi}^{*}\left(\xi+s_{0}\right)
$$


(see (3.14)). This contradicts our assumption. Thus we conclude that $\operatorname{Re} \lambda_{0}<0$ or $\lambda_{0}=0$.

Appendix C. (proof of Lemma 3.5). $\tilde{\Phi}\left(\theta, q_{2}, \lambda_{1}, 0\right)=0$ is equivalent to

$$
u_{\xi}^{*}\left(s_{0}(y)\right)\left[w_{\xi}^{-, 2}(0, y)-w_{\xi}^{+, 2}(0, y)\right]=0 .
$$

In order to calculate $w_{\xi}^{ \pm, 2}(0, y)$, we display the equation and the boundary conditions of $w_{\xi}^{ \pm, 2}$ again

$$
\begin{gathered}
w_{\xi \xi}^{2}+(N-1) H(0, y) w_{\xi}^{1}+\left[(N-1) H_{r}(0, y) \xi \partial_{\xi}+\Delta^{\Gamma}\right] w^{0} \\
+\tilde{f}_{u}^{0}\left[w^{2}+W^{2}(0, y)\right]+\tilde{f}_{u}^{1} w^{1}+\tilde{f}_{u}^{2} w^{0}-Z^{2}(0, y)=0 \\
w^{ \pm, 2}(0, y)=-W^{ \pm, 2}(0, y), \quad \lim _{\xi \rightarrow \pm \infty} w^{ \pm, 2}(\xi, y)=0
\end{gathered}
$$

where

$$
\begin{gathered}
\tilde{f}_{u}^{ \pm, 2}=\tilde{f}_{u u}\left[p_{2,1}\right]+\frac{1}{2} \tilde{f}_{u u u}\left[p_{2,3}\right]^{2} \\
p_{2,1}:=\xi U_{r}^{1}(0, y)+U^{2}(0, y)+u^{2}, \quad p_{2,3}:=U^{1}(0, y)+u^{1} .
\end{gathered}
$$

Using the expression (3.17) for $w^{ \pm, 2}(\xi, y)$, we have

$$
\begin{aligned}
w_{\xi}^{2}(0, y)= & -\frac{1}{u_{\xi}^{*}\left(s_{0}\right)} \int_{ \pm \infty}^{0}\left[(N-1) H(0, y) \underline{w_{\xi}^{1}(\mathrm{i})}+(N-1) H_{r}(0, y) \underline{\xi \partial_{\xi} w^{0}}\right. \text { (ii) } \\
& +\underline{\Delta^{\Gamma^{*}} w^{0}} \text { (iii) }+\underline{\tilde{f}_{u}^{1} w^{1}(\mathrm{iv})}+\underline{\left.\tilde{f}_{u}^{2} w_{(\mathrm{v})}^{0}-Z^{2}(0, y)\right] u_{\xi}^{*}\left(s+s_{0}\right) d \xi}
\end{aligned}
$$

In the following, we calculate each term of integral in (C.1):

Computation of (i). By using

$$
\begin{aligned}
w^{ \pm, 1}(\xi, y)= & -\theta(y) u_{\xi}^{*}\left(\xi+s_{0}\right) \int_{0}^{\xi}\left[u_{\xi}^{*}\left(t+s_{0}\right)\right]^{-2} \\
& \times \int_{ \pm \infty}^{t}\left[(N-1) H(0, y) u_{\xi \xi}^{*}\left(s+s_{0}\right)+\tilde{f}_{u}^{1} u_{\xi}^{*}\left(s+s_{0}\right)\right] u_{\xi}^{*}\left(s+s_{0}\right) d s d t
\end{aligned}
$$

we have

$$
\begin{aligned}
\int_{ \pm \infty}^{0} w_{\xi}^{1}(\xi, y) u_{\xi}^{*}\left(\xi+s_{0}\right) d \xi= & -\int_{ \pm \infty}^{0} w^{1}(\xi, y) u_{\xi \xi}^{*}\left(\xi+s_{0}\right) d \xi \\
= & -\frac{1}{2} \theta(y) \int_{ \pm \infty}^{0} \int_{ \pm \infty}^{\xi}\left[(N-1) H(0, y) u_{\xi \xi}^{*}\left(s+s_{0}\right)\right. \\
& \left.+\tilde{f}_{u}^{1} u_{\xi}^{*}\left(s+s_{0}\right)\right] u_{\xi}^{*}\left(s+s_{0}\right) d s d \xi \\
= & \frac{1}{2} \theta(y)\left[u_{\xi}^{ \pm, 1}(0, y) u_{\xi}^{*}\left(s_{0}\right)-2 I_{3}^{ \pm}\right] .
\end{aligned}
$$

Here we used the fact that

$$
\begin{aligned}
& -\int_{ \pm \infty}^{0} \int_{ \pm \infty}^{\xi}\left[(N-1) H(0, y) u_{\xi \xi}^{*}\left(s+s_{0}\right)+\tilde{f}_{u}^{1} u_{\xi}^{*}\left(s+s_{0}\right)\right] u_{\xi}^{*}\left(s+s_{0}\right) d s d \xi \\
& =u_{\xi}^{ \pm, 1}(0, y) u_{\xi}^{*}\left(s_{0}\right)-2 I_{3}^{ \pm}
\end{aligned}
$$


where

$$
I_{3}^{ \pm}:=\int_{ \pm \infty}^{0} u_{\xi}^{1}(\xi, y) u_{\xi \xi}^{*}\left(\xi+s_{0}\right) d \xi
$$

Equation (C.2) is proved in Appendix D.

Computation of (ii).

$$
\begin{aligned}
\int_{ \pm \infty}^{0} \xi w_{\xi}^{0}(\xi, y) u_{\xi}^{*}\left(\xi+s_{0}\right) d \xi & =\theta(y) \int_{ \pm \infty}^{0} \xi u_{\xi \xi}^{*}\left(\xi+s_{0}\right) u_{\xi}^{*}\left(\xi+s_{0}\right) d \xi \\
& =-\frac{1}{2} \theta(y) \int_{ \pm \infty}^{0}\left[u_{\xi}^{*}\left(\xi+s_{0}\right)\right]^{2} d \xi
\end{aligned}
$$

Computation of (iii).

$$
\begin{aligned}
\Delta^{\Gamma^{*}} w^{0} & =\Delta^{\Gamma^{*}}\left(\theta u_{\xi}^{*}\right) \\
& =u_{\xi}^{*} \Delta^{\Gamma^{*}} \theta+2 u_{\xi \xi}^{*} \nabla^{\Gamma} \theta \cdot \nabla s_{0}+u_{\xi \xi}^{*} \theta \Delta^{\Gamma^{*}} s_{0}+u_{\xi \xi \xi}^{*} \theta\left|\nabla^{\Gamma} s_{0}\right|^{2} .
\end{aligned}
$$

Here note that $u_{\xi}^{*}, u_{\xi \xi}^{*}$, etc., are all evaluated at $\xi+s_{0}$. Then multiplying $\Delta^{\Gamma^{*}} w^{0}$ by $u_{\xi}^{*}$, we have

$$
\begin{aligned}
\int_{ \pm \infty}^{0}\left(\Delta^{\Gamma^{*}} w^{0}\right) u_{\xi}^{*} d \xi= & \Delta^{\Gamma^{*}} \theta \int_{ \pm \infty}^{0}\left[u_{\xi}^{*}\left(\xi+s_{0}\right)\right]^{2} d \xi+\left[u_{\xi}^{*}\left(s_{0}\right)\right]^{2}\left[\nabla^{\Gamma} \theta \cdot \nabla s_{0}+\frac{1}{2} \theta \Delta^{\Gamma^{*}} s_{0}\right] \\
& +\left[u_{\xi \xi}^{*}\left(s_{0}\right) u_{\xi}^{*}\left(s_{0}\right)-\int_{ \pm \infty}^{0}\left[u_{\xi \xi}^{*}\left(\xi+s_{0}\right)\right]^{2} d \xi\right] \theta\left|\nabla^{\Gamma} s_{0}\right|^{2} .
\end{aligned}
$$

Computation of (iv).

$$
\begin{aligned}
\int_{ \pm \infty}^{0} & \tilde{f}_{u}^{1} u_{\xi}^{*}\left(\xi+s_{0}\right) w^{1}(\xi, y) d s d \xi \\
= & -\int_{ \pm \infty}^{0}\left[(N-1) H(0, y) u_{\xi \xi}^{*}\left(\xi+s_{0}\right)+u_{\xi \xi \xi}^{1}(\xi, y)+\tilde{f}_{u} u_{\xi}^{1}(\xi, y)\right] w^{1}(\xi, y) d \xi \\
& \times\left(u_{\xi \xi \xi}^{1}+\tilde{f}_{u} u_{\xi}^{1}+(N-1) H(0, y) u_{\xi \xi}^{*}+\tilde{f}_{u}^{1} u_{\xi}^{*}=0 \text { is used }\right) \\
= & (N-1) H(0, y) \int_{ \pm \infty}^{0} u_{\xi}^{*}\left(\xi+s_{0}\right) w_{\xi}^{1}(\xi, y) d \xi+u_{\xi}^{ \pm, 1}(0, y) w_{\xi}^{ \pm, 1}(0, y) \\
& -\int_{ \pm \infty}^{0}\left[w_{\xi \xi}^{ \pm, 1}(\xi, y)+\tilde{f}_{u} w^{ \pm, 1}(\xi, y)\right] u_{\xi}^{ \pm, 1} d \xi \\
& \times\left(w_{\xi \xi}^{1}+\tilde{f}_{u} w^{1}+(N-1) H(0, y) w_{\xi}^{0}+\tilde{f}_{u}^{1} w^{0}=0 \text { and } w^{0}=\theta u_{\xi}^{*} \quad \text { will be used }\right) \\
= & (N-1) H(0, y) \int_{ \pm \infty}^{0} u_{\xi}^{*}\left(\xi+s_{0}\right) w_{\xi}^{1}(\xi, y) d \xi+u_{\xi}^{ \pm, 1}(0, y) w_{\xi}^{ \pm, 1}(0, y) \\
& +\theta(y) \int_{ \pm \infty}^{0}\left[(N-1) H(0, y) u_{\xi \xi}^{*}\left(\xi+s_{0}\right)+\tilde{f}_{u}^{1} u_{\xi}^{*}\left(\xi+s_{0}\right)\right] u_{\xi}^{ \pm, 1}(\xi, y) d \xi \\
= & (N-1) H(0, y) \int_{ \pm \infty}^{0} u_{\xi}^{*}\left(\xi+s_{0}\right) w_{\xi}^{1}(\xi, y) d \xi+u_{\xi}^{ \pm, 1}(0, y) w_{\xi}^{ \pm, 1}(0, y) \\
& +\theta(y)\left[(N-1) H(0, y) I_{3}^{ \pm}+I_{4}^{ \pm}\right]
\end{aligned}
$$


where

$$
I_{4}^{ \pm}:=\int_{ \pm \infty}^{0} \tilde{f}_{u}^{1} u_{\xi}^{*}\left(\xi+s_{0}\right) u_{\xi}^{ \pm, 1}(\xi, y) d \xi=\int_{ \pm \infty}^{0} \tilde{f}_{u u}\left[U^{1}(0, y)+u^{1}\right] u_{\xi}^{1}(\xi, y) u_{\xi}^{*}\left(\xi+s_{0}\right) d \xi
$$

Computation of (v).

$$
\begin{aligned}
& \int_{ \pm \infty}^{0} \tilde{f}_{u}^{ \pm, 2} w^{0} u_{\xi}^{*} d \xi=\theta(y) \int_{ \pm \infty}^{0} \tilde{f}_{u}^{ \pm, 2}\left[u_{\xi}^{*}\right]^{2} d \xi \\
& \int_{ \pm \infty}^{0} \tilde{f}_{u}^{ \pm, 2}\left[u_{\xi}^{*}\right]^{2} d \xi \\
& =\left[\tilde{f}_{u} u_{\xi}^{*} p_{2,1}\right]_{ \pm \infty}^{0}-\int_{ \pm \infty}^{0}\left[U_{r}^{1}(0, y)+u_{\xi}^{2}\right] \tilde{f}_{u} u_{\xi}^{*} d \xi-\underline{\int_{ \pm \infty}^{0} \tilde{f}_{u} p_{2,1} u_{\xi \xi}^{*} d \xi} A \\
& +\left[\frac{1}{2} \tilde{f}_{u u} u_{\xi}^{*}\left[p_{2,3}\right]^{2}\right]_{ \pm \infty}^{0} \underline{-\int_{ \pm \infty}^{0} \tilde{f}_{u u}\left[U^{1}(0, y)+u^{1}\right] u_{\xi}^{1} u_{\xi}^{*} d \xi} \underset{-{\underline{\int_{ \pm \infty}}}^{0} \frac{1}{2} \tilde{f}_{u u}\left[p_{2,3}\right]^{2} u_{\xi \xi}^{*} d \xi}{A} \\
& =-\int_{ \pm \infty}^{0}\left[u_{\xi \xi}^{ \pm, 2}+\underline{\left.\tilde{f}_{u} p_{2,1}+\frac{1}{2} \tilde{f}_{u u}\left[p_{2,3}\right]^{2}\right] u_{\xi \xi}^{*} d \xi} A\right. \\
& +u_{\xi \xi}^{*}\left(s_{0}\right)\left[U_{r}^{ \pm, 1}(0, y)+u_{\xi}^{ \pm, 2}(0, y)\right]-\underline{\int_{ \pm \infty}^{0} \tilde{f}_{u u}\left[U^{1}(0, y)+u^{1}\right] u_{\xi}^{1} u_{\xi}^{*} d \xi} C \\
& =\int_{ \pm \infty}^{0}\left[(N-1) H(0, y) u_{\xi}^{1}+\Delta(0) u^{0}+(N-1) H_{r}(0, y) \xi u_{\xi}^{0}\right] u_{\xi \xi}^{*} d \xi+V_{r}^{1}(0, y) \int_{ \pm \infty}^{0} u_{\xi}^{*} d \xi \\
& +u_{\xi \xi}^{*}\left(s_{0}\right)\left[U_{r}^{ \pm, 1}(0, y)+u_{\xi}^{ \pm, 2}(0, y)\right]-I_{4}^{ \pm}
\end{aligned}
$$

Here we used the equation of $u^{ \pm, 1}$.

$$
\begin{aligned}
\int_{ \pm \infty}^{0} & {\left[(N-1) H(0, y) u_{\xi}^{1}+\Delta(0) u^{0}+(N-1) H_{r}(0, y) \xi u_{\xi}^{0}\right] u_{\xi \xi}^{*} d \xi } \\
= & (N-1) H(0, y) I_{3}^{ \pm}+\left|\nabla^{\Gamma^{*}} s_{0}\right|^{2} \int_{ \pm \infty}^{0}\left[u_{\xi \xi}^{*}\left(\xi+s_{0}\right)\right]^{2} d \xi+\frac{1}{2}\left[u_{\xi}^{*}\left(s_{0}\right)\right]^{2} \Delta^{\Gamma} s_{0} \\
& -\frac{1}{2}(N-1) H_{r}(0, y) \int_{ \pm \infty}^{0}\left[u_{\xi}^{*}\left(\xi+s_{0}\right)\right]^{2} d \xi
\end{aligned}
$$

Here we used the fact that $\Delta(0) u^{0}=\left|\nabla^{\Gamma^{*}} s_{0}\right|^{2} u_{\xi \xi}^{*}+\Delta^{\Gamma^{*}} u_{\xi}^{*}$. 
Combining the above computations, we obtain

$$
\begin{aligned}
& -u_{\xi}^{*}\left(s_{0}\right) w_{\xi}^{ \pm, 2}(0, y)=\int_{ \pm}^{0} P_{2}(\xi, y) u_{\xi}^{*}\left(\xi+s_{0}\right) d \xi \\
& =\frac{1}{2}(N-1) H(0, y)\left[u_{\xi}^{ \pm, 1}(0, y) u_{\xi}^{*}\left(s_{0}\right)-2 I_{3}^{ \pm}\right] \theta \\
& -\frac{1}{2}(N-1) H_{r}(0, y)\left[m^{ \pm}\right]^{2} \theta \\
& +\left[m^{ \pm}\right]^{2} \Delta^{\Gamma^{*}} \theta+\left[u_{\xi}^{*}\left(s_{0}\right)\right]^{2}\left[\nabla^{\Gamma^{*}} \theta \cdot \nabla^{\Gamma^{*}} s_{0}+\frac{1}{2} \theta \Delta^{\Gamma^{*}} s_{0}\right] \\
& +\left[u_{\xi \xi}^{*}\left(s_{0}\right) u_{\xi}^{*}\left(s_{0}\right)-\left[n^{ \pm}\right]^{2}\right]\left|\nabla^{\Gamma^{*}} s_{0}\right|^{2} \theta \\
& +\frac{1}{2}(N-1) H(0, y)\left[u_{\xi}^{ \pm, 1}(0, y) u_{\xi}^{*}\left(s_{0}\right)-2 I_{3}^{ \pm}\right] \theta+u_{\xi}^{ \pm, 1}(0, y) w_{\xi}^{ \pm, 1}(0, y) \\
& +\left[(N-1) H(0, y) I_{3}^{ \pm}+I_{4}^{ \pm}\right] \theta \\
& +\left[(N-1) H(0, y) I_{3}^{ \pm}+\left|\nabla^{\Gamma^{*}} s_{0}\right|^{2}\left[n^{ \pm}\right]^{2}+\frac{1}{2}\left[u_{\xi}^{*}\left(s_{0}\right)\right]^{2} \Delta^{\Gamma^{*}} s_{0}-\frac{1}{2}(N-1) H_{r}(0, y)\left[m^{ \pm}\right]^{2}\right. \\
& \left.+u_{\xi \xi}^{*}\left(s_{0}\right)\left[U_{r}^{ \pm, 1}(0, y)+u_{\xi}^{ \pm, 2}(0, y)\right]-I_{4}^{ \pm}+V_{r}^{1}(0, y) \int_{ \pm \infty}^{0} u_{\xi}^{*}\left(\xi+s_{0}\right) d \xi\right] \theta \\
& -Z^{2}(0, y) \int_{ \pm \infty}^{0} u_{\xi}^{*}\left(\xi+s_{0}\right) d \xi \\
& =\left[m^{ \pm}\right]^{2} \Delta^{\Gamma^{*}} \theta-(N-1) H_{r}(0, y)\left[m^{ \pm}\right]^{2} \theta+V_{r}^{1}(0, y) \theta \int_{ \pm \infty}^{0} u_{\xi}^{*}\left(\xi+s_{0}\right) d \xi \\
& -Z^{2}(0, y) \int_{ \pm \infty}^{0} u_{\xi}^{*}\left(\xi+s_{0}\right) d \xi+(N-1) H(0, y) u_{\xi}^{ \pm, 1}(0, y) u_{\xi}^{*}\left(s_{0}\right) \theta \\
& +\left[u_{\xi}^{*}\left(s_{0}\right)\right]^{2}\left[\nabla^{\Gamma^{*}} \theta \cdot \nabla^{\Gamma^{*}} s_{0}+\theta \Delta^{\Gamma^{*}} s_{0}\right]+u_{\xi \xi}^{*}\left(s_{0}\right) u_{\xi}^{*}\left(s_{0}\right)\left|\nabla^{\Gamma^{*}} s_{0}\right|^{2} \theta \\
& +u_{\xi}^{ \pm, 1}(0, y) w_{\xi}^{ \pm, 1}(0, y)+u_{\xi \xi}^{*}\left(s_{0}\right)\left[U_{r}^{ \pm, 1}(0, y)+u_{\xi}^{ \pm, 2}(0, y)\right]
\end{aligned}
$$

where

$$
m^{ \pm}:=\left[\int_{ \pm \infty}^{0}\left[u_{\xi}^{*}\left(\xi+s_{0}\right)\right]^{2} d \xi\right]^{1 / 2} \text { and } n^{ \pm}:=\left[\int_{ \pm \infty}^{0}\left[u_{\xi \xi}^{*}\left(\xi+s_{0}\right)\right]^{2} d \xi\right]^{1 / 2}
$$

Finally, by using the relations

$$
\begin{aligned}
& u_{\xi}^{+, 1}(0, y)=u_{\xi}^{-, 1}(0, y), \quad w_{\xi}^{+, 1}(0, y)=w_{\xi}^{-, 1}(0, y), \\
& U_{r}^{+, 1}(0, y)+u_{\xi}^{+, 1}(0, y)=U_{r}^{-, 1}(0, y)+u_{\xi}^{-, 1}(0, y),
\end{aligned}
$$


we obtain

$$
\begin{aligned}
-u_{\xi}^{*}\left(s_{0}\right)\left[w_{\xi}^{-, 2}(0, y)-w_{\xi}^{+, 2}(0, y)\right] d= & m^{2} \Delta^{\Gamma^{*}} \theta+m^{2} H^{*}(y) \theta \\
& -V_{r}^{1}(0, y) J^{\prime}\left(v^{*}\right) \theta+J^{\prime}\left(v^{*}\right) Z^{2}(0, y),
\end{aligned}
$$

where

$$
H^{*}(y):=-(N-1) H_{r}(0, y)=\sum_{j=1}^{N-1}\left[\kappa_{j}\right]^{2},
$$

$\kappa_{j}(j=1, \ldots, N-1)$, are the principal curvatures of $\Gamma^{*}$. Thus we obtain (3.26).

Next we prove (3.27). By using the fact that $W^{ \pm, 0}(x) \equiv 0 \equiv Z^{ \pm, 0}(x)$ and $W^{ \pm, 1}(x) \equiv 0 \equiv Z^{ \pm, 1}(x)$, we see that $Z^{ \pm, 2}$ satisfies the following equation:

$$
\left\{\begin{array}{l}
\Delta Z^{ \pm, 2}=0 \quad \text { in } \Omega_{ \pm}\left(\Gamma^{*}\right), \\
Z^{ \pm, 2}=q_{2} \quad \text { on } \Gamma^{*}, \quad \frac{\partial Z^{-, 2}}{\partial n}=0 \quad \text { on } \partial \Omega .
\end{array}\right.
$$

Then we have

$$
Z_{r}^{-, 2}(0, y)-Z_{r}^{+, 2}(0, y)=\left.\frac{\partial}{\partial \nu}\left(\mathcal{P}^{-} q_{2}\right)\right|_{\Gamma^{*}}+\left.\frac{\partial}{\partial \nu}\left(\mathcal{P}^{+} q_{2}\right)\right|_{\Gamma^{*}}=\left(\Pi_{-}+\Pi_{+}\right) q_{2} .
$$

In view of (3.15) and (3.22), we have

$$
z_{\xi}^{-, 3}(0, y)-z_{\xi}^{+, 3}(0, y)=\left(1+\lambda_{1}\right)[h] \theta(y) .
$$

Combining (C.3) and (C.4), we obtain

$$
Z_{r}^{-, 2}(0, y)-Z_{r}^{+, 2}(0, y)+z_{\xi}^{-, 3}(0, y)-z_{\xi}^{+, 3}(0, y)=\left(\Pi_{-}+\Pi_{+}\right) q_{2}-\left(1+\lambda_{1}\right)[h] \theta(y) .
$$

Appendix D. (proof of (3.25) and (C.2)). By using

$$
u_{\xi \xi \xi}^{1}+\tilde{f}_{u} u_{\xi}^{1}+(N-1) H(0, y) u_{\xi \xi}^{*}+\tilde{f}_{u}^{1} u_{\xi}^{*}=0 \text { and } u_{\xi \xi \xi}^{*}+\tilde{f}_{u} u_{\xi}^{*}=0,
$$

we have

$$
\begin{aligned}
& \int_{ \pm \infty}^{0}\left[(N-1) H(0, y) u_{\xi \xi}^{*}\left(\xi+s_{0}\right)+\tilde{f}_{u}^{1} u_{\xi}^{*}\left(\xi+s_{0}\right)\right] u_{\xi}^{*}\left(\xi+s_{0}\right) d \xi \\
& \quad=u_{\xi}^{ \pm, 1}(0, y) u_{\xi \xi}^{*}\left(s_{0}\right)-u_{\xi}^{*}\left(s_{0}\right) u_{\xi \xi}^{ \pm, 1}(0, y)
\end{aligned}
$$

and

$$
\begin{aligned}
& -\int_{ \pm \infty}^{0} \int_{ \pm \infty}^{\xi}\left[(N-1) H(0, y) u_{\xi \xi}^{*}\left(s+s_{0}\right)+\tilde{f}_{u}^{1} u_{\xi}^{*}\left(s+s_{0}\right)\right] u_{\xi}^{*}\left(s+s_{0}\right) d s d \xi \\
& =\int_{ \pm \infty}^{0}\left[u_{\xi \xi}^{1}(\xi, y) u_{\xi}^{*}\left(\xi+s_{0}\right)-u_{\xi}^{1}(\xi, y) u_{\xi \xi}^{*}\left(\xi+s_{0}\right)\right] d \xi \\
& =u_{\xi}^{ \pm, 1}(0, y) u_{\xi}^{*}\left(s_{0}\right)-2 I_{3}^{ \pm} .
\end{aligned}
$$


Appendix E. (proof of Lemma 4.3). (i) We study the operator $\Pi_{-}+\Pi_{+}$ instead of $\mathcal{T}$. The problems (1.11) and (1.10) are rewritten as

$$
\begin{cases}Z_{r r}^{+}+\frac{N-1}{r} Z_{r}^{+}+\frac{1}{r^{2}} \Delta^{S} Z^{+}=0, & r_{0}<r<R, \\ Z^{+}\left(r_{0}, y\right)=q, \quad Z_{r}^{+}(R, y)=0, & y \in S,\end{cases}
$$

$$
\begin{cases}Z_{r r}^{-}+\frac{N-1}{r} Z_{r}^{-}+\frac{1}{r^{2}} \Delta^{S} Z^{-}=0, & 0<r<r_{0} \\ Z_{r}^{-}(0, y)=0, \quad Z^{-}\left(r_{0}, y\right)=q, & y \in S,\end{cases}
$$

where $\Delta^{S}$ is the Laplacian on $S=S^{N-1}$. Note that $\Pi_{ \pm}$have the same complete system of eigenfunctions as that of $\Delta^{S}$.

Let $\left\{\ell_{j}, \beta_{j}^{m}(y)\right\}_{j=1}^{\infty}$ be the complete system of an eigenpair for $-\Delta^{S}$, where $\ell_{j}=$ $j(j+N-2), j=1,2, \ldots$ If we take $q=\beta_{j}$, then the solutions of $(E .1)_{ \pm}$separate as $Z^{ \pm}(r, y)=R^{ \pm, j}(r) \beta_{j}(y)$. The equations for $R^{ \pm, j}(r)$ are, respectively,

$$
\begin{gathered}
\begin{cases}R_{r r}^{+, j}+\frac{N-1}{r} R_{r}^{+, j}-\frac{l_{j}}{r^{2}} R^{+, j}=0, & r_{0}<r<R, \\
R^{+, j}\left(r_{0}\right)=1, \quad R_{r}^{+, j}(R)=0, & y \in S,\end{cases} \\
\begin{cases}R_{r r}^{-, j}+\frac{N-1}{r} R_{r}^{-, j}-\frac{l_{j}}{r^{2}} R^{-, j}=0, & 0<r<r_{0}, \\
R_{r}^{-, j}(0)=0, \quad R^{-, j}\left(r_{0}\right)=1, & y \in S .\end{cases}
\end{gathered}
$$

In terms of the solutions $R^{ \pm, j}$, the operator $\Pi_{-}+\Pi_{+}$acting on $\beta_{j}$ are expressed as

$$
\left(\Pi_{-}+\Pi_{+}\right) \beta_{j}=\left(R_{r}^{-, j}\left(r_{0}\right)-R_{r}^{+, j}\left(r_{0}\right)\right) \cdot \beta_{j} .
$$

By using the fundamental solutions $r^{j}$ and $r^{-(j+N-2)}$, we have

$$
R^{-, j}(r)=\frac{1}{r_{0}^{j}} r^{j}, \quad R^{+, j}(r)=A^{+, j} r^{j}+B^{+, j} r^{-(j+N-2)},
$$

where

$$
\begin{aligned}
& A^{+, j}=\frac{(j+N-2) R^{-(j+N-1)}}{\Delta}, \quad B^{+, j}=\frac{j R^{j-1}}{\Delta}, \\
& \Delta=(j+N-2) r_{0}^{j} R^{-(j+N-1)}+j r_{0}^{-(j+N-2)} R^{j-1}
\end{aligned}
$$

Then, we have

$$
R_{r}^{-, j}\left(r_{0}\right)-R_{r}^{+, j}\left(r_{0}\right)=\frac{j(2 j+N-2)}{\Delta r_{0}^{j+N-1} R^{-(j-1)}}
$$

for $j \geq 1$. Therefore, the eigenvalue of $\mathcal{T}$ is given by (4.3) and (4.4) since it is defined by the inverse of the right-hand side of (E.2).

(ii) This can be shown by simple computation so we omit the proof. 
Appendix F. (proof of Lemma 4.7). (i) We study the operator $\Pi_{-}+\Pi_{+}$ instead of $\mathcal{T}$. The problems (1.11) and (1.10) are recast as

$$
\begin{aligned}
& (\text { F.1 })_{+} \begin{cases}Z_{x x}^{+}+Z_{y y}^{+}=0, & (x, y) \in\left(x_{0}, X\right) \times(0, Y), \\
Z^{+}\left(x_{0}, y\right)=q, \quad Z_{r}^{+}(X, y)=0, & y \in(0, Y),\end{cases} \\
& \left(\begin{array}{ll}
Z_{x x}^{-}+Z_{y y}^{-}=0, \\
Z_{r}^{-}(0, y)=0, \quad Z^{-}\left(x_{0}, y\right)=q, & y \in(0, Y),
\end{array}\right.
\end{aligned}
$$

Note that $\Pi_{ \pm}$have the same complete system of eigenfunctions as that of $-\frac{d^{2}}{d y^{2}}$ under the Neumann boundary condition.

Let $\left\{\tau_{j}^{2}, \beta_{j}(y)\right\}_{j=1}^{\infty}$ be the complete system of an eigenpair for $-\frac{d^{2}}{d y^{2}}$, where

$$
\beta_{j}(y)=\cos \left(\tau_{j} y\right), \quad \tau_{j}:=\frac{\pi j}{Y} .
$$

If we take $q=\beta_{j}$, then the solutions of $(F \cdot 1)_{ \pm}$separate as $Z^{ \pm}(x, y)=\zeta^{ \pm, j}(x) \beta_{j}(y)$. The equations for $\zeta^{ \pm, j}(x)$ are, respectively,

$$
\begin{gathered}
\begin{cases}\zeta_{x x}^{+, j}-\tau_{j}^{2} \zeta^{+, j}=0, & x_{0}<x<X, \\
\zeta^{+, j}\left(x_{0}\right)=1, & \zeta_{x}^{+, j}(X)=0,\end{cases} \\
\begin{cases}\zeta_{x x}^{-, j}-\tau_{j}^{2} \zeta^{-, j}=0, & 0<x<x_{0}, \\
\zeta_{x}^{-, j}(0)=0, & \zeta^{-, j}\left(x_{0}\right)=1 .\end{cases}
\end{gathered}
$$

In terms of the solutions $\zeta^{ \pm, j}$, the operator $\Pi_{-}+\Pi_{+}$acting on $\beta_{j}$ are expressed as,

$$
\left(\Pi_{-}+\Pi_{+}\right) \beta_{j}=\left(\zeta_{x}^{-, j}\left(x_{0}\right)-\zeta_{x}^{+, j}\left(x_{0}\right)\right) \cdot \beta_{j} .
$$

By using the fundamental solutions $e^{\tau_{j} x}$ and $e^{-\tau_{j} x}$, we have

$$
\zeta^{ \pm, j}(x)=c_{1}^{ \pm} e^{\tau_{j} x}+c_{2}^{ \pm} e^{-\tau_{j} x},
$$

where

$$
\begin{gathered}
{\left[\begin{array}{l}
c_{1}^{-} \\
c_{2}^{-}
\end{array}\right]=\frac{1}{\Delta^{-}}\left[\begin{array}{l}
1 \\
1
\end{array}\right], \quad\left[\begin{array}{l}
c_{1}^{+} \\
c_{2}^{+}
\end{array}\right]=\frac{1}{\Delta^{+}}\left[\begin{array}{l}
e^{-\tau_{j} X} \\
e^{\tau_{j} X}
\end{array}\right],} \\
\Delta^{-}:=e^{x_{0} \tau_{j}}+e^{-x_{0} \tau_{j}}, \quad \Delta^{+}:=e^{\left(X-x_{0}\right) \tau_{j}}+e^{-\left(X-x_{0}\right) \tau_{j}} .
\end{gathered}
$$

Then, we have

$$
\zeta_{x}^{-, j}\left(x_{0}\right)-\zeta_{x}^{+, j}\left(x_{0}\right)=\frac{2 \tau_{j} \sinh X \tau_{j}}{\cosh X \tau_{j}+\cosh \bar{u} X \tau_{j}}
$$

for $j \geq 1$. Therefore, the eigenvalue of $\mathcal{T}$ is given by (4.9) and (4.10) since it is defined by the inverse of the right-hand side of (F.2).

(ii) This can be shown by simple computation so we omit the proof. 
Appendix G. (proof of Lemma 5.1). Without loss of generality, we can normalize $\theta_{c}$ as $\theta_{c}=1$. Multiplying $\bar{z}$ (complex conjugate of $z$ ) to the second equation of (5.4) and integrating by parts, we obtain

$$
-D \int_{\Omega}|\nabla z|^{2} d x+\int_{\Omega} g_{u} w \bar{z} d x+\epsilon \int_{\Omega} g_{v}|z|^{2} d x=\epsilon^{2} \lambda_{1}(\epsilon) \int_{\Omega}|z|^{2} d x
$$

where

$$
|\nabla z|^{2}:=\sum_{i=1}^{N} \frac{\partial z}{\partial x^{i}} \frac{\partial \bar{z}}{\partial x^{i}}, \quad|z|^{2}:=z \bar{z} .
$$

Noting that $w^{ \pm, i}(\xi, y)(\mathrm{i}=0,1)$ decays exponentially as $|\xi| \rightarrow \infty$, we have

$$
\begin{aligned}
\int_{\Omega} g_{u} w \bar{z} d x= & \int_{\Omega_{+}\left(\Gamma^{*}\right)} g_{u} w^{+} \overline{z^{+}} d x+\int_{\Omega_{-}\left(\Gamma^{*}\right)} g_{u} w^{-} \overline{z^{-}} d x \\
= & g_{u}^{*,+} \int_{\Omega_{+}\left(\Gamma^{*}\right)}\left[\epsilon h_{v}^{+}\left(v^{*}\right) q_{c}+w^{0}(r, y) \omega(r)\right] \overline{q_{c}} d x \\
& +g_{u}^{*,-} \int_{\Omega_{-}\left(\Gamma^{*}\right)}\left[\epsilon h_{v}^{-}\left(v^{*}\right) q_{c}+w^{0}(r, y) \omega(r)\right] \overline{q_{c}} d x+O\left(\epsilon^{2}\right) \\
= & \epsilon\left[g_{u}^{*,+} h_{v}^{+}\left(v^{*}\right)\left|\Omega_{+}\left(\Gamma^{*}\right)\right|+g_{u}^{*,-} h_{v}^{-}\left(v^{*}\right)\left|\Omega_{-}\left(\Gamma^{*}\right)\right|\right]\left|q_{c}\right|^{2} \\
& +\epsilon\left[C^{+}(\epsilon)+C^{-}(\epsilon)\right] \overline{q_{c}}+O\left(\epsilon^{2}\right),
\end{aligned}
$$

where $\left|\Omega_{ \pm}\left(\Gamma^{*}\right)\right|$ is volume of domain $\Omega_{ \pm}\left(\Gamma^{*}\right)$,

$$
C^{ \pm}(\epsilon):=\frac{g_{u}^{*, \pm}}{\epsilon} \int_{\Omega_{ \pm}\left(\Gamma^{*}\right)} w^{0}(r / \epsilon, y) \omega(r) d x=\frac{g_{u}^{*, \pm}}{\epsilon} \int_{\Omega_{ \pm}\left(\Gamma^{*}\right)} u_{\xi}^{*}(r / \epsilon) \omega(r) d x>0
$$

for small $\epsilon>0$ and $g_{u}^{*, \pm}=g_{u}\left(h^{ \pm}\left(v^{*}\right), v^{*}\right)$. Using the fact that

$$
\sup _{x \in \Omega}|\nabla z|=O(\epsilon) \text { and } \int_{\Omega}|z|^{2} d x=O(1) \quad \text { as } \epsilon \downarrow 0,
$$

and dividing (G.1) by $\epsilon$, we have

$$
\begin{aligned}
\left(C^{+}(\epsilon)+C^{-}(\epsilon)\right) \overline{q_{c}}= & -\left(g_{u}^{*,+} h_{v}^{+}\left(v^{*}\right)\left|\Omega_{+}\left(\Gamma^{*}\right)\right|+g_{u}^{*,-} h_{v}^{-}\left(v^{*}\right)\left|\Omega_{-}\left(\Gamma^{*}\right)\right|\right)\left|q_{c}\right|^{2} \\
& +\left(g_{u}^{*,+}\left|\Omega_{+}\left(\Gamma^{*}\right)\right|+g_{u}^{*,-}\left|\Omega_{-}\left(\Gamma^{*}\right)\right|\right)\left|q_{c}\right|^{2}+O(\epsilon) .
\end{aligned}
$$

This implies Re $q_{c}<0$ since $g_{u}^{*, \pm}>0, g_{v}^{*, \pm}=g_{v}\left(h^{ \pm}\left(v^{*}\right), v^{*}\right)<0$, and $h_{v}^{ \pm}\left(v^{*}\right)=$ $1 / f_{u}\left(h^{ \pm}\left(v^{*}\right), v^{*}\right)<0$. Then we conclude that

$$
\operatorname{Re} \lambda_{1}^{*}=\operatorname{Re} \frac{J^{\prime}\left(v^{*}\right) q_{c}}{m^{2}}<0 .
$$

Acknowledgment. The authors thank the anonymous referees for many valuable comments and pointing out an important reference. 


\section{REFERENCES}

[1] M. Bahiana and Y. Oono, Cell dynamical system approach to block copolymers, Phys. Rev., 41 (1990), pp. 6763-6771.

[2] R. Choksi, Scaling laws in microphase separation of diblock copolymers, J. Nonlinear Sci., 11 (2001), pp. 223-236.

[3] R. Choksi And X. Ren, On the derivation of a density functional theory for microphase separation of diblock copolymers, J. Statist. Phys., 113 (2003), pp. 151-176.

[4] A. Doelman and H. van der Ploeg, Homoclinic stripe patterns, SIAM J. Appl. Dyn. Syst., 1 (2002), pp. 65-104.

[5] M. DeL PINo, Radially symmetric internal layers in a semilinear elliptic system, Trans. Amer. Math. Soc., 347 (1995), pp. 4807-4837.

[6] H. Hasegawa, H. Tanaka, K. Yamasaki, and T. Hashimoto, Macromolecules, 20 (1987), pp. 1651.

[7] T. Hashimoto, M. Shibayama, and H. Kawai, Macromolecules, 16 (1983), pp. 1093.

[8] T. Hashimoto, H. Tanaka, and H. Hasegawa, Molecular Conformation and Dynamics of Macromolecules in Condensed Systems, M. Nagasawa, ed., Elsevier, Amsterdam, 1998.

[9] H. IKEDA, On the asymptotic solutions for a weakly coupled elliptic boundary value problem with a small parameter, Hiroshima Math. J., 16 (1986), pp. 227-250.

[10] Y. Nishiura, Coexistence of infinitely many stable solutions to reaction diffusion systems in the singular limit, in Dynamics Reported (New Series) Vol. 3, Springer-Verlag, Berlin, 1994, pp. 25-103.

[11] Y. NishiURA AND H. FuJII, Stability of singularly perturbed solutions to systems of reactiondiffusion equations, SIAM J. Math. Anal., 18 (1987), pp. 1726-1770.

[12] Y. Nishiura And I. Ohnishi, Some aspect mathematical of the micro-phase separation in diblock copolymers, Phys. D, 84 (1995), pp. 31-39.

[13] Y. Nishiura AND H. Suzuki, Nonexistence of higher dimensional stable Turing patterns in the singular limit, SIAM J. Math. Anal., 29 (1998), pp. 1087-1105.

[14] I. Ohnishi, Y. Nishiura, M. Imai, and Y. Matsushita, Analytical solutions describing the phase separation driven by a free energy functional containing a long-range interaction term, Chaos, 9 (1999), pp. 329-341.

[15] Y. OsHITA, On stable nonconstant stationary solutions and mesoscopic patterns for FitzHughNagumo equations in higher dimensions, J. Differential Equations, 188 (2003), pp. 110-134.

[16] T. OHTA AND K. KaWASAKI, Equilibrium morphology of block copolymer melts, Macromolecules, 19 (1986), pp. 2621

[17] K. Sakamoto, Internal layers in high-dimensional domains, Proc. Roy. Soc. Edinb., 128 (1998), pp. 359-401.

[18] K. Sakamoto, Spatial homogenization and internal layers in a reaction-diffusion system, Hiroshima Math. J., 30 (2000), pp. 377-402.

[19] K. Sakamoto And H. Suzuki, Spherically symmetric internal layers for activator-inhibitor systems: I. Existence by a Lyapunov-Schmidt reduction, J. Differential Equations, to appear.

[20] K. Sakamoto and H. Suzuki, Spherically symmetric internal layers for activator-inhibitor systems: II. Stability and symmetry breaking bifurcations, J. Differential Equations, to appear.

[21] H. SuZukI, Asymptotic characterization for interfacial patterns for reaction diffusion systems, Hokkaido Math. J., 26 (1997), pp. 631-667.

[22] M. TANiguchi AND Y. Nishiura, Instability of planar interfaces in reaction-diffusion systems, SIAM J. Math. Anal., 25 (1994), pp. 99-134.

[23] M. TANiguChI AND Y. NishiuRA, Stability and characteristic wavelength of planar interfaces in the large diffusion limit of the inhibitor, Proc. Roy. Soc. Edinburgh Sect. A, 126 (1996), pp. $117-145$.

[24] T. Teramoto and Y. Nishiura, Double gyroid morphology in a gradient system with nonlocal effects, J. Phys. Soc. Japan, 71 (2002), pp. 1611-1614.

[25] X. Ren AND J. WeI, On the multiplicity of solutions of two nonlocal variational problems, SIAM J. Math. Anal., 31 (2000), pp. 909-924.

[26] X. Ren And J. WeI, Concentrically layered energy equilibria of the di-block copolymer problem, European J. Appl. Math., 13 (2002), pp. 479-496.

[27] X. Ren AND J. WeI, On the spectra of three-dimensional Lamellar solutions of the Diblock copolymer problem, SIAM J. Math. Anal., 35 (2003), pp. 1-32. 